\title{
COCYCLE TWISTS AND EXTENSIONS OF BRAIDED DOUBLES
}

\author{
YURI BAZLOV AND ARKADY BERENSTEIN
}

\begin{abstract}
It is well known that central extensions of a group $G$ correspond to 2-cocycles on $G$. Cocycles can be used to construct extensions of $G$-graded algebras via a version of the Drinfeld twist introduced by Majid. We show how 2-cocycles can be defined for an abstract monoidal category $\mathcal{C}$, following Panaite, Staic and Van Oystaeyen. A braiding on $\mathcal{C}$ leads to analogues of Nichols algebras in $\mathcal{C}$, and we explain how the recent work on twists of Nichols algebras by Andruskiewitsch, Fantino, Garcia and Vendramin fits in this context.

Furthermore, we propose an approach to twisting the multiplication in braided doubles, which are a class of algebras with triangular decomposition over $G$. Braided doubles are not $G$-graded, but may be embedded in a double of a Nichols algebra, where a twist may be carried out if careful choices are made. This is a source of new algebras with triangular decomposition. As an example, we show how to twist the rational Cherednik algebra of the symmetric group by the cocycle arising from the Schur covering group, obtaining the spin Cherednik algebra introduced by Wang.
\end{abstract}

\section{CONTENTS}

0. Introduction

1. Cocycles, central extensions, the Schur multiplier, twisted group algebras

2. The Schur multiplier of $S_{n}$

3. Cocycle twists and cocycle extensions of group-graded algebras

4. Cocycle twists and the lazy cohomology of a monoidal category

5. Cocycle twists of braidings and Nichols algebras

6. Extensions and twists of Yetter-Drinfeld modules

7. Braided doubles

8. Braided Heisenberg doubles and braided Weyl algebras

9. Extensions and twists of other braided doubles

10. Covering Cherednik algebras and spin Cherednik algebras

2010 Mathematics Subject Classification. Primary 16G99; Secondary 16T05, 16T25.

We acknowledge the support of the LMS Research in Pairs grant ref. 41024. The second named author was partially supported by the NSF grant DMS-1101507. 


\section{INTRODUCTION}

0.1. Background. Cohomology and homology groups arise in diverse areas of modern mathematics. It seems fair to say that homological algebra owes its current level of abstraction to category theory, which arose from papers by Eilenberg and Mac Lane in 1940s, for example [22]. However, certain specific cohomology groups were studied by algebraists much earlier. A notable example is the Schur multiplier $M(G)$ of a finite group $G$, introduced by Schur in 1904-07 in his work [52, 53. on projective representations. Modern understanding of the finite abelian group $M(G)$ as the second cohomology group $H^{2}\left(G, \mathbb{C}^{\times}\right)$came from papers such as Green 31] in mid-1950s. This led to a number of generalisations and new interpretations of this important group, see for example the theoretical physics paper [57] by Vafa and Witten.

In Schur's approach, what is now known as a 2-cocycle on $G$ appears in the guise of a factor set of a projective representation of $G$. In general, factor sets correspond to central extensions of $G$. The equation defining a factor set - and a 2-cocycle — is simply a manifestation of the associative law of multiplication.

Because of this latter point, 2-cocycles can be used to construct central extensions and twists of associative algebras, not only groups. One may recall deformations of an algebra $A$ over a field $k$ in the sense of Gerstenhaber 28; they are algebras over a complete local ring with field of residues $k$, and are governed by Hochschild cocycles on $A$. On the other hand, when $A$ is a $G$-graded algebra, twists of $A$ by 2 -cocycles on $G$ with values in $k^{\times}$can be constructed.

In the present paper, cocycle twists of associative algebras are the main object. We pursue two principal goals:

- to explain what are the Schur multiplier and cocycle twists in the most general setting of a monoidal category, and to apply cocycle twists to Nichols-type algebras if the category is braided;

- to construct cocycle twists of braided doubles (for which the above general approach is not sufficient), thus obtaining new algebras with triangular decomposition.

The rest of this Introduction serves to explain what is achieved with respect to the above two goals. We outline the structure of the paper and give some illustrative examples.

0.2. A brief overview. We begin by recalling the necessary background on cocycles and the Schur multiplier of a finite group $G$, and study the Schur multiplier of $S_{n}$ in some detail. We explain how to twist a $G$-graded algebra by a cocycle. To work with algebras in a more general setting, for example algebras over commutative rings, we invoke the definition of an abstract monoidal category $\mathcal{C}$ of which $G$-graded vector spaces are an example. It turns out that the Schur multiplier of $G$ can be defined more generally purely in terms of the monoidal structure on $\mathcal{C}$, leading to a group $H_{\ell}^{2}(\mathcal{C})$. 
In the context of Drinfeld's approach (made explicit by Majid) to twisting the monoidal product $\otimes$, the group $H_{\ell}^{2}(\mathcal{C})$ represents only those twists that leave $\otimes$ unchanged; it is thus a vertex group in the groupoid of more general twists.

A braiding on a monoidal category $\mathcal{C}$ leads to an analogue of Nichols algebras in $\mathcal{C}$, and we show that this class of algebras is closed under cocycle twisting. Based on our earlier work, we explain the link between Nichols algebras and braided doubles, a class of algebras with triangular decomposition. This suggests an approach to cocycle twisting of braided doubles. However, unlike the functorial construction of a twist on a Nichols algebra, twisting a braided double depends on carefully made choices, and is not guaranteed to work in general.

We show how to make the necessary choices for the braided double known as the rational Cherednik algebra of $S_{n}$, due to Etingof and Ginzburg. This sees the Schur covering group $T_{n}$ of $S_{n}$ play the role of a reflection group, acting on a free $\mathbb{C}[z] /<z^{2}-1>$-module of rank $n$ rather than a vector space, with the root system

$$
\left\{ \pm\left(e_{i}-z e_{j}\right) \mid 1 \leq i, j \leq n, i \neq j\right\}
$$

The twisted version of the rational Cherednik algebra is isomorphic to an algebra earlier obtained by Wang. We leave the more general case of a complex reflection group to our upcoming paper [8].

The paper contains a certain amount of survey material on topics related to cocycle twisting, Nichols algebras and algebras with triangular decomposition. This is to help to introduce the reader to these topics and to put our results in context.

0.3. Cocycles. In Sections 13, we review the construction of the second cohomology group $H^{2}(G, \Gamma)$. Recall that the group $Z^{2}(G, \Gamma)$ of normalised 2-cocycles consists of solutions $\mu: G \times G \rightarrow \Gamma$ to the functional equations

$$
\mu(g, h) \mu(g h, k)=\mu(g, h k) \mu(h, k), \quad \mu(1, g)=\mu(g, 1)=1
$$

where $g, h, k \in G$. Here $\Gamma$ is an abelian group, so that the pointwise multiplication in $Z^{2}(G, \Gamma)$ is automatically commutative. The group $H^{2}(G, \Gamma)$ is the quotient of $Z^{2}(G, \Gamma)$ modulo a suitably defined subgroup of coboundaries. The central extension of $G$ by $\Gamma$ corresponding to $\mu \in Z^{2}(G, \Gamma)$ is constructed in two steps: first, consider the trivial extension $\Gamma \times G$; then introduce a new, $\mu$-twisted, group multiplication on $\Gamma \times G$.

We would like to construct extensions of algebras, so our main case is $\Gamma=R^{\times}$, the multiplicative group of a commutative ring $R$. A cocycle $\mu \in Z^{2}\left(G, R^{\times}\right)$can be used to twist the multiplication in a $G$-graded associative algebra $(A, \cdot)$ over $R$, obtaining a new associative algebra $A_{\mu}=\left(A, \star_{\mu}\right)$ where

$$
a \star_{\mu} b=\mu(g, h) a b, \quad \text { if } a \in A_{g}, b \in A_{h} .
$$


If $R$ is itself an algebra over another commutative ring, say $\mathbb{C}$, then a $G$-graded $\mathbb{C}$-algebra $A$ can be trivially extended to an $R \otimes_{\mathbb{C}} A$, then twisted by $\mu$. We denote the result by $\widetilde{A}_{\mu}$ and refer to it as the cocycle extension of $A$ by $\mu$.

We note that the cocycle twist construction works for algebras that have $G$-grading. Of course, the group algebra of $G$ (or of any group $H$ equipped with a group homomorphism $H \rightarrow G$ ) has $G$-grading. We will now look at two other classes of natural examples of $G$-graded algebras.

0.4. Example: semidirect products, central simple algebras, relative Brauer group. If $A$ is an $R$-algebra with a covariant action of $G$, one can form the semidirect product algebra

$$
A \rtimes G,
$$

see 7.1. This has a natural $G$-grading where $a g(a \in A, g \in G)$ has degree $g$. The algebra $A \rtimes G$ can therefore be twisted by a cocycle $\mu \in Z^{2}\left(G, R^{\times}\right)$, giving rise to a new algebra $(A \rtimes G)_{\mu}$.

The following important example of twisted $A \rtimes G$ arose in 1930s in the study of central simple algebras over a field. Let $K / F$ be a finite Galois extension of fields with Galois group $G$. Each $\mu \in Z^{2}\left(G, K^{\times}\right)$gives rise to the $F$-algebra

$$
(K \rtimes G)_{\mu},
$$

which turns out to be a central simple $F$-algebra split by $K$. Moreover, the map which sends $\mu \in Z^{2}\left(G, K^{\times}\right)$to the class of $(K \rtimes G)_{\mu}$ in the relative Brauer group $\operatorname{Br}(K / F)$ is a group isomorphism between $H^{2}\left(G, K^{\times}\right)$and $B r(K / F)$. In particular, the algebra $K \rtimes G$ (where $\mu=1$ is the trivial cocycle) is isomorphic to the matrix algebra $M_{n}(F)$ (which represents the trivial class in the Brauer group), with $n=|G|$. Central simple $F$-algebras of the form $(K \rtimes G)_{\mu}$ are called crossed products. Their story is now classical, with details given in algebra textbooks such as [21, ch. 17]. They are a good illustration of the cocycle twist technique for semidirect products, but we will not consider them in the present paper.

0.5. Example: Nichols algebras over $G$. Nichols algebras over $G$ are a class of $G$-graded algebras, which are quite different from the semidirect products in the previous example, and are of a much more recent origin. Nichols algebras play an important role in the present paper. To define a Nichols algebra, one needs a Yetter-Drinfeld module $Y$ over $\mathbb{C} G$, which is a $G$-graded space with a compatible $G$-action, see 6.1. The grading and the action give rise to a braiding on $Y$, i.e., an invertible linear map $\Psi: Y \otimes Y \rightarrow Y \otimes Y$ which solves the braid equation

$$
\left(\Psi \otimes \operatorname{id}_{Y}\right)\left(\operatorname{id}_{Y} \otimes \Psi\right)\left(\Psi \otimes \operatorname{id}_{Y}\right)=\left(\operatorname{id}_{Y} \otimes \Psi\right)\left(\Psi \otimes \operatorname{id}_{Y}\right)\left(\operatorname{id}_{Y} \otimes \Psi\right)
$$

(both sides are endomorphisms of $Y^{\otimes 3}$ ). The Nichols algebra of $Y$ is defined as

$$
\mathcal{B}(Y)=T(Y) / \operatorname{ker} I_{\Psi}
$$


meaning that it is generated by $Y$ as an algebra, with relations determined in a certain way by $\Psi$. In fact, $I_{\Psi} \subset T(Y)$ is the kernel of the braided symmetriser attached to $\Psi$, originally due to Woronowicz 61; and $\mathcal{B}(Y)$ is a graded $G$-graded algebra.

Following a suggestion of Kaplansky, Nichols algebras were so named by Andruskiewitsch and Schneider in [2] because their precursor arose in the work of Nichols [46]. Andruskiewitsch and Schneider pioneered the use of Nichols algebras in classifying pointed Hopf algebras; their comprehensive survey [3] accurately reflects the state of the art at the time. A key property of Nichols algebras - that they are braided Hopf algebras — was not addressed in [46] as the formalism of Hopf algebras in braided categories was yet to be introduced. Nichols algebras as braided Hopf algebras appeared in Majid [39] where they arise naturally in the context of a duality pairing in a braided monoidal category.

Note that the relations in the Nichols algebra $\mathcal{B}(Y)$ are given implicitly. Hence, finding the Hilbert series of $\mathcal{B}(Y)$ or even determining whether $\operatorname{dim} \mathcal{B}(Y)<\infty$ can be highly non-trivial (and important in Hopf algebra classification problems). In fact, in one of the key examples of a Nichols algebra for $G=S_{n}$, considered below, these questions are still open for $n \geq 6$. Classification of finite-dimensional Nichols algebras over a finite group $G$ is a major direction of research into Nichols algebras.

It turns out that cocycle twists of Nichols algebras over $G$ are again Nichols algebras. For cocycles with values in the base field, this follows for example from a result of Majid and Oeckl 44]. Cocycle twists are thus a useful tool in studying Nichols algebras, because they produce new Nichols algebras with the same Hilbert series. In the present paper we perform a cocycle twist of a Nichols algebra in a more general context of a braided monoidal category.

0.6. The lazy cohomology of a monoidal category. In Section 4, we deal with a far-reaching generalisation of the Schur multiplier $M(G)$ of a finite group $G$ - the lazy cohomology of an abstract monoidal category $(\mathcal{C}, \otimes)$, denoted $H_{\ell}^{2}(\mathcal{C}, \otimes)$. This is a group which is not necessarily abelian. The $M(G)=H^{2}\left(G, \mathbb{C}^{\times}\right)$becomes a particular case of a lazy cohomology group by considering $\mathcal{C}=\mathcal{M}^{G}$, the category of $G$-graded $\mathbb{C}$-vector spaces, with a standard tensor product $\otimes=\otimes_{\mathbb{C}}$.

If the category $(\mathcal{C}, \otimes)$ is linear over a field $k$, then it (subject to some technical assumptions) embeds in a category of comodules of a Hopf algebra $H$ over $k$. This is the subject of the Lyubashenko-Majid reconstruction theory, see [41, chapter 9]. In particular, the group algebra $\mathbb{C} G$ is reconstructed from the monoidal category $\mathcal{M}^{G}$. For a Hopf algebra $H$ over $k$, the lazy cohomology was introduced by Bichon and Carnovale [10 based on a work [51] by Schauenburg. Even more generally, for arbitrary monoidal categories the lazy cohomology is defined in terms of laycles (lazy cocycles) by Panaite, Staic and Van Oystaeyen [47. Such general approach allows one to work, 
for example, with a category of $G$-graded $R$-modules where $R$ is a commutative ring. Among other possible areas of application, let us point out the monoidal category of sets as used, for instance, in Gateva-Ivanova - Majid [27.

0.7. The group of lazy cocycles vs. the groupoid of Drinfeld twists. The categorical construction of cocycles and twists should be compared to the Drinfeld twists, introduced by Majid in [41, section 2.3] for a module category of a Hopf algebra $H$ over $k$. If $\Delta: H \rightarrow H \otimes H$ denotes the coproduct on $H$, then Drinfeld twists essentially are invertible elements $F \in H \otimes H$ such that $\Delta_{F}(h):=F(\Delta h) F^{-1}$ is again a coproduct on the same algebra $H$. This condition leads to an equation on $F$ which is weaker than the quantum Yang-Baxter equation.

One should note that Drinfeld twists in general do not form a group under multiplication. Indeed, in [41, 2.3] Majid defines the second non-abelian cohomology of a Hopf algebra as a set not a group. We observe, however, that Drinfeld twists form a groupoid. The vertices of the groupoid are all possible coproducts $\Delta$ on a given algebra $H$. The arrows between two coproducts $\Delta$ and $\Delta^{\prime}$ are all the Drinfeld twists $F \in H \otimes H$ such that $\Delta^{\prime}=\Delta_{F}$.

In this picture, the lazy cocycles of the module category of the Hopf algebra $H$ (with fixed coproduct $\Delta$ ) are precisely the Drinfeld twists $F \in H \otimes H$ such that $\Delta_{F}=\Delta$. (These twists commute with $\Delta h$ for all $h \in H$ and are sometimes called invariant twists, see Guillot - Kassel [33.) Thus, the group of lazy cocycles in $H \otimes H$ is a vertex group of the groupoid of Drinfeld twists.

This picture should generalise to abstract categories, where to a category $\mathcal{C}$ there should be associated a groupoid whose vertices are monoidal products $\otimes$ on $\mathcal{C}$, and arrows are natural isomorphisms $\otimes \rightarrow \otimes^{\prime}$ that satisfy the abstract Drinfeld twist constraint.

Although we do not consider non-invariant Drinfeld twists in the present paper, we would like to say a few words about them in this Introduction. Drinfeld twists are used in the quantum group theory and modern theoretical physics to deform the associative product on a group-graded algebra, more generally a module algebra $A$ of a Hopf algebra $H$. If $F \in H \otimes H$ is a twist, the action of $F^{-1}$ on $A \otimes A$ intertwines the original product - on $A$ with a new associative product $\star=\star_{F}$. If $G$ is a finite group, a $G$-graded algebra is the same as an algebra with a covariant action of the commutative Hopf algebra $\mathbb{C}(G)=\operatorname{Fun}(G, \mathbb{C})$. The twist equation and invertibility for $\mu \in \mathbb{C}(G) \otimes \mathbb{C}(G) \cong \mathbb{C}(G \times G)$ mean precisely that $\mu$ is a 2-cocycle with values in $\mathbb{C}^{\times}$.

The Drinfeld twist expresses deformation quantisation in a Hopf algebraic language, and is a powerful formalism for introducing noncommutativity. See the survey physics paper [4] where the Moyal quantisation is explained in terms of a Drinfeld twist, and [12] where the twistor theory and the Penrose-Ward transform are quantised using Drinfeld twists. 
0.8. Braided categories. Cocycle twists of braidings. Nichols algebras. In Section [5, we work with a monoidal category with a braiding, which is a type of a commutativity constraint. Braidings on monoidal categories arise naturally in the theory of quantum groups. In the reconstruction picture where monoidal categories are thought of as (subcategories of) categories of modules over a Hopf algebra $H$, see 0.6 , braidings correspond to quasitriangular structures on Hopf algebras, which essentially are universal solutions to the quantum Yang-Baxter equation. It turns out that cocycles on a monoidal category $\mathcal{C}$ can be used to twist braidings on $\mathcal{C}$ : the group $Z^{2}(\mathcal{C})$ of cocycles acts on the class of braidings.

It is in Section 5 that we introduce the categorical analogue of Nichols algebras, discussed above in 0.5 If $\Psi$ is a braiding on a monoidal category $\mathcal{C}$, then, subject to some restrictions, to each object of $V$ of $\mathcal{C}$ there is associated an algebra $\mathcal{B}(V, \Psi)$ in $\mathcal{C}$. The Nichols algebras over a group $G$ are a particular case of this construction where $\mathcal{C}={ }_{G} \mathcal{Y} \mathcal{D}^{G}$ is the category of Yetter-Drinfeld modules over $G$. However, our technique of working with general monoidal categories allows us to consider Nichols algebras over a commutative ring $R$, something which has not been done so far in the Hopf algebra literature.

0.9. Yetter-Drinfeld modules and their twists. Main examples for $S_{n}$. In Section 6 we explain the construction of the category of Yetter-Drinfeld modules. This is a braided monoidal category, and it can be said that raison d'être of Yetter-Drinfeld modules is that that they provide an example of such a category, extremely useful in the quantum group theory. It is a result of Majid that the category ${ }_{H} \mathcal{Y} \mathcal{D}^{H}$ of Yetter-Drinfeld modules over a Hopf algebra $H$, finite-dimensional over a field $k$, is equivalent to the category of modules over the Drinfeld double $D(H)$ of $H$, which is a standard example of a quasitriangular Hopf algebra.

In the present paper we only consider Yetter-Drinfeld modules over a group algebra of $G$. We do not assume $k$-linearity, working in a slightly more general situation of free $R$-modules where $R$ is a commutative ring; this leads to the braided monoidal category $R_{R, G} \mathcal{Y D}^{G}$. It turns out that every cocycle $\mu \in Z^{2}\left(G, R^{\times}\right)$can be viewed as a lazy cocycle in $Z^{2}\left({ }_{R, G} \mathcal{Y} \mathcal{D}^{G}\right)$. So, unlike in the case of an abstract monoidal category, twists can be made quite explicit in ${ }_{R, G} \mathcal{Y} \mathcal{D}^{G}$. And it is for Yetter-Drinfeld modules that the result, mentioned above in 0.5. holds: if the Nichols-type algebra $\mathcal{B}(Y)$, where $Y \in \operatorname{Ob}\left({ }_{R, G} \mathcal{Y} \mathcal{D}^{G}\right)$, is twisted by $\mu$, the result is $\mathcal{B}\left(Y_{\mu}\right)$ where $Y_{\mu} \in \operatorname{Ob}\left({ }_{R, G} \mathcal{Y} \mathcal{D}^{G}\right)$ is a certain other Yetter-Drinfeld module called the twist of $Y$ by $\mu$. In the $k$-linear situation, twisting of Yetter-Drinfeld modules is explored by Andruskiewitsch, Fantino, García and Vendramin in [1]. It should be noted that the twist of a Yetter-Drinfeld module is a functor, in particular a completely canonical construction. 
Section [6 is concluded by an example where working over a ring $R$ which is not a field is essential. We put $R=\mathbb{C} C_{2}=\mathbb{C}[z] /<z^{2}-1>$ and construct a Yetter-Drinfeld module $\left(\widetilde{Y_{n}}\right)_{[1, z]}$ for $R S_{n}$. Here $[1, z]$ denotes a certain non-trivial cocycle in $Z^{2}\left(S_{n}, C_{2}\right)$, which comes from the Schur covering group $T_{n}$ of $S_{n}$. The corresponding Nichols-type algebra $\mathcal{B}\left(\widetilde{Y_{n}}\right)_{[1, z]}$ is a simultaneous cocycle extension of two Nichols algebras over $S_{n}$ that appeared in literature. One is $\mathcal{B}\left(Y_{n}\right)$ (see [5, 6]), related to the Fomin-Kirillov algebra $\mathcal{E}_{n}$, the coinvariant algebra of $S_{n}$ and the rational Cherednik algebra. The other Nichols algebra is $\Lambda_{n}^{w}$, due to Majid [43], related to a quantum differential calculus on the group $S_{n}$. In a recent paper by Vendramin [59] it was shown that these two Nichols algebras are related to each other by a twist by a cocycle (with scalar values).

This prepares the ground for the cocycle extension of the rational Cherednik algebra, constructed at the end of the present paper.

0.10. Algebras with triangular decomposition. In the second part of the paper (sections 7 10) we show how the cocycle extension and cocycle twisting theory can be applied to produce new algebras with triangular decomposition. These are associative algebras with a presentation of a specific form. Suppose that $A$ is an algebra with three chosen subalgebras $A_{-1}, A_{0}$ and $A_{1}$. For $i=-1,0,1$, let $X_{i}$ be a basis of $A_{i}$ over the ground field. If $A_{-1} A_{0}$ and $A_{0} A_{1}$ are subalgebras with bases $X_{-1} X_{0}$ and $X_{0} X_{1}$, respectively, and $X_{-1} X_{0} X_{1}$ is a basis of $A$, then $A$ is said to have triangular decomposition into subalgebras $A_{-1}, A_{0}$ and $A_{1}$.

This property strongly affects the representations $A$, and algebras with triangular decomposition account for many extensively studied objects in modern representation theory. Consider the following examples (over $\mathbb{C}$ ):

— the universal enveloping algebras, $U(\mathfrak{g})$, of semisimple Lie algebras $\mathfrak{g}$;

- their quantisations $U_{q}(\mathfrak{g})$, known as Drinfeld-Jimbo quantum groups;

- and, more recently, double affine Hecke algebras of Cherednik [15] and rational Cherednik algebras of Etingof and Ginzburg [24. Crucially, one can induce representations of $A$ from representations of $A_{0}$. This allows one to work with the category $\mathcal{O}$, defined for each of the above classes of algebras.

The triangular decomposition property puts significant constraints on the relations between the chosen generators $X_{-1} \cup X_{0} \cup X_{1}$ of $A$. For example, the triangular decomposition of $U(\mathfrak{g})$ is a consequence of the Poincaré-Birkhoff-Witt theorem; the latter holds because the defining commutation relations in $U(\mathfrak{g})$ come from a Lie bracket that obeys the Jacobi identity. The role of a (generalised) Jacobi identity is explained by Braverman and Gaitsgory in [13].

To study a broad class of defining relations that lead to a triangular decomposition of $A$, we introduced a class of algebras called braided doubles in [6]. In a braided double $A=A_{-1} A_{0} A_{1}$, the 
middle subalgebra $A_{0}=H$ is a Hopf algebra, and $A_{ \pm 1}$ are graded $H$-module algebras generated in degree 1. The relations between $H$ and $A_{ \pm 1}$ are semidirect product relations. A braided double $A$ is written as

$$
A \cong A_{-1} \otimes H \otimes A_{1}=A_{-1} \rtimes H \ltimes A_{1}
$$

as a vector space, with commutation relations of specific type between $A_{1}$ and $A_{-1}$. We formally define braided doubles below in 7.3 Enveloping algebras and Cherednik algebras in the example above are braided doubles, where the Hopf algebra $H$ is either a universal enveloping algebra of an abelian Lie algebra, $H=U(\mathfrak{h})$, or a group algebra, $H=\mathbb{C} G$. Braided doubles over $\mathbb{C} G$ were further investigated in [7].

0.11. Twists of braided doubles: the key ingredients. In general, a braided double over a group $G$ is a $G$-module algebra but not a $G$-graded algebra, so cannot be twisted by a cocycle on $G$. Our aim is to develop an approach which would produce algebras with triangular decomposition and capture the behaviour of "real" cocycle twists of $G$-graded algebras as fully as possible.

There is a special class of braided doubles, found in [6] and termed the braided Heisenberg doubles. These have triangular decomposition of the form

$$
\mathcal{H}_{Y}=\mathcal{B}(Y) \rtimes \mathbb{C} G \ltimes \mathcal{B}\left(Y^{*}\right),
$$

where $\mathcal{B}(Y), \mathcal{B}\left(Y^{*}\right)$ are Nichols algebras of two dually paired Yetter-Drinfeld modules for $\mathbb{C} G$. To introduce $G$-grading on the braided Heisenberg double, we prove Theorem $8.8, \mathcal{H}_{Y}$ is isomorphic to a semidirect product of $G$ and Majid's braided Weyl algebra of $Y$ [39]. This leads to a non-trivial extension of $G$-grading from $\mathbb{C} G$ onto $\mathcal{H}_{Y}$, as shown in Proposition 8.10,

This allows us to twist a braided Heisenberg double over $G$ by a cocycle of $G$. We stress that this procedure is again functorial and a cocycle twist $\left(\mathcal{H}_{Y}\right)_{\mu}$ of a braided Heisenberg double $\mathcal{H}_{Y}$ is defined in a canonical way.

In contrast to the above, a twist of braided doubles which are not Heisenberg is not functorial. In fact, we do not define a way to twist an abstract braided double $A$. Instead, we consider $A$ together with a braided double morphism

$$
A \stackrel{f}{\rightarrow} \mathcal{H}_{Y}
$$

into a braided Heisenberg double. That such morphisms often exist, and are under favourable conditions injective, is the meaning of the Embedding Theorem 9.4 based on a result from [6].

Assume that the cocycle $\mu$ takes values in some abelian group $\Gamma$. Then every $G$-graded $\mathbb{C}$-algebra $B$ has a cocycle extension $\widetilde{B}_{\mu}$, which is simply the twist by $\mu$ of the $\mathbb{C} \Gamma$-algebra $\mathbb{C} \Gamma \otimes_{\mathbb{C}} B$. Now a $\mu$-extension of the braided double $A$, covering the morphism $f$, is a braided double morphism

$$
\widetilde{A} \stackrel{\widetilde{f}}{\rightarrow}\left(\widetilde{\mathcal{H}_{Y}}\right)_{\mu}
$$


which specialises to $f$ modulo the augmentation ideal $\mathbb{C} \Gamma_{+}$of $\mathbb{C} \Gamma$. Here, $\widetilde{A}$ is required to be just $\mathbb{C} \Gamma \otimes_{\mathbb{C}} A$ as a $\mathbb{C} \Gamma$-module, to reflect what is happening with $G$-graded algebras.

Although a lot of information about the algebra $\widetilde{A}$ can be read off the relations in $\left(\widetilde{\mathcal{H}_{Y}}\right)_{\mu}$, for example the main commutator relation, there are still choices to be made in order to construct $\widetilde{A}$. In fact, the relations in $\widetilde{A}$ are dictated by a particular lift of the $f$-images of generators of $A$ to $\left(\widetilde{\mathcal{H}_{Y}}\right)_{\mu}$. If such a lift is chosen incorrectly, the algebra $\widetilde{A}$ "blows up" and is much bigger than the required $\mathbb{C} \Gamma \otimes_{\mathbb{C}} A$. At the moment, we do not know whether a "correct" lifting exists for every non-trivial cocycle $\mu$.

A cocycle twist of a braided double $A$ by $\mu \in Z^{2}\left(G, \mathbb{C}^{\times}\right)$is defined as a result of taking a cocycle extension of $A$ by a cocycle with values in a finite cyclic group $C_{k}=\left\langle z \mid z^{k}=1\right\rangle$, then specialising to $z=q$ where $q \in \mathbb{C}^{\times}, q^{k}=1$. Here we are again motivated by the properties of cocycle twists of $G$-graded algebras: all of them are quotients of cocycle extensions by cyclic groups, by a result we prove here as Corollary 3.9 .

0.12. Application to rational Cherednik algebras. The last section of the paper, Section 10, is devoted to constructing a cocycle extension of the rational Cherednik algebra $H_{0, c}$ of the group $S_{n}$. By results of [6], there is a morphism

$$
H_{0, c} \stackrel{f}{\rightarrow} \mathcal{H}_{Y_{n}}
$$

where $Y_{n}$ is the Yetter-Drinfeld module for $S_{n}$ mentioned above. We construct an extension of $H_{0, c}$ by the cocycle $\mu=[1, z]$ which covers the morphism $f$. We know the algebra $\left(\widetilde{\mathcal{H}_{Y_{n}}}\right)_{[1, z]}$ from what is done in Section 6, but we need to find a specific lift of the Dunkl elements $\theta_{1}, \ldots, \theta_{n} \in Y_{n}$ to $\left(\widetilde{Y_{n}}\right)_{[1, z]}$. This is achieved by choosing $\widetilde{\theta}_{1}, \ldots, \widetilde{\theta}_{n} \in\left(\widetilde{Y_{n}}\right)_{[1, z]}$ in such a way that their specialisation at $z=-1$ are Majid's flat connections in the Nichols algebra $\Lambda_{n}^{w}$.

The resulting algebra $\widetilde{H}_{0, c}$ over $\mathbb{C} C_{2}$ can be seen to coincide with an algebra constructed by Khongsap and Wang in [36. Our approach via cocycles should work for other imprimitive complex reflection groups $G(m, p, n)$ resulting in covering Cherednik algebras attached to those groups. We leave this generalisation to the upcoming paper 8 .

0.13. Notation. All algebras are associative with 1 . Angular brackets $<>$ denote a two-sided ideal of a given algebra, generated by the elements listed between the brackets. They are not the same as the brackets in the $\langle$ generators $|$ relations $\rangle$ form of a presentation of a group; the context always helps to distinguish between these. We use the symbols $\triangleright$ and $\triangleleft$ to denote a left, respectively right, action of a group $G$ on $X$ where $X$ is a set, an abelian group, a vector space or an algebra; we always assume that $G$ acts by automorphisms of $X$. 


\section{Cocycles, central extensions, the Schur multiplier, twisted group Algebras}

In this section we define 2-cocycles on a finite group $G$ and review the related constructions. Let $\Gamma$ be an abelian group, written multiplicatively. We denote by $\operatorname{Fun}\left(G^{n}, \Gamma\right)$ the set of all functions from $G^{n}$ (the set of all $n$-tuples of elements of $G$ ) to $\Gamma$. We write $C_{k}$ to denote the cyclic group of order $k$. That is, $C_{k}=\left\langle z \mid z^{k}=1\right\rangle$. We write $\mathbb{C}^{\times}$for the multiplicative group $\mathbb{C} \backslash\{0\}$.

1.1. The group $Z^{2}(G, \Gamma)$ of cocycles. The set $\Gamma \times G=\{(z, g): z \in \Gamma, g \in G\}$ has the obvious group structure, the direct product of groups $\Gamma$ and $G$. Suppose that we want to "deform" the group multiplication on $\Gamma \times G$ in such a way that $\Gamma$ is still central. Let $\mu \in F u n(G \times G, \Gamma)$. The product $\star=\star_{\mu}$ on the set $\Gamma \times G$ given by

$$
(z, g) \star\left(z^{\prime}, h\right)=\left(z z^{\prime} \mu(g, h), g h\right) \quad \text { for } z, z^{\prime} \in \Gamma, g, h \in G
$$

is a group multiplication if $\mu$ satisfies the following equations:

$$
\mu(g, h) \mu(g h, k)=\mu(g, h k) \mu(h, k), \quad \mu(1, g)=\mu(g, 1)=1 \quad \text { for all } g, h, k \in G \text {. }
$$

A solution $\mu \in \operatorname{Fun}\left(G^{2}, \Gamma\right)$ of these equations is called a normalised 2-cocycle on $G$ with values in $\Gamma$, see [14, ch. IV, 3]. The abelian group of normalised 2-cocycles with respect to pointwise multiplication is denoted $Z^{2}(G, \Gamma)$.

If $\phi \in \operatorname{Fun}(G, \Gamma)$ is any function such that $\phi(1)=1$, then $d \phi(g, h)=\phi(h) \phi(g h)^{-1} \phi(g)$ is a normalised 2-cocycle called the coboundary of $\phi$. Coboundaries form a subgroup $B^{2}(G, \Gamma) \subset Z^{2}(G, \Gamma)$. Two cocycles $\mu, \nu \in Z^{2}(G, \Gamma)$ that coincide modulo $B^{2}(G, \Gamma)$ are said to be cohomologous. The abelian group

$$
H^{2}(G, \Gamma)=Z^{2}(G, \Gamma) / B^{2}(G, \Gamma)
$$

is the second cohomology group of $G$ with coefficients in $\Gamma$. Readers familiar with cohomology of groups should note that at this stage, the group $\Gamma$ of coefficients has a trivial action of $G$.

1.2. Central extensions of $G$ by $\Gamma$. A central extension of $G$ by $\Gamma$ is a short exact sequence

$$
1 \rightarrow \Gamma \stackrel{\iota}{\rightarrow} E \stackrel{\pi}{\rightarrow} G \rightarrow 1
$$

of groups such that $\iota(\Gamma)$ is a subgroup of the centre of $E$. Central extensions play an important role in group theory, for example in the classification of finite simple groups [17]. Another central extension, $1 \rightarrow \Gamma \stackrel{\iota^{\prime}}{\rightarrow} E^{\prime} \stackrel{\pi^{\prime}}{\rightarrow} G \rightarrow 1$, is equivalent to the one given above if there is a group homomorphism (necessarily an isomorphism) $E \rightarrow E^{\prime}$ which intertwines $\iota, \iota^{\prime}$ and $\pi, \pi^{\prime}$.

A cocycle $\mu \in Z^{2}(G, \Gamma)$ gives rise to a specific central extension of $G$ by $\Gamma$,

$$
1 \rightarrow \Gamma \stackrel{\iota}{\rightarrow} \widetilde{G}_{\mu} \stackrel{\pi}{\rightarrow} G \rightarrow 1 \quad \text { with the group } \widetilde{G}_{\mu}=\left(\Gamma \times G, \star_{\mu}\right)
$$


where $\iota(z)=(z, 1)$ and $\pi((z, g))=g$. We will refer to this central extension, and often to the group $\widetilde{G}_{\mu}$ itself, as the extension of $G$ by the cocycle $\mu$. To simplify notation, we will omit $\iota()$, identifying $z \in \Gamma$ with $(z, 1) \in \widetilde{G}_{\mu}$ and viewing $\Gamma$ as the subgroup $\operatorname{ker} \pi$ of $\widetilde{G}_{\mu}$.

Cohomologous cocycles give rise to equivalent cocycle extensions. Indeed, let $\mu$ and $\nu=\mu d \phi$ be cohomologous cocycles in $Z^{2}(G, \Gamma)$, where $\phi \in F u n(G, \Gamma), \phi(1)=1$. Then the the bijective map $\Gamma \times G \rightarrow \Gamma \times G$, given by $(z, g) \mapsto(z \phi(g), g)$ is a group homomorphism between $\widetilde{G}_{\mu}$ and $\widetilde{G}_{\nu}$, which affords an equivalence of the cocycle extensions $\widetilde{G}_{\mu}$ and $\widetilde{G}_{\nu}$ of $G$.

Conversely, if the two cocycle extensions $\widetilde{G}_{\mu}$ and $\widetilde{G}_{\nu}$ are equivalent, $\mu$ and $\nu$ are cohomologous. By definition, an equivalence of extensions is necessarily a map $\Gamma \times G \rightarrow \Gamma \times G$ which sends $(z, 1)$ to $(z, 1)$ and $(1, g)$ to $(\phi(g), g)$ for some $\phi \in F u n(G, \Gamma), \phi(1)=1$. That this map is a group homomorphism is easily seen to imply that $\nu=\mu d \phi$.

Furthermore, every central extension of $G$ by $\Gamma$ is equivalent to a cocycle extension. Indeed, let $T: G \rightarrow E$ be a set-theoretic section of the extension $1 \rightarrow \Gamma \stackrel{\iota}{\rightarrow} E \stackrel{\pi}{\rightarrow} G \rightarrow 1$. That is, $T$ is a map of sets such that $\pi T=\mathrm{id}_{G}$. We further require $T$ to be a normalised section, that is, $T(1)=1$. Define $\mu \in \operatorname{Fun}\left(G^{2}, \Gamma\right)$ by

$$
T(g) T(h)=\mu(g, h) T(g h) \quad \text { for } g, h \in G,
$$

observing that $T(g) T(h) T(g h)^{-1} \in \operatorname{ker} \pi=\Gamma$. That is, $\mu$ measures the failure of the section $T$ to be a group homomorphism. Then it is easy to see that $\mu \in Z^{2}(G, \Gamma)$ is a normalised 2-cocycle, and the map $\widetilde{G}_{\mu} \rightarrow E$ given by $(z, g) \mapsto z T(g)$, affords an equivalence of the extensions $\widetilde{G}_{\mu}$ and $E$.

The above argument establishes an important fact: the cohomology group $H^{2}(G, \Gamma)$ is in bijection with the equivalence classes of central extensions of $G$ by $\Gamma$. To a normalised cocycle $\mu$ representing a cohomology class in $H^{2}(G, \Gamma)$, the bijection associates the central extension $\widetilde{G}_{\mu}$. In particular, the trivial cocycle $1 \in Z^{2}(G, \Gamma)$ corresponds to the split extension $\widetilde{G}=\Gamma \times G$. This bijection is a particular case of Schreier's theorem, see [49, Theorem 7.34].

1.3. The Schur multiplier of $G$. Cocycle extensions of $G$ yield groups larger than $G$. A variation of the above construction can be used to deform the multiplication in the group algebra $\mathbb{C} G$ of $G$. This is explained below in 1.5 the input is a normalised 2-cocycle on $G$ with values in $\mathbb{C}^{\times}$. Such cocycles, modulo coboundaries, form an important finite abelian group

$$
M(G):=H^{2}\left(G, \mathbb{C}^{\times}\right)
$$

known as the Schur multiplier of the finite group $G$. (In the case where $G$ is infinite, which we do not consider here, there are competing non-equivalent definitions of the Schur multiplier of $G$.)

Example 1.4 (Schur multiplier of a finite abelian group). To compute $M(G)$ where $G$ is a finite abelian group, one can use an alternative definition of $M(G)$ via group homology, $M(G)=H_{2}(G, \mathbb{Z})$, 
see [9, ch. I, Proposition 5.5]. By [14, Theorem 6.4(iii)], this is the same as the abelian group $\wedge^{2} G$, the quotient of the group $G \otimes_{\mathbb{Z}} G$ by the subgroup generated by $g \otimes_{\mathbb{Z}} g, g \in G$. The group $G$ is non-canonically isomorphic to $G^{*}=\left\{\right.$ homomorphisms from $G$ to $\left.\mathbb{C}^{\times}\right\}$, and a more careful analysis identifies $M(G)$ with $\wedge^{2} G^{*}$ which is the group of all bicharacters $\mu \in \operatorname{Fun}\left(G^{2}, \mathbb{C}^{\times}\right)$modulo the group of symmetric bicharacters. A function $\mu: G^{2} \rightarrow \mathbb{C}^{\times}$is a bicharacter, if $\mu(g,-)$ and $\mu(-, g)$ are homomorphisms $G \rightarrow \mathbb{C}^{\times}$for all $g \in G$; it is symmetric if $\mu(g,-)=\mu(-, g)$ for all $g$. Bicharacters form an abelian group with respect to pointwise multiplication.

To give an explicit example, consider $G=C_{p}^{n}$ where $p$ is prime. A convenient presentation of $G$ is

$$
G=\left\langle\gamma_{1}, \ldots, \gamma_{n} \mid \gamma_{i}^{p}=1, \gamma_{i} \gamma_{j}=\gamma_{j} \gamma_{i}, 1 \leq i, j \leq n\right\rangle .
$$

Let $\omega$ be a primitive $p$ th root of unity in $\mathbb{C}^{\times}$. If $1 \leq i<j \leq n$, denote by $b_{i j}$ the bicharacter of $G$ defined on generators by

$$
b_{i j}\left(\gamma_{k}, \gamma_{l}\right)= \begin{cases}\omega, & \text { if }(k, l)=(j, i), \\ 1, & \text { if }(k, l) \neq(j, i) .\end{cases}
$$

It is easy to see that the bicharacters $b_{i j}, 1 \leq i<j \leq n$, generate an abelian group isomoprhic to

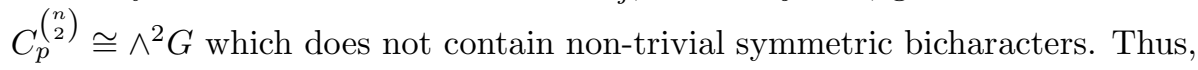

$$
M\left(C_{p}^{n}\right) \cong C_{p}^{\left(\begin{array}{c}
n \\
2
\end{array}\right)}
$$

Schur multipliers of many finite non-abelian groups are known; see in particular [32] for Schur multipliers of all finite simple groups. The Schur multipliers of symmetric groups and alternating groups were determined by Schur in [54.

1.5. Twisted group algebras. Using a cocycle $\nu \in Z^{2}\left(G, \mathbb{C}^{\times}\right)$, one can construct a twisted group algebra (a cocycle twist $\mathbb{C} G_{\nu}$ of the group algebra $\mathbb{C} G$ by $\nu$ ). The underlying vector space of $\mathbb{C} G_{\nu}$ is $\mathbb{C} G$, and the associative multiplication $\star_{\nu}$ is defined on the basis $\{g \mid g \in G\}$ by $g \star_{\nu} h=$ $\nu(g, h) g h$. This is the same formula as in the cocycle extension, but the values of $\nu$ are viewed as scalars. Cohomologous cocycles lead to isomorphic twists of $\mathbb{C} G$, thus twisted group algebras are parametrised, up to isomorphism, by elements of the Schur multiplier $M(G)$ of $G$.

In a sense, cocycle extensions of $G$ are more general than cocycle twists of $\mathbb{C} G$, because any cocycle twist of $\mathbb{C} G$ is a quotient of the group algebra of an extension of $G$ by a cyclic group:

Lemma 1.6. Let $G$ be a finite group and $\nu \in Z^{2}\left(G, \mathbb{C}^{\times}\right)$. There exist a finite cyclic group $C_{m}=$ $\left\langle z \mid z^{m}=1\right\rangle$, a cocycle $\mu \in Z^{2}\left(G, C_{m}\right)$ and an mth root of unity $q \in \mathbb{C}^{\times}$, such that

$$
\mathbb{C} G_{\nu} \cong \mathbb{C} \widetilde{G}_{\mu} /<z-q>\text {. }
$$


Proof. By [49, lemma 7.65], the subgroup $B^{2}\left(G, \mathbb{C}^{\times}\right)$of $Z^{2}\left(G, \mathbb{C}^{\times}\right)$has a complement $M$ which is a finite subgroup of $Z^{2}\left(G, \mathbb{C}^{\times}\right)$isomorphic to $H^{2}\left(G, \mathbb{C}^{\times}\right)$. That is, $Z^{2}\left(G, \mathbb{C}^{\times}\right)$is a direct product of $M$ and $B^{2}\left(G, \mathbb{C}^{\times}\right)$. Let $\sigma \in M$ be the cocycle cohomologous to $\nu$ so that $\mathbb{C} G_{\nu} \cong \mathbb{C} G_{\sigma}$, and let $m$ be the order of $\sigma$ in the finite group $M$. Then all values of $\sigma$ are $m$ th roots of unity in $\mathbb{C}^{\times}$, hence of the form $q^{k}$ where $q$ is a primitive $m$ th root of 1 and $0 \leq k \leq m-1$. Define $\mu \in Z^{2}\left(G, C_{m}\right)$ by $\mu(g, h)=z^{k}$ whenever $\sigma(g, h)=q^{k}$. This guarantees that the map $\pi_{q}: \mathbb{C} \widetilde{G}_{\mu} \rightarrow \mathbb{C} G_{\sigma}$ given by $\left(z^{k}, g\right) \mapsto q^{k} g$ is an algebra homomorphism. It is surjective and its kernel contains $z-q \cdot 1$, so $\operatorname{dim} \mathbb{C} \widetilde{G}_{\mu} /<z-q>\geq \operatorname{dim} \mathbb{C} \widetilde{G}_{\mu} / \operatorname{ker} \pi_{q}=|G|$. On the other hand, $\left(z^{k}, g\right)$ is the same as $q^{k}(1, g)$ in $\mathbb{C} \widetilde{G}_{\mu} /<z-q>$, hence $\operatorname{dim} \mathbb{C} \widetilde{G}_{\mu} /<z-q>\leq|G|$. We conclude that $\operatorname{ker} \pi_{q}=\langle z-q>$ so that $\mathbb{C} \widetilde{G}_{\mu} /<z-q>\cong \mathbb{C} G_{\sigma} \cong \mathbb{C} G_{\nu}$.

1.7. Example: the Clifford algebra is a twisted group algebra. Let $A$ be the group algebra of the group $K=C_{2}^{n}$. That is, $A$ is generated by $\gamma_{1}, \ldots, \gamma_{n}$ subject to relations $\gamma_{i}^{2}=1$ and $\gamma_{i} \gamma_{j}=\gamma_{j} \gamma_{i}$ for all $i, j \in\{1, \ldots, n\}$. There is another, noncommutative, associative product $\star$ on the vector space $A$ such that

$$
\gamma_{i} \star \gamma_{i}=1, \quad \gamma_{i} \star \gamma_{j}=\gamma_{i} \gamma_{j}, \quad \gamma_{j} \star \gamma_{i}=-\gamma_{i} \gamma_{j}, \quad 1 \leq i<j \leq n .
$$

Note that $(A, \star)$ is the Clifford algebra of rank $n$ (named after W. K. Clifford). It is a cocycle twist of $\mathbb{C} K$ by the cocycle $\mu \in Z^{2}\left(K, \mathbb{C}^{\times}\right)$given on generators by

$$
\mu\left(\gamma_{i}, \gamma_{j}\right)= \begin{cases}+1 & \text { if } i \leq j \\ -1 & \text { if } i>j\end{cases}
$$

and extended to $K \times K$ as a bicharacter. In the notation of Example 1.4, the cocycle $\mu$ is $\prod_{1 \leq i<j \leq n} b_{i j}$.

As prescribed by Lemma 1.6, the Clifford algebra is the quotient, modulo the relation $z=-1$, of the group algebra of a non-abelian central extension of $C_{2}^{n}$ by $C_{2}=\{1, z\}$.

\section{The Schur MUltiplier of $S_{n}$}

We now describe the above constructions more explicitly in the case of $G=S_{n}$, the symmetric group of degree $n$. Historically, this was the first group for which the Schur multiplier was found; see Schur [52, 53, 54].

2.1. Projective representations. Initially, let $G$ be an arbitrary finite group. Projective representations of $G$ are closely associated to central extensions of $G$ and 2-cocycles on $G$. Let $V$ be a finite-dimensional vector space over $\mathbb{C}$. A projective representation of $G$ on $V$ is a homomorphism $\rho: G \rightarrow \operatorname{PGL}(V)$ where $\operatorname{PGL}(V)$ is the quotient group of $\mathrm{GL}(V)$ modulo its centre $\mathbb{C}^{\times} \cdot \mathrm{id}_{V}$. By 
choosing representatives for $\rho(g), g \in G$, in $\mathrm{GL}(V)$ such that $\rho(1)=\mathrm{id}_{V}$ - or choosing a normalised section $\operatorname{PGL}(V) \rightarrow \mathrm{GL}(V)$ - we may as well regard $\rho$ as a map $G \rightarrow \mathrm{GL}(V)$ which satisfies

$$
\rho(g) \rho(h)=\mu(g, h) \rho(g h), \quad g, h \in G,
$$

for a function $\mu \in \operatorname{Fun}\left(G^{2}, \mathbb{C}^{\times}\right)$called a normalised factor set. The associative law implies that $\mu \in Z^{2}\left(G, \mathbb{C}^{\times}\right)$. Thus, normalised factor set is just another name for a normalised 2-cocycle with values in $\mathbb{C}^{\times}$.

It is easy to see that projective representations of $G$ with a given factor set $\mu$ are the same as representations of the twisted group algebra $\mathbb{C} G_{\mu}$, introduced above.

Projective representations arise naturally in the representation theory of groups. An example is the Clifford theory which deals with extending a representation of a normal subgroup $H$ of $G$ to $G$, see the paper [16] by A. H. Clifford.

2.2. Schur covers. A way to construct projective representations of $G$ is as follows. Let $1 \rightarrow \Gamma \rightarrow$ $E \rightarrow G \rightarrow 1$ be a central extension of $G$ with normalised section $T: G \rightarrow E$. Let $r: E \rightarrow \mathrm{GL}(V)$ be a representation of $E$. Then $\rho=r T: G \rightarrow \mathrm{GL}(V)$ is a projective representation of $G$. In this case, it is said that the projective representation $\rho$ of $G$ is lifted to the (linear) representation $r$ of $E$.

By a result of Schur [52], for any finite group $G$ there exists at least one central extension

$$
1 \rightarrow \Gamma \hookrightarrow E \rightarrow G \rightarrow 1
$$

of $G$, such that all the projective representations of $G$ are lifted to $E$. Extensions with this projective lifting property where the order of $E$ is smallest possible are known as Schur covers of $G$, while the group $E$ is termed a Schur covering group, or a representation group of $G$.

Equivalently, a central extension is a Schur cover if it is a stem extension and $\Gamma \cong M(G)$. Stem extension means that $\Gamma \subseteq[E, E]$, the derived subgroup of $E$. This and other equivalent characterisations of Schur covers can be found in [9, ch. II, III] and [49, ch. 7].

2.3. Group algebras of Schur covering groups. Two Schur covers of $G$ may not be equivalent as extensions, and moreover not isomorphic as abstract groups. The simplest example is

$$
G=C_{2} \times C_{2},
$$

the Klein 4-group. By Example 1.4,

$$
M\left(C_{2} \times C_{2}\right)=C_{2} .
$$

The group $G$ has two non-isomorphic Schur covering groups: $D_{8}$, the dihedral group of order 8 , and $Q_{8}$, the quaternion group, see [49, Example 7.17]. 
Nevertheless, any two Schur covers of $G$ are isoclinic to each other. Isoclinism of abstract groups, introduced by P. Hall [34, is an equivalence relation weaker that isomorphism: for example, all abelian groups form one isoclinism class. See [9, ch. III] for the definition of isoclinism of abstract groups and of central extensions.

We will need the following observation about group algebras of isoclinic groups. Let $H_{1}, H_{2}$ be two finite groups, and let $m_{i}(n)$ denote the number of irreducible complex characters of the group $H_{i}$ of degree $n$. If $H_{1}$ is isoclinic to $H_{2}$, then by [9, Corollary 5.8], $m_{1}(n)\left|H_{2}\right|_{p}=m_{2}(n)\left|H_{1}\right|_{p}$ for all $n$ and for all primes $p$. In particular, if $H_{1}, H_{2}$ have the same order, $m_{1}(n)=m_{2}(n)$ for all $n$.

Recall from the representation theory of finite groups over $\mathbb{C}$ that the group algebra $\mathbb{C} H_{i}$ is isomorphic to the direct sum of matrix algebras of size $d \times d$ where $d$ runs over the multiset of degrees of irreducible characters of $H_{i}$. That is, the function $m_{i}(n)$ determines the isomorphism class of the group algebra $\mathbb{C} H_{i}$. We conclude that if $H_{1}$ and $H_{2}$ are isoclinic groups of the same finite order, $\mathbb{C} H_{1} \cong \mathbb{C} H_{2}$.

In particular, this isomorphism holds if $H_{1}$ and $H_{2}$ are Schur covering groups of the same finite group $G$. Another way to explain the equality of $m_{1}(n)$ and $m_{2}(n)$ in this case is to observe that irreducible representations of $H_{i}$ are, by the projective lifting property, precisely the irreducible projective representations of $G$. Hence we obtain the following

Lemma 2.4. Let $G$ be a finite group. The group algebras, over $\mathbb{C}$, of all Schur covering groups of $G$ are pairwise isomorphic.

2.5. The Schur covering groups of $S_{n}$. Let now $G=S_{n}$. We describe two non-equivalent covers of $S_{n}, n \geq 4$, found by Schur [54] in 1911. The importance of a Schur cover for us is that it allows us to choose a normalised section and to compute a non-trivial 2-cocycle on $S_{n}$ with values in $M\left(S_{n}\right)$. Schur found that $M\left(S_{n}\right)$ is trivial if $n \leq 3$ and

$$
M\left(S_{n}\right)=C_{2} \quad \text { if } n \geq 4 .
$$

It can be shown that there are no non-trivial cocycles on $S_{n}$ with values in $C_{p}, p$ prime, $p \neq 2$.

As was explained above in 1.2, central extensions of $S_{n}$ by

$$
C_{2}=\{1, z\}
$$

are parametrised, up to equivalence, by the cohomology group $H^{2}\left(S_{n}, C_{2}\right)$. One can show, in the same fashion as Schur did in [54, that a cocycle $\mu: S_{n} \times S_{n} \rightarrow C_{2}$ can be chosen, up to a coboundary, so that for all $i, k, l \in\{1, \ldots, n-1\}, k<l$,

$$
\mu\left(s_{i}, s_{i}\right)=\alpha, \quad \mu\left(s_{k}, s_{l}\right)=1, \quad \mu\left(s_{l}, s_{k}\right)=\beta, \quad \alpha, \beta \in\{1, z\}
$$


Here $s_{i}=(i i+1)$ is a simple transposition. It follows that

$$
H^{2}\left(S_{n}, C_{2}\right) \cong C_{2} \times C_{2} \quad \text { for } n \geq 4,
$$

where a cohomology class in $H^{2}\left(S_{n}, C_{2}\right)$ is represented by $[\alpha, \beta]$. We will abuse the notation slightly and write the cohomology class $[\alpha, \beta]$ instead of its representative $\mu$. The four pairwise non-equivalent extensions of $S_{n}$ by $C_{2}$ are then as described in

Theorem 2.6 (Schur [54]). If $\alpha, \beta \in\{1, z\}$, the group

$$
\begin{aligned}
\left(\widetilde{S_{n}}\right)_{[\alpha, \beta]}=\left\langle t_{1}, \ldots, t_{n-1}, z\right| & t_{i}^{2}=\alpha, z^{2}=1, z t_{i}=t_{i} z, \\
& t_{j} t_{j+1} t_{j}=t_{j+1} t_{j} t_{j+1}, t_{k} t_{l}=\beta t_{l} t_{k}: \\
& 1 \leq i \leq n-1,1 \leq j \leq n-2,1 \leq k<l-1 \leq n-2\rangle
\end{aligned}
$$

is a central extension of $S_{n}$ by $C_{2}$ via the map $\left(\widetilde{S_{n}}\right)_{[\alpha, \beta]} \rightarrow S_{n}$ given by $t_{i} \mapsto(i i+1), z \mapsto 1$. The groups $\left(\widetilde{S_{n}}\right)_{[1, z]}$ and $\left(\widetilde{S_{n}}\right)_{[z, z]}$ are Schur covers of $S_{n}$, whereas $\left(\widetilde{S_{n}}\right)_{[1,1]}$ and $\left(\widetilde{S_{n}}\right)_{[z, 1]}$ are not stem extensions of $S_{n}$.

To clarify the notation used, recall from 1.2 that as a set, the group $\left(\widetilde{S_{n}}\right)_{[\alpha, \beta]}$ is the cartesian product $C_{2} \times S_{n}$. It is generated by $z=(z, 1)$ and by $t_{i}=\left(1, s_{i}\right)$.

Let us briefly comment on why the cocycles denoted $[1,1]$ and $[z, 1]$ do not give rise to stem extensions of $S_{n}$. Clearly, $\left(\widetilde{S_{n}}\right)_{[1,1]}=S_{n} \times C_{2}$ is the trivial extension. One can show that the cocycle $[z, 1]$ is given by the formula

$$
[z, 1](g, h)=z^{(\ell(g)+\ell(h)-\ell(g h)) / 2}, \quad g, h \in S_{n} .
$$

Here $\ell(g)$ is the length (the number of inversions) of the permutation $g$, and one notes that $\ell(g)+$ $\ell(h)-\ell(g h)$ is always even. Write $S_{n}$ as $A_{n} \rtimes C_{2}$ where $A_{n}$ is the alternating group and $C_{2}=$ $\{\mathrm{id},(12)\}$. Then $\left(\widetilde{S_{n}}\right)_{[z, 1]}=A_{n} \rtimes C_{4}$ is obtained in a straightforward way from the extension $1 \rightarrow C_{2} \rightarrow C_{4} \rightarrow C_{2} \rightarrow 1$. Both for $\left(\widetilde{S_{n}}\right)_{[1,1]}$ and for $\left(\widetilde{S_{n}}\right)_{[z, 1]}$, the element $z$ lies in the kernel of the extension but not in the derived subgroup.

One is left with two non-isomorphic Schur covering groups of $S_{n}$ (assuming $n \geq 4$ ): in Schur's notation,

$$
T_{n}=\left(\widetilde{S_{n}}\right)_{[1, z]}, \quad T_{n}^{\prime}=\left(\widetilde{S_{n}}\right)_{[z, z]} .
$$

By Lemma 2.4.

$$
\mathbb{C} T_{n} \cong \mathbb{C} T_{n}^{\prime} \quad \text { (this isomorphism is not canonical). }
$$


2.7. The spin symmetric group. Let us now apply the cocycle twist to deform the associative product on the group algebra $\mathbb{C} S_{n}$. Such a twist is given, up to an algebra isomorphism, by an element of $H^{2}\left(S_{n}, \mathbb{C}^{\times}\right)=C_{2}$. Note that the map

$$
H^{2}\left(S_{n}, C_{2}\right) \rightarrow H^{2}\left(S_{n}, \mathbb{C}^{\times}\right), \quad z \mapsto-1
$$

is not injective. Indeed, although the cocycle $[z, 1] \in Z^{2}\left(S_{n}, C_{2}\right)$ considered above is not cohomologous to the trivial cocycle $[1,1]$, its image in $Z^{2}\left(S_{n}, \mathbb{C}^{\times}\right)$can be written as $\mathrm{i}^{\ell(g)} \mathrm{i}^{-\ell(g h)} \mathrm{i}^{\ell(h)}$ where $\mathrm{i}=\sqrt{-1} \in \mathbb{C}^{\times}$. This is clearly a coboundary. Therefore, up to isomorphism, there is only one non-trivial cocycle twist of $\mathbb{C} S_{n}$ :

$$
\left(\mathbb{C} S_{n}\right)_{[1,-1]}=\mathbb{C} T_{n} /<z+1>\cong\left(\mathbb{C} S_{n}\right)_{[-1,-1]}=\mathbb{C} T_{n}^{\prime} /<z+1>.
$$

Although the isomorphism between $\mathbb{C} T_{n}$ and $\mathbb{C} T_{n}^{\prime}$ is not explicit, there is a straightforward isomorphism between the quotients $\mathbb{C} T_{n} /<z+1>$ and $\mathbb{C} T_{n}^{\prime} /<z+1>$ which sends the generator $t_{j}$ of $\mathbb{C} T_{n} /<z+1>$ to it $t_{j}^{\prime}$ in $\mathbb{C} T_{n}^{\prime} /<z+1>$.

The twisted group algebra $\left(\mathbb{C} S_{n}\right)_{[1,-1]} \cong\left(\mathbb{C} S_{n}\right)_{[-1,-1]}$ is referred to as the spin symmetric group in Wang [60].

2.8. The 1-cocycle $\chi_{\mu}$ and its calculation for $S_{n}$. Given a cocycle $\mu \in Z^{2}(G, \Gamma)$ on a finite group $G$, we are interested in the values of the function

$$
\chi_{\mu}(g, h)=\mu(g, h) \mu\left(g h g^{-1}, g\right): G \times G \rightarrow \Gamma .
$$

This formula for $\chi_{\mu}(g, h)$ guarantees that the function

$$
G \rightarrow \operatorname{Fun}(G, \Gamma), \quad g \mapsto \chi_{\mu}(g,-)
$$

is a 1-cocycle, where $\operatorname{Fun}(G, \Gamma)$ is viewed as a right $G$-module via the adjoint action of $G$ on itself. (We have not defined cocycles with coefficients in a non-trivial module, but see the definition in [14, ch. III, 1, example 3].) We thus have a group homomorphism

$$
Z^{2}(G, \Gamma) \rightarrow Z^{1}(G, \operatorname{Fun}(G, \Gamma)), \quad \mu \mapsto \chi_{\mu} .
$$

It is not difficult to check that it induces a homomorphism

$$
H^{2}(G, \Gamma) \rightarrow H^{1}(G, \operatorname{Fun}(G, \Gamma)) .
$$

The reason why we are interested in this homomorphism will become apparent later in Section 6 and has to do with twisting Yetter-Drinfeld modules.

We have just analysed the group $H^{2}\left(S_{n}, C_{2}\right)$, so now is a good point to calculate $\chi_{\mu}$ for future use. For $\mu=[1, z]$ in the notation of 2.5, the corresponding cocycle in $Z^{1}\left(S_{n}, \operatorname{Fun}\left(S_{n}, C_{2}\right)\right)$ was explicitly found by Vendramin [59, Lemma 3.7 and Theorem 3.8]: 
Theorem 2.9 (Vendramin). If $\sigma, \tau$ are transpositions in $S_{n}, \tau=(i j), i<j$, then

$$
\chi_{[1, z]}(\sigma, \tau)= \begin{cases}z, & \text { if } \sigma(i)<\sigma(j), \\ 1, & \text { if } \sigma(i)>\sigma(j) .\end{cases}
$$

The expression for $\chi_{[z, z]}(\sigma, \tau)$ is more complicated: one can check that

$$
\chi_{[z, z]}(\sigma, \tau)=z^{\left(\ell\left(\sigma \tau \sigma^{-1}\right)-\ell(\tau)\right) / 2} \chi_{[1, z]}(\sigma, \tau) .
$$

\section{Cocycle twists And COCyCle EXtensions of GROUP-GRADED ALGEBRAS}

Definition 3.1 ( $G$-graded algebra over $R$ ). Let $R$ be a commutative ring and $A$ be an associative algebra over $R$. We say that $A$ is graded by a finite group $G$, if $A=\bigoplus_{g \in G} A_{g}$, where $\oplus$ means a direct sum of $R$-modules; $A_{g} A_{h} \subset A_{g h}$ for all $g, h \in G$; and the identity element of $A$ is in $A_{1}$.

Example 3.2. The group algebra $R G$ is $G$-graded, with $(R G)_{g}=R g$.

3.3. Cocycle twists of $A$. Let $A$ be a $G$-graded algebra over $R$. The multiplication in $A$ can be twisted by a 2-cocycle on $G$ with values in $R^{\times}$. Every cocycle $\mu \in Z^{2}\left(G, R^{\times}\right)$gives rise to a new associative product $\star=\star_{\mu}$ on the underlying $R$-module $A$ given by

$$
a \star_{\mu} b=\mu(g, h) a b \quad \text { for } a \in A_{g}, b \in A_{h}, g, h \in G .
$$

We refer to the algebra $A_{\mu}=\left(A, \star_{\mu}\right)$ as the cocycle twist of $A$ by $\mu$.

3.4. Realisation of the cocycle twist via coaction. The twists of the group algebra of $G$, discussed in the previous section, are in a sense the main example of cocycle twists of a groupgraded algebra. We will now explain this. We will write the $G$-grading as a coaction:

Definition 3.5 (Coaction). If $V$ is a $G$-graded $R$-module, $V=\bigoplus_{g \in G} V_{g}$, the coaction of $G$ on $V$ is the $R$-module map

$$
\delta: V \rightarrow V \underset{R}{\otimes} R G, \quad \delta(v)=v \underset{R}{\otimes} g \quad \text { if } v \in V_{g} .
$$

It follows from the definition of a $G$-graded algebra that the coaction $\delta: A \rightarrow A \otimes_{R} R G$ is a homomorphism of algebras over $R$, where $A \otimes_{R} R G$ is viewed as a tensor product of two algebras (the tensor factors commute).

Now let $\mu \in Z^{2}\left(G, R^{\times}\right)$. View the codomain of $\delta$ as the algebra $A \otimes_{R} R G_{\mu}$ where $A$ and $R G_{\mu}$ commute.

Lemma 3.6. $\delta$ is an algebra isomorphism between $\left(A, \star_{\mu}\right)$ and the subalgebra $\delta(A)$ of $A \otimes_{R} R G_{\mu}$. 
Proof. Note that $\delta$ is injective because $\left(\operatorname{id}_{A} \otimes_{R} \epsilon\right) \delta=\operatorname{id}_{A}$ where $\epsilon: R G \rightarrow R$ is the augmentation map. It is enough to check that $\delta(a b)=\delta(a) \delta(b)$ where $a \in A_{g}, b \in A_{h}$. Both sides are equal to $a b \otimes_{R} g h$.

In other words, it is enough to twist $R G$, and the twisted product on every $G$-graded $R$-algebra is induced via the coaction.

3.7. Cocycle extensions of $\mathbb{C}$-algebras. In the rest of this section, we will look at the case where $R$ is a commutative algebra over $\mathbb{C}$. Let $A_{0}$ be a $G$-graded algebra over $\mathbb{C}$, and let $\mu \in Z^{2}\left(G, R^{\times}\right)$. The cocycle extension of $A_{0}$ by $\mu$ is an algebra over $R$. It can be constructed in two steps. First, consider $R \otimes_{\mathbb{C}} A_{0}$ as an algebra over $R$, with the $G$-grading coming from $A_{0}$. The algebra $R \otimes_{\mathbb{C}} A_{0}$ is a trivial extension of $A_{0}$ (extension of scalars with no cocycle involved).

Then twist the multiplication in $R \otimes_{\mathbb{C}} A_{0}$ as in 3.3, obtaining $\left(R \otimes_{\mathbb{C}} A_{0}\right)_{\mu}$. We denote the $R$-algebra $\left(R \otimes_{\mathbb{C}} A_{0}\right)_{\mu}$ by $\left(\widetilde{A}_{0}\right)_{\mu}$ and call it the extension of $A_{0}$ by the cocycle $\mu$. Note that a cocycle extension of a $\mathbb{C}$-algebra by a cocycle with values in $R^{\times}$is always free as an $R$-module.

3.8. Flat $\Gamma$-deformations of $\mathbb{C}$-algebras. Specialisation. Let $A$ be an algebra over $\mathbb{C}$ and $\Gamma$ be an abelian group. We further restrict $R$ to being the group algebra $\mathbb{C} \Gamma$. A flat $\Gamma$-deformation of $A$ is an algera $\widetilde{A}$ over the ring $\mathbb{C} \Gamma$ which is a free $\mathbb{C} \Gamma$-module, together with an isomorphism $\widetilde{A} / \mathbb{C} \Gamma_{+} \widetilde{A} \stackrel{\sim}{\rightarrow} A$ of algebras. Here $\mathbb{C} \Gamma_{+}$is the augmentation ideal of $\mathbb{C} \Gamma$, that is, the subspace of $\mathbb{C} \Gamma$ spanned by $\{z-1 \mid z \in \Gamma\}$. Note that $\mathbb{C} \Gamma_{+} \widetilde{A}$ is a two-sided ideal of $\widetilde{A}$. Cf. [23, Definition 1.1]. Any algebra $A$ has a trivial flat $\Gamma$-deformation $\mathbb{C} \Gamma \otimes_{\mathbb{C}} A$.

In the case where $\Gamma$ is a cyclic group $C_{m}=\left\langle z \mid z^{m}=1\right\rangle$, it is convenient to denote the algebra $\widetilde{A} / \mathbb{C} \Gamma_{+} \widetilde{A}$ by $\left.\widetilde{A}\right|_{z=1}$. More generally, for any $q \in \mathbb{C}^{\times}$such that $q^{m}=1$, one has the algebra

$$
\left.\widetilde{A}\right|_{z=q}:=\widetilde{A} /(z-q) \widetilde{A}
$$

over $\mathbb{C}$. The algebra $\left.\widetilde{A}\right|_{z=q}$ is the specialisation of $\widetilde{A}$ at $z=q$.

Note that any extension of $A$ by a cocycle $\mu \in Z^{2}(G, \Gamma)$ is a flat $\Gamma$-deformation of $A$. Lemma 1.6 and Lemma 3.6 now imply

Corollary 3.9. For every cocycle $\nu \in Z^{2}\left(G, \mathbb{C}^{\times}\right)$, there exists a finite cyclic group $C_{m}=\left\langle z \mid z^{m}=1\right\rangle$, a cocycle $\mu \in Z^{2}\left(G, C_{m}\right)$ and an mth root of unity $q \in \mathbb{C}^{\times}$such that the twist $A_{\nu}$ of $A$ is isomorphic to a specialisation of $\widetilde{A}_{\mu}$ at $z=q$.

\section{Cocycle twists And the LAZY COHOMOlogy of A MONOIDAl CATEGORY}

A twist of $G$-graded algebras by a cocycle representing an element of $M(G)$ can be viewed as an endofunctor of the category of $G$-graded algebras over $\mathbb{C}$. The cocycle extension, too, should be understood as a functor, but between two different categories of algebras. This motivates us to 
review the necessary formalism of algebras in monoidal categories. We show how a 2-cocycle can be defined categorically as a natural automorphism of the monoidal product on a category $\mathcal{C}$; it induces an endofunctor on the category $\mathcal{C}-\mathrm{Alg}$ of algebras in $\mathcal{C}$.

4.1. Algebras in a monoidal category. Recall that a category $\mathcal{C}$ is monoidal if it is equipped with a monoidal product bifunctor $\otimes: \mathcal{C} \times \mathcal{C} \rightarrow \mathcal{C}$ and a unit object $\mathbb{I}$ that satisfy the axioms in Mac Lane [38, ch. VII]. The first section of Deligne - Milne article [19] also provides a clear and concise introduction to monoidal categories. A standard example is $R$-Mod where $R$ is a commutative ring, with $\otimes=\otimes_{R}$ and $\mathbb{I}=R$. All monoidal categories $\mathcal{C}$ that we consider are equipped with a faithful functor to $R$-Mod which preserves the monoidal product and the unit object. In particular, the associativity constraint $\Phi: \otimes \circ\left(\otimes \times \operatorname{id}_{\mathcal{C}}\right) \cong \otimes \circ\left(\operatorname{id}_{\mathcal{C}} \times \otimes\right)$ is canonical and will be omitted from formulae. We explicitly consider the most basic case where $R \cong \mathbb{C} \oplus \mathbb{C} \oplus \ldots \oplus \mathbb{C}$ is the group algebra $\mathbb{C} \Gamma$ of a finite abelian group $\Gamma$, and in the main applications we will have $\Gamma=C_{2}$.

An algebra (or monoid) in a monoidal category $\mathcal{C}$ is a triple $(A, m, \eta)$ where $A \in \mathrm{Ob} \mathcal{C}$ and the multiplication morphism $m: A \otimes A \rightarrow A$ and the unit morphism $\eta: \mathbb{I} \rightarrow A$ satisfy the usual associativity and unitality axioms. See [38, ch. VII, 3]. Morphisms between algebras in $\mathcal{C}$ are $\mathcal{C}$-morphisms between the underlying objects that intertwine the multiplication morphisms and the unit morphisms. Thus, algebras in a monoidal category $\mathcal{C}$ form a category $\mathcal{C}$ - Alg equipped with the forgetful faithful functor $(A, m, \eta) \mapsto A$ from $\mathcal{C}$ - Alg to $\mathcal{C}$. For example, $R$ - Mod - Alg is the category of algebras over the commutative ring $R$.

4.2. Example: the free algebra of an object of $\mathcal{C}$. Assume that the monoidal category $\mathcal{C}$ has countable direct sums which are preserved by the monoidal structure: that is, $X \otimes \bigoplus_{i \in \mathbb{N}} Y_{i}$ is a direct sum of $\left\{X \otimes Y_{i} \mid i \in \mathbb{N}\right\}$. Then every object $V \in \mathrm{Ob} \mathcal{C}$ gives rise to a free algebra $T(V) \in \mathrm{Ob} \mathcal{C}-\mathrm{Alg}$. As an object in $\mathcal{C}$,

$$
T(V)=\bigoplus_{n \in \mathbb{N}} V^{\otimes n}
$$

where the tensor powers are defined inductively by $V^{\otimes 0}=\mathbb{I}$ and $V^{\otimes n}=V^{\otimes n-1} \otimes V$. By definition of a direct sum, a morphism $f: T(V) \rightarrow X$ in $\mathcal{C}$ is the same as a collection of morphisms $\left.f\right|_{V \otimes n}: V^{\otimes n} \rightarrow$ $X$, so the multiplication on the tensor algebra is the unique morphism $m: T(V) \otimes T(V) \rightarrow T(V)$ such that $\left.m\right|_{V \otimes m} \otimes V \otimes n$ is the canonical isomorphism onto $V^{\otimes m+n}$ given by the associativity of $\otimes$. See [38, ch. VII, 3, Theorem 2].

Observe that $T$ is a functor from $\mathcal{C}$ to $\mathcal{C}$ - Alg. If $f: V \rightarrow W$ is a morphism in $\mathcal{C}$, then

$$
T(f): T(V) \rightarrow T(W),\left.\quad T(f)\right|_{V \otimes n}=f^{\otimes n},
$$

is the corresponding morphism in $\mathcal{C}$-Alg. 
The following notion of a 2-cocycle on a monoidal category $\mathcal{C}$ is the same as laycle (lazy cocycle) in [47.

Definition 4.3 (cocycle). Let $\mu: \otimes \rightarrow \otimes$ be an automorphism of the monoidal product on $\mathcal{C}$; that is, a family of automorphisms $\mu_{X, Y}: X \otimes Y \rightarrow X \otimes Y$ natural in $X, Y$. It is a normalised 2-cocycle on $\mathcal{C}$ if

$$
\mu_{X, Y} \otimes Z\left(\operatorname{id}_{X} \otimes \mu_{Y, Z}\right)=\mu_{X \otimes Y, Z}\left(\mu_{X, Y} \otimes \operatorname{id}_{Z}\right), \quad \mu_{X, \mathbb{I}}=\mu_{\mathbb{I}, X}=\mathrm{id}_{X}
$$

for all $X, Y, Z \in \mathrm{Ob} \mathcal{C}$.

Remark 4.4. Recall that naturality of $\mu_{X, Y}$ in $X, Y$ means that, given two morphisms $f: X \rightarrow X^{\prime}$ and $g: Y \rightarrow Y^{\prime}$ in $\mathcal{C}$, one has $(f \otimes g) \mu_{X, Y}=\mu_{X^{\prime}, Y^{\prime}}(f \otimes g)$.

Remark 4.5. The cocycle condition allows us to consider $\mu_{X, Y, Z} \in \operatorname{End}(X \otimes Y \otimes Z)$ which is defined as both $\mu_{X, Y} \otimes Z\left(\operatorname{id}_{X} \otimes \mu_{Y, Z}\right)$ and $\mu_{X \otimes Y, Z}\left(\mu_{X, Y} \otimes \operatorname{id}_{Z}\right)$. More generally, $\mu_{X_{1}, \ldots, X_{n}}, n \geq 3$, is defined recursively as $\mu_{X_{1}, \ldots, X_{n-2}, X_{n-1} \otimes X_{n}}\left(\mathrm{id}_{X_{1}} \otimes \ldots \otimes \mathrm{id}_{X_{n-2}} \otimes \mu_{X_{n-1}, X_{n}}\right)$ but has various other factorisations due to the cocycle condition.

Definition 4.6 (cocycle twist). Let $(A, m, \eta)$ be an algebra in $\mathcal{C}$ and $\mu$ be a normalised 2-cocycle on $\mathcal{C}$. Its twist by $\mu$ is defined to be the triple $\left(A, m \mu_{A, A}, \eta\right)$.

Theorem 4.7 (the cocycle twist functor). If $\mu$ is a normalised 2-cocycle on a monoidal category $\mathcal{C}$, the twist by $\mu$ of an algebra in $\mathcal{C}$ is again an algebra in $\mathcal{C}$. The twist by $\mu$ is a functor from $\mathcal{C}-\mathrm{Alg}$ to $\mathcal{C}-\mathrm{Alg}$ which is identity on morphisms.

Proof. Consider the twist $\left(A, m \mu_{A, A}, \eta\right)$ of $(A, m, \eta) \in \mathrm{Ob} \mathcal{C}$ - Alg. The morphism $m \mu_{A, A}$ is associative:

$$
\begin{aligned}
& m \mu_{A, A}\left(m \mu_{A, A} \otimes \operatorname{id}_{A}\right)=m\left(m \otimes \operatorname{id}_{A}\right) \mu_{A \otimes A, A}\left(\mu_{A, A} \otimes \operatorname{id}_{A}\right) \\
& =m\left(\mathrm{id}_{A} \otimes m\right) \mu_{A, A} \otimes A\left(\mathrm{id}_{A} \otimes \mu_{A, A}\right)=m \mu_{A, A}\left(\mathrm{id}_{A} \otimes m \mu_{A, A}\right),
\end{aligned}
$$

where the first and the third steps are by naturality of $\mu$, while the second step is by associativity of $m$ and the definition of cocycle. To show that $m \mu_{A, A}$ is unital with respect to $\eta$, use naturality of $\mu$ and unitality of $m$.

It remains to show that a morphism $f:\left(A, m_{A}, \eta_{A}\right) \rightarrow\left(B, m_{B}, \eta_{B}\right)$ in $\mathcal{C}$ - Alg remains a morphism between $\left(A, m_{A} \mu_{A, A}, \eta_{A}\right)$ and $\left(B, m_{B} \mu_{B, B}, \eta_{B}\right)$. We are given that $f m_{A}=m_{B}(f \otimes f)$ and want to prove that $f m_{A} \mu_{A, A}=m_{B} \mu_{B, B}(f \otimes f)$. But this clearly follows from naturality of $\mu$. Furthermore, we are given that $f \eta_{A}=\eta_{B}$, but this same property shows that $f$ intertwines the unit maps of the twisted algebras. 
Remark 4.8. Note that it is sometimes convenient to use the iterated product $m_{n}: A^{\otimes n} \rightarrow A$ for an algebra $A$, well-defined by associativity of $m$ : recursively, $m_{n}=m_{n-1}\left(\mathrm{id}_{A}^{\otimes n-2} \otimes m\right)$ for $n \geq 2$. We observe that the iterated product on the twist of $A$ by $\mu$ is equal to $m_{n} \mu_{A, A, \ldots, A}$ where the $n$-fold version $\mu_{A, A, \ldots, A}$ is defined in Remark 4.5 .

4.9. Coboundaries. The lazy cohomology of $\mathcal{C}$. Let us denote the collection of normalised 2-cocycles on a monoidal category $\mathcal{C}$ by $Z^{2}(\mathcal{C})$. Observe that natural automorphisms of the bifunctor $\otimes$ form a group Aut $\otimes$ under composition. (Warning: a group in this context may not be a set but is rather a class.)

We have $Z^{2}(\mathcal{C}) \subset$ Aut $\otimes$. We claim that $Z^{2}(\mathcal{C})$ is a subgroup of Aut $\otimes$. Indeed, observe that if $\mu, \nu: \otimes \rightarrow \otimes$ are natural transformations, then $\nu_{X, Y} \otimes Z$ and $\operatorname{id}_{X} \otimes \mu_{Y, Z}$ commute in $\operatorname{End}(X \otimes Y \otimes Z)$, precisely by naturality of $\nu$ in the second subscript. If $\mu, \nu \in Z^{2}(\mathcal{C})$, then

$$
\begin{aligned}
& \mu_{X, Y} \otimes Z^{\nu_{X, Y} \otimes Z}\left(\operatorname{id}_{X} \otimes \mu_{Y, Z} \nu_{Y, Z}\right)=\mu_{X, Y} \otimes Z\left(\operatorname{id}_{X} \otimes \mu_{Y, Z}\right) \nu_{X, Y} \otimes Z\left(\operatorname{id}_{X} \otimes \nu_{Y, Z}\right) \\
& =\mu_{X \otimes Y, Z}\left(\mu_{X, Y} \otimes \operatorname{id}_{Z}\right) \nu_{X \otimes Y, Z}\left(\mu_{X, Y} \otimes \operatorname{id}_{Z}\right) \\
& =\mu_{X \otimes Y, Z} \nu_{X \otimes Y, Z}\left(\mu_{X, Y} \nu_{X, Y} \otimes \operatorname{id}_{Z}\right),
\end{aligned}
$$

which shows that $Z^{2}(\mathcal{C})$ is closed under composition of natural transformations. Clearly $Z^{2}(\mathcal{C})$ is also closed under inverses, hence is a group, possibly non-abelian.

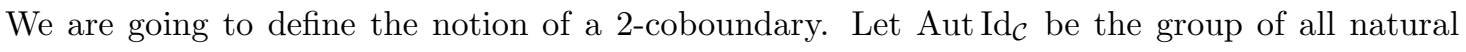
automorphisms of the identity functor of $\mathcal{C}$. We require all such automorphisms to be compatible with the associativity constraint of $\otimes$, to allow us to write $\phi_{X \otimes Y \otimes Z}$ for $\phi \in \operatorname{Aut} \mathcal{C}, X, Y, Z \in \mathrm{Ob} \mathcal{C}$. Define the map $d:$ Aut $\operatorname{Id}_{\mathcal{C}} \rightarrow$ Aut $\otimes$ by

$$
(d \phi)_{X, Y}=\phi_{X \otimes Y}^{-1}\left(\phi_{X} \otimes \phi_{Y}\right),
$$

and define the 2-coboundaries of $\mathcal{C}$ to be $B^{2}(\mathcal{C})=\operatorname{im} d \subset Z^{2}(\mathcal{C})$.

Proposition 4.10. $d$ is a group homomorphism, and $B^{2}(\mathcal{C})$ is a central subgroup of $Z^{2}(\mathcal{C})$.

Proof. Note that for any $\phi, \psi \in \operatorname{Aut}_{\mathcal{C}}, \psi_{X}^{-1} \otimes Y$ commutes with $\phi_{X} \otimes \phi_{Y}$ by naturality of $\psi$. This immediately implies that $d(\phi \psi)=(d \phi)(d \psi)$. Hence $d$ is a homomorphism, and $B^{2}(\mathcal{C})$ is a subgroup of Aut $\otimes$. Moreover, $d \phi \in Z^{2}(\mathcal{C})$ because

$$
\begin{aligned}
& (d \phi)_{X, Y \otimes Z}\left(\operatorname{id}_{X} \otimes(d \phi)_{Y, Z}\right)=\phi_{X \otimes Y \otimes Z}^{-1}\left(\phi_{X} \otimes \phi_{Y \otimes Z}\right)\left(\mathrm{id}_{X} \otimes \phi_{Y \otimes Z}^{-1}\left(\phi_{Y} \otimes \phi_{Z}\right)\right)
\end{aligned}
$$

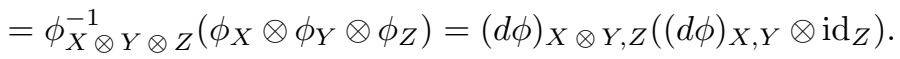

It remains to show that $d \phi$ commutes with $\mu \in Z^{2}(\mathcal{C})$. But $\phi_{X \otimes Y}^{-1}$ commutes with $\mu_{X, Y}$ by naturality of $\phi$, and $\phi_{X} \otimes \phi_{Y}$ commutes with $\mu_{X, Y}$ by naturality of $\mu$ in both $X$ and $Y$. 
Definition 4.11. The lazy cohomology group of a monoidal category $\mathcal{C}$ is the group

$$
H_{\ell}^{2}(\mathcal{C}):=Z^{2}(\mathcal{C}) / B^{2}(\mathcal{C})
$$

This notion, introduced by Panaite, Staic and Van Oystaeyen in [47, generalises the construction of the lazy cohomology group of a Hopf algebra, introduced by Bichon and Carnovale [10] based on a work [51] by Schauenburg. Currently there are not enough explicit calculations of this recently introduced analogue of the Schur multiplier, but see Bichon - Kassel 11] and references therein. If $G$ is a finite group, the lazy cohomology of the category of $G$-graded $\mathbb{C}$-vector spaces is $M(G)$, a finite abelian group. However, the monoidal category of $\mathbb{C} G$-modules is a natural object to be considered, and there are examples of finite groups $G$ for which $H_{\ell}^{2}(\mathbb{C} G$-modules $)$ is a non-abelian group.

Note that if two cocycles on $\mathcal{C}$ are in the same class in $H_{\ell}^{2}(\mathcal{C})$, the twist functors they induce on $\mathcal{C}$ - Alg are naturally isomorphic.

4.12. Example: the category $R-\mathcal{M}^{G}$. Let $R$ be a commutative ring and $G$ be a finite group. Denote by $R-\mathcal{M}^{G}$ the monoidal category of $R$-modules with $G$-grading. In other words (see Definition 3.5),

- objects of $R-\mathcal{M}^{G}$ are pairs $(V, \delta)$ where $V$ is an $R$-module and $\delta: V \rightarrow V \otimes_{R} R G$ is an $R$-module map;

- morphisms $f:\left(V, \delta_{V}\right) \rightarrow\left(W, \delta_{W}\right)$ are $R$-module maps $f: V \rightarrow W$ such that $\left(f \otimes \operatorname{id}_{R G}\right) \delta_{V}=$ $\delta_{W} f ;$

- the monoidal product of $\left(V, \delta_{V}\right)$ and $\left(W, \delta_{W}\right)$ is $V \otimes_{R} W$ where the coaction $\delta_{V} \otimes_{R} W$ is $\delta_{V} \otimes_{R} \delta_{W}$ followed by the group multiplication map $R G \otimes_{R} R G \rightarrow R G$;

- the unit object is $\mathbb{I}=R$ with coaction $\delta=\mathrm{id}_{R} \otimes_{R} 1_{G}$.

Then each normalised 2-cocycle $\mu \in Z^{2}\left(G, R^{\times}\right)$gives rise to a categorical cocycle on $R$ - $\mathcal{M}^{G}$ via

$$
\mu_{X, Y}=\sum_{g, h \in G} \mu(g, h)\left(\operatorname{id}_{X} \underset{R}{\otimes} \pi_{g} \underset{R}{\otimes} \operatorname{id}_{Y} \underset{R}{\otimes} \pi_{h}\right)\left(\delta_{X} \underset{R}{\otimes} \delta_{Y}\right) \quad \in \operatorname{End}_{R}(X \underset{R}{\otimes} Y),
$$

where $\pi_{g}: R G \rightarrow R$ is an $R$-module map defined by $\pi_{g}(g)=1, \pi_{g}(k)=0, g, k \in G, g \neq k$.

The category $R-\mathcal{M}^{G}$ - Alg is the category of $G$-graded algebras over $R$. It remains to note that the categorical twist of $A \in \mathrm{Ob} R-\mathcal{M}^{G}$ - Alg by $\mu_{X, Y}$ is the same as the twist $A_{\mu}$ considered in the previous section.

4.13. The cocycle extension functor for $\mathbb{C}$-algebras. The categorical setup accommodates the cocycle extension construction from 3.7, which will now be interpreted as a functor. We write $\mathcal{M}^{G}$ to denote $\mathbb{C}-\mathcal{M}^{G}$. 
Recall that if $\mu \in Z^{2}(G, \Gamma)$ where $\Gamma$ is an abelian group, and $A$ is an algebra over $\mathbb{C}$, the cocycle extension $\widetilde{A}_{\mu}$ of $A$ is the algebra $(\mathbb{C} \Gamma \otimes A)_{\mu}$ over $\mathbb{C} \Gamma$. Clearly, $\mathbb{C} \Gamma \otimes-$ is a faithful functor from $\mathcal{M}^{G}$ - Alg to $\mathbb{C} \Gamma-\mathcal{M}^{G}$ - Alg. Recall also the specialisation at $z=q$ which is a functor from $\mathbb{C} C_{m}-\mathcal{M}^{G}$ - Alg to $\mathcal{M}^{G}$ - Alg. We write this functor as $\left.(\cdot)\right|_{z=q}$. We summarise the so far developed categorical interpretation of extensions and twists of $\mathbb{C}$-algebras in the following

Theorem 4.14. Let $G$ be a finite group and $\Gamma$ be an abelian group.

1. To each cocycle $\mu \in Z^{2}(G, \Gamma)$ there corresponds a cocycle extension functor

$$
\widetilde{(\cdot)_{\mu}}: \mathcal{M}^{G}-\mathrm{Alg} \rightarrow \mathbb{C} \Gamma-\mathcal{M}^{G}-\mathrm{Alg}, \quad A \mapsto \widetilde{A_{\mu}} .
$$

The algebra $\widetilde{A}_{\mu}$ is a flat $\Gamma$-deformation of $A$.

2. If $\mu_{1}, \mu_{2}$ are cohomologous cocycles, the functors $\widetilde{(\cdot)_{\mu_{1}}}$ and $\widetilde{(\cdot)_{\mu_{2}}}$ are naturally isomorphic.

3. To each cocycle $\nu \in Z^{2}\left(G, \mathbb{C}^{\times}\right)$there corresponds a cocycle twist functor

$$
(\cdot)_{\nu}: \mathcal{M}^{G}-\mathrm{Alg} \rightarrow \mathcal{M}^{G}-\mathrm{Alg}, \quad A \mapsto A_{\nu} .
$$

4. For each $\nu \in Z^{2}\left(G, \mathbb{C}^{\times}\right)$one can find a finite cyclic group $C_{m}=\left\langle z \mid z^{m}=1\right\rangle$, an mth root of unity $q \in \mathbb{C}^{\times}$and a cocycle $\mu \in Z^{2}\left(G, C_{m}\right)$ such that there is a natural isomorphism

$$
\left.(\cdot)_{\nu} \cong(\cdot)\right|_{z=q} \circ \widetilde{(\cdot)_{\mu}}
$$

of functors.

\section{Cocycle twists of BRAidings And Nichols Algebras}

In this section, we work with a monoidal category with a braiding. If $\Psi$ is a braiding on an abelian monoidal category $\mathcal{C}$, to each object of $V$ of $\mathcal{C}$ there is associated an algebra $\mathcal{B}(V, \Psi)$ in $\mathcal{C}$. This is a general categorical version of Nichols algebra, a quantum group-theoretic construction which has become quite popular among Hopf algebra theorists. We introduce $\mathcal{B}(V, \Psi)$ in a category which is not necessarily $k$-linear for a field $k$; we use this construction in the category $R-{ }_{G} \mathcal{Y} \mathcal{D}^{G}$ of Yetter-Drinfeld modules for a group $G$ over a commutative ring $R$. One of the goals of this section is to show how $\mathcal{B}(V, \Psi)$ behaves under a cocycle twist.

5.1. Hopf algebras in a braided category. A braiding on a monoidal category $\mathcal{C}$ is a natural isomorphism $\Psi: \otimes \rightarrow \otimes^{\text {op }}$ that satisfies the hexagon condition. A monoidal category equipped with a braiding is a braided category. The standard reference is Joyal - Street [35], see also an expository paper [50] by Savage. The braiding yields, for each pair of objects $X, Y \in \mathrm{Ob} \mathcal{C}$, an isomorphism

$$
\Psi_{X, Y}: X \otimes Y \rightarrow Y \otimes X
$$

For example, the category $R$ - Mod has the trivial braiding, $\Psi=\tau$, defined by $\tau_{X, Y}\left(x \otimes_{R} y\right)=y \otimes_{R} x$. 
A braiding on a monoidal category $\mathcal{C}$ gives rise to a monoidal product on the category $\mathcal{C}$ - Alg. Namely, if $\left(A, m_{A}, \eta_{A}\right),\left(B, m_{B}, \eta_{B}\right) \in \mathrm{Ob}(\mathcal{C}-\mathrm{Alg})$, the object $A \otimes B$ becomes an algebra with respect to the multiplication map

$$
m_{A \otimes B}=\left(m_{A} \otimes m_{B}\right)\left(\operatorname{id}_{A} \otimes \Psi_{B, A} \otimes \operatorname{id}_{B}\right): A \otimes B \otimes A \otimes B \rightarrow A \otimes B
$$

and the unit map $\eta_{A} \otimes \eta_{B}$. The associativity of $m_{A \otimes B}$ follows from the hexagon condition and the naturality of $\Psi$. The unit object of $\mathcal{C}$ - Alg is $\left(\mathbb{I}, \mathbb{I} \otimes \mathbb{I} \stackrel{\sim}{\rightarrow} \mathbb{I}, \mathrm{id}_{\mathbb{I}}\right)$.

A bialgebra in a braided category $\mathcal{C}$ is an algebra $(B, m, \eta)$ in $\mathcal{C}$ equipped with two extra morphisms, the coproduct $\Delta: B \rightarrow B \otimes B$ which is coassociative in the usual sense and is a morphism of algebras, and $\epsilon: B \rightarrow \mathbb{I}$ satisfying the counit condition with respect to $\Delta$. Note that the braiding $\Psi$ is involved in the algebra structure on $B \otimes B$ and therefore affects the definition of $\Delta$. A Hopf algebra in $\mathcal{C}$ is a bialgebra equipped with a $\mathcal{C}$-morphism $S: B \rightarrow B$ satisfying the definition of an antipode. It is easy to observe that the trivial algebra $\mathbb{I}$ as above is also a Hopf algebra in $\mathcal{C}$.

An introduction to Hopf algebras in braided categories can be found in Majid [42, ch. 14].

5.2. Duals and rigidity. Half-adjoints. Kernels of a pairing. Let $X$ be an object in a monoidal category $\mathcal{C}$. A right dual of $X$ is an object $X^{*}$ together with two morphisms,

$$
\mathrm{ev}_{X}: X^{*} \otimes X \rightarrow \mathbb{I}, \quad \operatorname{coev}_{X}: \mathbb{I} \rightarrow X \otimes X^{*}
$$

which satisfy

$$
\begin{aligned}
\left(X \cong \mathbb{I} \otimes X \stackrel{\operatorname{coev}_{X} \otimes \operatorname{id}_{X}}{\longrightarrow} X \otimes X^{*} \otimes X \stackrel{\mathrm{id}_{X} \otimes \mathrm{ev}_{X}}{\longrightarrow} X\right) & =X \stackrel{\mathrm{id}_{X}}{\longrightarrow} X, \\
\left(X^{*} \cong X^{*} \otimes \mathbb{I} \stackrel{\mathrm{id}_{X^{*}} \otimes \operatorname{coev}_{X}}{\longrightarrow} X^{*} \otimes X \otimes X^{*} \stackrel{\mathrm{ev}_{X} \otimes \mathrm{id}_{X^{*}}}{\longrightarrow} X^{*}\right) & =X^{*} \stackrel{\mathrm{id}_{X^{*}}}{\longrightarrow} X^{*} .
\end{aligned}
$$

A right dual is unique up to an isomorphism. A left dual ${ }^{*} X$ of $X$ is a right dual of $X$ in the category $\left(\mathcal{C}, \otimes^{\text {op }}, \mathbb{I}\right)$. In particular, $X$ is a right dual of ${ }^{*} X$.

An object $X$ is rigid if it has right and left duals in $\mathcal{C}$. Rigid objects form a full subcategory of $\mathcal{C}$.

If $X$ and $Y$ are rigid objects in a monoidal category $\mathcal{C}$, each morphism $X \otimes Y \stackrel{f}{\rightarrow} Z$ has two half-adjoints:

$$
\begin{aligned}
& X \stackrel{f^{b}}{\longrightarrow} Z \otimes Y^{*} \quad=\quad\left(X \stackrel{\mathrm{id}_{X} \otimes \operatorname{coev}_{Y}}{\longrightarrow} X \otimes Y \otimes Y^{*} \stackrel{f \otimes \mathrm{id}_{Y^{*}}}{\longrightarrow} Z \otimes Y^{*}\right),
\end{aligned}
$$

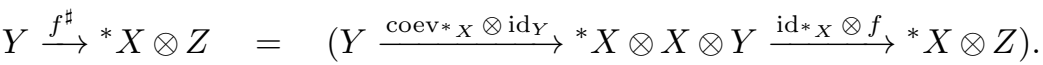

A pairing between two objects $X, Y$ in $\mathcal{C}$ is a morphism $\kappa: X \otimes Y \rightarrow \mathbb{I}$. For example, ev $_{X}$ is a pairing between $X^{*}$ and $X$ if $X$ is rigid. If $\mathcal{C}$ is additive, there is a zero pairing $X \otimes Y \stackrel{0}{\rightarrow} \mathbb{I}$ between any two objects. If the category $\mathcal{C}$ is abelian, we can define the right and left kernels of the pairing $\kappa$ by

$$
\operatorname{ker}_{l} \kappa=\operatorname{ker} \kappa^{b}, \quad \operatorname{ker}_{r} \kappa=\operatorname{ker} \kappa^{\sharp} .
$$


Note that a pairing $\kappa: X \otimes Y \rightarrow \mathbb{I}$ gives rise to a pairing between $X \otimes X$ and $Y \otimes Y$ :

$$
\kappa\left(\operatorname{id}_{X} \otimes \kappa \otimes \operatorname{id}_{Y}\right): X \otimes X \otimes Y \otimes Y \rightarrow \mathbb{I}
$$

that is, the rightmost copy of $X$ is paired with the leftmost copy of $Y$, and vice versa. In the same fashion $\kappa$ defines a pairing between $X^{\otimes n}$ and $Y^{\otimes n}$ by first acting on the innermost copy of $X \otimes Y$ in $X^{\otimes n} \otimes Y^{\otimes n}$.

5.3. Hopf duality pairing. Let $A, B$ be two bialgebras in a braided category $\mathcal{C}$. A duality pairing between $A$ and $B$ is a pairing $\kappa: A \otimes B \rightarrow \mathbb{I}$ such that

$$
\begin{array}{ll}
\kappa\left(\Delta_{A} \otimes \operatorname{id}_{B} \otimes \operatorname{id}_{B}\right)=\kappa\left(\operatorname{id}_{A} \otimes m_{B}\right): A \otimes B \otimes B \rightarrow \mathbb{I}, & \kappa\left(\eta_{A} \otimes \operatorname{id}_{B}\right)=\epsilon_{B}: B \rightarrow \mathbb{I}, \\
\kappa\left(\operatorname{id}_{A} \otimes \operatorname{id}_{A} \otimes \Delta_{B}\right)=\kappa\left(m_{A} \otimes \operatorname{id}_{B}\right): A \otimes A \otimes B \rightarrow \mathbb{I}, & \kappa\left(\operatorname{id}_{A} \otimes \eta_{B}\right)=\epsilon_{A}: A \rightarrow \mathbb{I} .
\end{array}
$$

If $A$ and $B$ are Hopf algebras, one also requires that $\kappa\left(S_{A} \otimes \operatorname{id}_{B}\right)=\kappa\left(\operatorname{id}_{A} \otimes S_{B}\right)$.

5.4. The free braided Hopf algebra. Now assume that the monoidal category $\mathcal{C}$ satisfies the conditions in 4.2 - that is, admits countable direct sums - and is additive. The free algebra $T(V)$ of an object $V$ of $\mathcal{C}$ has a canonical structure of a Hopf algebra in $\mathcal{C}$. Namely, let

$$
i_{n}: V^{\otimes n} \rightarrow T(V)
$$

denote the canonical injection. Consider

$$
d_{1}: V \stackrel{\sim}{\rightarrow} \mathbb{I} \otimes V \stackrel{i_{0} \otimes i_{1}}{\longrightarrow} T(V) \otimes T(V), \quad d_{2}: V \stackrel{\sim}{\rightarrow} V \otimes \mathbb{I} \stackrel{i_{1} \otimes i_{0}}{\longrightarrow} T(V) \otimes T(V) .
$$

Define $\left.\Delta\right|_{V}: V \rightarrow T(V) \otimes T(V)$ as $\left.\Delta\right|_{V}=d_{1}+d_{2}$. Also, define $\left.\Delta\right|_{\mathbb{I}}$ to be $\mathbb{I} \stackrel{\sim}{\rightarrow} \mathbb{I} \otimes \mathbb{I} \stackrel{i_{0} \otimes i_{0}}{\longrightarrow}$ $T(V) \otimes T(V)$. Following Majid [39], see also [11, ch. 10], one checks that there is a unique family of morphisms

$$
\Delta_{n}:=\left.\Delta\right|_{V^{\otimes n}}: V^{\otimes n} \rightarrow T(V), \quad n \geq 2,
$$

such that the resulting morphism $\Delta: T(V) \rightarrow T(V) \otimes T(V)$ is a morphism of algebras. The coassociativity of $\Delta$ then automatically follows from the coassociativity of $\Delta_{1}$.

The counit morphism $\epsilon$ on $T(V)$ is defined via $\left.\epsilon\right|_{\mathbb{I}}=\operatorname{id}_{\mathbb{I}}$ and $\left.\epsilon\right|_{V \otimes n}=0$ for $n \geq 1$. The antipode is $\left.S\right|_{V}=i_{1} \circ\left(-\mathrm{id}_{V}\right)$ extended to $T(V)$ as a braided antialgebra map. The details can be found in [41, ch. 10]; here is an explicit formula for the component $\Delta_{n}$ of the coproduct which is wholly in terms of the braiding $\Psi:=\Psi_{V, V}$ on $V$. Majid introduces the braided integers

$$
[n]_{\Psi}=\operatorname{id}_{V}^{\otimes n}+\Psi_{n-1, n}+\Psi_{n-1, n} \Psi_{n-2, n-1}+\ldots+\Psi_{n-1, n} \Psi_{n-2, n-1} \cdots \Psi_{1,2} \quad \in \text { End } V^{\otimes n}
$$


where the leg notation $\Psi_{i, i+1}$ stands for $\operatorname{id}_{V}^{\otimes i-1} \otimes \Psi \otimes \operatorname{id}_{V}^{\otimes n-i-1}$. In particular, $[1]_{\Psi}=\operatorname{id}_{V}$ and $[2]_{\Psi}=$ $\operatorname{id}_{V \otimes 2}+\Psi$. Furthermore, he defines the braided binomial coefficients $\left[\begin{array}{l}n \\ k\end{array}\right]_{\Psi} \in$ End $V^{\otimes n}$ recursively by

$$
\left[\begin{array}{l}
n \\
0
\end{array}\right]_{\Psi}=\operatorname{id}_{V \otimes n}, \quad\left[\begin{array}{c}
n-1 \\
n
\end{array}\right]_{\Psi}=0, \quad\left[\begin{array}{l}
n \\
k
\end{array}\right]_{\Psi}=\Psi_{k, k+1} \cdots \Psi_{n-1, n}\left(\left[\begin{array}{l}
n-1 \\
k-1
\end{array}\right]_{\Psi} \otimes \operatorname{id}_{V}\right)+\left[\begin{array}{c}
n-1 \\
k
\end{array}\right] \otimes \mathrm{id}_{V} .
$$

It is convenient to use the isomorphism $V^{\otimes n} \stackrel{\sim}{\rightarrow} V^{\otimes k} \otimes V^{\otimes n-k}$ to assume that

$$
\left[\begin{array}{l}
n \\
k
\end{array}\right]_{\Psi}: V^{\otimes n} \rightarrow V^{\otimes k} \otimes V^{\otimes n-k}
$$

which leads to

$$
\Delta_{n}=\sum_{k=0}^{n}\left(i_{k} \otimes i_{n-k}\right)\left[\begin{array}{l}
n \\
k
\end{array}\right]_{\Psi}: V^{\otimes n} \rightarrow T(V) \otimes T(V) .
$$

5.5. The duality pairing between $T(V)$ and $T\left(V^{*}\right)$. If $V$ is a rigid object of $\mathcal{C}$, there is a unique pairing $\kappa: T\left(V^{*}\right) \otimes T(V) \rightarrow \mathbb{I}$ such that

$$
\left.\kappa\right|_{V^{*} \otimes V}=\mathrm{ev}_{V}, \quad \kappa \text { is a Hopf duality pairing. }
$$

Necessarily the pairing between $V^{\otimes n}$ and $V^{* \otimes m}$ is 0 unless $n=m$. It is shown in 39] that $\kappa$ is given by

$$
\kappa_{n}:=\left.\kappa\right|_{V^{* \otimes n} \otimes V^{\otimes n}}=\mathrm{ev}_{V^{\otimes n}} \circ\left(\operatorname{id}_{V^{* \otimes n}} \otimes[n] !_{\Psi}\right)=\mathrm{ev}_{V^{\otimes n}} \circ\left([n] !_{\Psi^{*}} \otimes \mathrm{id}_{V^{\otimes n}}\right)
$$

where $\Psi=\Psi_{V, V}$ and $\Psi^{*}=\Psi_{V^{*}, V^{*}}$, which is necessarily the adjoint of $\Psi$ with respect to $\operatorname{ev}_{V \otimes V}$. The braided factorial is the endomorphism

$$
[n] !_{\Psi}=\left([1]_{\Psi} \otimes \operatorname{id}_{V \otimes n-1}\right)\left([2]_{\Psi} \otimes \operatorname{id}_{V \otimes n-2}\right) \cdots[n]_{\Psi}
$$

of $V^{\otimes n}$. The braided factorial is also known as the braided (Woronowicz) symmetriser of degree $n$. One can see explicitly that $\kappa$ is a Hopf duality pairing by observing that the braided binomial theorem holds [11, proof of Proposition 10.4.13]:

$$
\left([k] !_{\Psi} \otimes[n-k] !_{\Psi}\right)\left[\begin{array}{l}
n \\
k
\end{array}\right]_{\Psi}=[n] !_{\Psi}
$$

modulo the isomorphism $V^{\otimes k} \otimes V^{\otimes n-k} \stackrel{\sim}{\rightarrow} V^{\otimes n}$.

Remark 5.6 (braided symmetriser). We give the braided symmetriser $[n] !_{\Psi}$ as a product of braided integers, but its expansion (or, rather, expansions, as they depend on a choice of a reduced word for each element of $S_{n}$ ) is also useful. For the purposes of this remark only, write $\Psi_{i}$ to denote the endomorphism $\Psi_{i, i+1}$ of $V^{\otimes n}$. It follows from the hexagon axiom satisfied by $\Psi$ that the operators $\Psi_{1}, \ldots, \Psi_{n-1}$ satisfy the braid relations

$$
\Psi_{i} \Psi_{i+1} \Psi_{i}=\Psi_{i+1} \Psi_{i} \Psi_{i+1}, \quad 1 \leq i \leq n-2 ; \quad \Psi_{i} \Psi_{j}=\Psi_{j} \Psi_{i}, \quad j>i+1 .
$$


If $\sigma \in S_{n}$ is a permutation of $\{1, \ldots, n\}$, write $\sigma=s_{i_{1}} s_{i_{2}} \ldots s_{i_{l}}$ in a shortest possible way, where $s_{i}=(i, i+1)$. Put $\Psi_{\sigma}=\Psi_{i_{1}} \Psi_{i_{2}} \ldots \Psi_{i_{l}}$; this does not depend on the choice of the reduced (i.e., shortest) word $i_{1}, i_{2}, \ldots, i_{l}$ for $\sigma$ because of the braid relations satisfied by the $\Psi_{i}$. One then has

$$
[n] !_{\Psi}=\sum_{\sigma \in S_{n}} \Psi_{\sigma} \quad \in \quad \text { End } V^{\otimes n}
$$

5.7. The functor $\mathcal{B}$. Nichols algebras. We now come to an important class of Hopf algebras in a braided category $\mathcal{C}$. We have seen in 5.4 shows that if $\mathcal{C}$ is additive, to each $V \in \mathrm{Ob} \mathcal{C}$ there is associated a free braided Hopf algebra $T(V)$. If $V$ is rigid, there is a canonical Hopf duality pairing $T\left(V^{*}\right) \otimes T(V) \rightarrow \mathbb{I}$.

Now assume that $\mathcal{C}$ is an abelian braided category. The free braided Hopf algebra $T(V)$ of a rigid object $V$ is in general not a rigid object, but $V^{\otimes n}$ is. The half-adjoint of $\kappa_{n}$ is

$$
\kappa_{n}^{b}=[n] !_{\Psi}: V^{\otimes n} \rightarrow V^{\otimes n},
$$

where $\Psi=\Psi_{V, V}$. Consider the total Woronowicz symmetriser

$$
\operatorname{Wor}(\Psi)=\bigoplus_{n=1}^{\infty} i_{n} \kappa_{n}^{b}=\bigoplus_{n=1}^{\infty} i_{n}[n] !_{\Psi} \quad \in \quad \operatorname{End} T(V) .
$$

Following Majid's approach in [39, 41, one can quotient out the kernel of Wor $(\Psi)$ to kill the right kernel of the pairing $\kappa_{n}$ for all $n$. Denote

$$
\mathcal{B}(V, \Psi)=T(V) / \operatorname{ker} \operatorname{Wor}(\Psi) .
$$

This is an object in $\mathcal{C}$. Moreover, one can check that the product on a Hopf algebra $B$ in $\mathcal{C}$ induces a product on the quotient of $B$ modulo the (left or right) kernel of a Hopf duality pairing; same with coproduct. Therefore, $\mathcal{B}(V, \Psi)$ is a braided Hopf algebra.

Moreover, by naturality of the braiding $\Psi$, for each morphism $f: V \rightarrow W$ in $\mathcal{C}$ and for all $n$ one has $f^{\otimes n} \circ[n] !_{\Psi_{V, V}}=[n] !_{\Psi_{W, W}} \circ f^{\otimes n}$. This shows that the construction of $\mathcal{B}(V, \Psi)$ is functorial, that is, we are dealing with a functor

$$
\mathcal{B}(-, \Psi): \mathcal{C} \rightarrow \mathcal{C}-\mathrm{Alg}
$$

Note that although $\mathcal{C}$ - Alg is a monoidal category due to the braiding on $\mathcal{C}$, the functor $\mathcal{B}$ is not a monoidal functor.

In the setting where $\mathcal{C}$ is a $\mathbb{k}$-linear tensor category over some field $\mathbb{k}$ - that is, all objects are $\mathbb{k}$-vector spaces with some additional structure, and $\otimes$ is $\otimes_{\mathbb{k}}$ on underlying spaces - the $\mathbb{k}$-algebra $\mathcal{B}(V, \Psi)$ is known as the Nichols algebra of $V$, following Andruskiewitsch and Schneider [2].

Remark 5.8. To construct $\mathcal{B}(V, \Psi)$, the category $\mathcal{C}$ was assumed to be abelian, which guarantees the existence of quotient object; remember, $\mathcal{B}(V, \Psi)$ is the quotient of $T(V)$ by the kernel of $\oplus_{n}[n] !_{\Psi}$. 
However, if the category $\mathcal{C}$ is merely additive, the quotient of $T(V)$ by the said kernel may or may not exist in $\mathcal{C}$ for a given braiding $\Psi$. Sometimes we would like to work in an additive monoidal category which is not abelian: think of free $R$-modules with respect to $\otimes_{R}$ where $R$ is a commutative ring. The existence of $\mathcal{B}(V, \Psi)$ in such a category is not guaranteed a priori but will be proved by other means in special cases.

5.9. The cocycle twist of a braiding and of $\mathcal{B}(V, \Psi)$. Recall that a cocycle $\mu \in Z^{2}(\mathcal{C})$ is a natural automorphism of the monoidal product $\otimes$ on $\mathcal{C}$, and a braiding $\Psi$ is a natural transformation $\otimes \rightarrow \otimes^{\mathrm{op}}$. In an obvious way, $\mu^{\mathrm{op}}$ given by $\mu_{X, Y}^{\mathrm{op}}=\mu_{Y, X}$ is a natural automorphism of $\otimes^{\mathrm{op}}$. The following is then the way to twist $\Psi$ by $\mu$. It can be checked directly that the result is again a braiding (or see [47, Proposition 2.7]):

Lemma 5.10. $\Psi_{\mu}=\mu^{\mathrm{op}} \circ \Psi \circ \mu^{-1}$ is a braiding on $\mathcal{C}$.

Thus, the new braiding $\Psi_{\mu}$ is given by the formula

$$
\left(\Psi_{\mu}\right)_{X, Y}=\mu_{Y, X} \Psi_{X, Y} \mu_{X, Y}^{-1} \quad \text { for } X, Y \in \mathrm{Ob} \mathcal{C} .
$$

Let us find out the relationship between the algebras $\mathcal{B}(V, \Psi)$ and $\mathcal{B}\left(V, \Psi_{\mu}\right)$.

Theorem 5.11 (twisting of $\mathcal{B}(V, \Psi)$ ). Let $V$ be an object of an abelian monoidal category $\mathcal{C}$ which admits free algebras. Let $\Psi$ be a braiding on $\mathcal{C}$ and $\mu \in Z^{2}(\mathcal{C})$. Then

$$
\mathcal{B}\left(V, \Psi_{\mu}\right) \cong \mathcal{B}(V, \Psi)_{\mu}
$$

as algebras in $\mathcal{C}$, where $\mathcal{B}(V, \Psi)_{\mu}$ is the twist of $\mathcal{B}(V, \Psi)$ by the cocycle $\mu$.

Proof. Denote by $\mu_{n}$ the morphism $\mu_{V, V, \ldots, V}: V^{\otimes n} \rightarrow V^{\otimes n}$ introduced in Remark 4.5. For consistency, write $\mu_{0}=\mathrm{id}_{\mathbb{I}}$ and $\mu_{1}=\mathrm{id}_{V}$. Observe the following propety of $\mu_{n}, n \geq 3$, obtained by iterating the cocycle condition: $\mu_{V \otimes k} V^{\otimes n-k}\left(\mu_{k} \otimes \mu_{n-k}\right)=\mu_{n} i_{k}^{n}$, where $i_{k}^{n}$ denotes the canonical isomorphism between $V^{\otimes k} \otimes V^{\otimes n-k}$ and $V^{\otimes n}$.

It follows that the $\mathcal{C}$-morphism $\oplus_{n=0}^{\infty} i_{n} \mu_{n}: T(V) \rightarrow T(V)$ is in fact an isomorphism between the algebras $T(V)$ and $T(V)_{\mu}$.

Indeed, the product in $T(V)$ restricted to $V^{\otimes k} \otimes V^{\otimes n-k}$ coincides with $i_{k}^{n}$. The product on $T(V)_{\mu}$, which has the same underlying object $\oplus_{n=0}^{\infty} V^{\otimes n}$ as $T(V)$, is defined on $V^{\otimes k} \otimes V^{\otimes n-k}$ as $i_{k}^{n} \mu_{V \otimes k, V \otimes n-k}$. But then we have

$$
i_{k}^{n} \mu_{V^{\otimes k}, V^{\otimes n-k}}\left(\mu_{k} \otimes \mu_{n-k}\right)=\mu_{n} i_{k}^{n},
$$

which precisely says that $\oplus_{n=0}^{\infty} i_{n} \mu_{n}$ intertwines the two products on the object $T(V)$, the $T(V)$-product and the $T(V)_{\mu}$-product. 
To get the desired isomorphism between $\mathcal{B}(V, \Psi)$ and $\mathcal{B}\left(V, \Psi_{\mu}\right)$, we have to pass to the quotient algebras. It follows from the definition of $\Psi_{\mu}$ and of $[n] !_{\Psi}$ that

$$
[n] !_{\Psi_{\mu}}=\mu_{n}[n] !_{\Psi} \mu_{n}^{-1}
$$

which means that the isomorphism $\oplus_{n=0}^{\infty} i_{n} \mu_{n}$ between $T(V)$ and $T(V)_{\mu}$ induces an isomorphism between $\mathcal{B}\left(V, \Psi_{\mu}\right)=T(V) / \oplus_{n} \operatorname{ker}[n] !_{\Psi_{\mu}}$ and $\left(T(V) / \oplus_{n} \operatorname{ker}[n] !_{\Psi}\right)_{\mu}=\mathcal{B}(V, \Psi)_{\mu}$.

5.12. Twists of a braiding by monoidal automorphisms of the category. Let us point out that there is another way to twist a braiding $\Psi$ on a given monoidal category $\mathcal{C}$. Suppose that $F: \mathcal{C} \rightarrow \mathcal{C}$ is a monoidal functor which is strictly invertible; that is, an automorphism of $\mathcal{C}$ as a monoidal category. Define a new braiding $\Psi^{F}$ by

$$
\Psi_{X, Y}^{F}=F^{-1}\left(\Psi_{F(X), F(Y)}\right), \quad X, Y \in \mathrm{Ob} \mathcal{C} .
$$

It is straightforward to check that $\Psi^{F}$ is again a braiding on $\mathcal{C}$.

In one of the main examples below, a cocycle twist of a braiding coincides with a twist by an automorphism:

$$
\Psi_{\mu}=\Psi^{F}
$$

In this case, there is more we can say about the algebra $\mathcal{B}(V, \Psi)_{\mu}$. By Theorem $5.11, \mathcal{B}(V, \Psi)_{\mu} \cong$ $\mathcal{B}\left(V, \Psi_{\mu}\right)$. But clearly $F\left(\mathcal{B}\left(V, \Psi^{F}\right)\right)=\mathcal{B}(F(V), \Psi)$; here $F$ on the left-hand side is viewed as an automorphism of the category $\mathcal{C}$ - Alg. We conclude that

$$
F\left(\mathcal{B}(V, \Psi)_{\mu}\right)=\mathcal{B}(F(V), \Psi)
$$

This observation is used in the next section.

\section{Extensions And twists of Yetter-Drinfeld Modules}

We have described a general approach to algebras $\mathcal{B}(V, \Psi)$ and their cocycle twists that works in arbitrary braided monoidal categories. On the other hand, the mainstream research into Nichols algebras focuses on the braided category ${ }_{H} \mathcal{Y D}^{H}$ of Yetter-Drinfeld modules over a $\mathbb{k}$-linear Hopf algebra $H$, denoted ${ }_{G} \mathcal{Y} \mathcal{D}^{G}$ when $H=\mathbb{k} G$ is a group algebra. (There are four versions of the Yetter-Drinfeld module category: $\mathcal{Y} \mathcal{D}_{H}^{H},{ }_{H} \mathcal{Y} \mathcal{D}^{H},{ }^{H} \mathcal{Y} \mathcal{D}_{H},{ }_{H}^{H} \mathcal{Y D}$, but the differences between them are immaterial.)

We would like to get rid of the $\mathbb{k}$-linearity assumption. Let $R$ be a commutative ring, and consider Yetter-Drinfeld modules which are free $R$-modules - a mild generalisation of vector spaces - with an action and a coaction of a finite group $G$. This category is the appropriate target category for the cocycle extension functor for Yetter-Drinfeld modules, associated to $\mu \in Z^{2}\left(G, R^{\times}\right)$. This functor 
generalises the cocycle twist construction for Yetter-Drinfeld modules, originally due to Majid and Oeckl [44, now widely used in the literature on Nichols algebras — see for example [1].

6.1. The monoidal category ${ }_{R, G} \mathcal{Y} \mathcal{D}^{G}$. Let $R$ be a commutative ring and $G$ be a finite group. The category ${ }_{R, G} \mathcal{Y} \mathcal{D}^{G}$ of $R$-free Yetter-Drinfeld modules for $R G$ consists of $G$-graded $R G$-modules

$$
Y=\bigoplus_{g \in G} Y_{g}
$$

where each $V_{g}$ is a free $R$-module. The action $\triangleright$ of $G$ and the grading are compatible in the sense

$$
g \triangleright Y_{h}=Y_{g h g^{-1}}
$$

for all $g, h \in G$.

Recall that a $G$-grading on $Y$ can be written as a coaction $\delta: Y \rightarrow Y \otimes_{R} R G$, see Definition 3.5. For coactions we will use the Sweedler sigma notation [56, but without the sigma. Such notation is often found in modern Hopf algebra literature, see for example [42]. We write

$$
\delta(v)=v^{(0)} \underset{R}{\otimes} v^{(1)} \in Y \otimes R G,
$$

where the summation is implicit. The Yetter-Drinfeld module condition for $Y$ is then written as

$$
\begin{aligned}
\delta(h \triangleright v) & =\left(h \triangleright v^{(0)}\right) \underset{R}{\otimes} h v^{(1)} h^{-1} \\
& =h \triangleright \delta(v) \quad \forall h \in G, \forall v \in Y .
\end{aligned}
$$

The last equality assumes that $Y \otimes_{R} R G$ is viewed as the tensor product of $R G$-modules $Y$ and $(R G)_{\mathrm{ad}}$.

The tensor product $Y \otimes_{R} Z$ of two Yetter-Drinfeld modules $Y, Z$ is again a Yetter-Drinfeld module, with coaction given by $\delta\left(y \otimes_{R} z\right)=\left(y^{(0)} \otimes_{R} z^{(0)}\right) \otimes_{R} y^{(1)} z^{(1)}$. Note that the tensor product, over $R$, of two free $R$-modules is again a free $R$-module. The category ${ }_{R, G} \mathcal{Y D}^{G}$ is thus a monoidal category.

We do not compute the lazy cohomology $H_{\ell}^{2}\left(R, G \mathcal{Y} \mathcal{D}^{G}\right)$ but observe that each cocycle $\mu \in$ $Z^{2}\left(G, R^{\times}\right)$gives rise to a categorical cocycle on ${ }_{R, G} \mathcal{Y} \mathcal{D}^{G}$.

Lemma 6.2. A group cocycle $\mu \in Z^{2}\left(G, R^{\times}\right)$gives rise to a categorical cocycle on ${ }_{R, G} \mathcal{Y D}^{G}$ defined as follows: $\mu_{X, Y} \in \operatorname{End}\left(X \otimes_{R} Y\right)$ acts on $X_{g} \otimes_{R} Y_{h}$ by $\mu(g, h) \in R^{\times}$, where $g, h \in G$.

Proof. Clearly $\mu_{X, Y}$ is natural in both $X$ and $Y$ and is an automorphism of $X \otimes_{R} Y$ (commutes with the $G$-action, preserves the $G$-grading). The categorical cocycle condition for $\mu_{X, Y}$ is the same as the cocycle condition for $\mu(g, h)$. 
6.3. ${ }_{R, G} \mathcal{Y} \mathcal{D}^{G}$ as a braided category. The category ${ }_{R, G} \mathcal{Y} \mathcal{D}^{G}$ is braided: the standard braiding is given by

$$
\Psi_{Y, Z}(y \underset{R}{\otimes} z)=(g \triangleright z) \underset{R}{\otimes} y \quad \text { for } y \in Y_{g}, z \in Z .
$$

Yetter-Drinfeld modules which are free $R$-modules of finite rank are rigid objects in ${ }_{R, G} \mathcal{Y} \mathcal{D}^{G}$. The dual object to $Y$ is $Y^{*}=\operatorname{Hom}(Y, R)$ as an $R G$-module, with grading $\left(Y^{*}\right)_{g}=\left(Y_{g^{-1}}\right)^{*}$.

Example 6.4 (Modules with trivial $G$-grading). Any $R G$-module $V$ is a Yetter-Drinfeld module with respect to the trivial grading, $\delta(v)=v \otimes_{R} 1$ for $v \in V$. This provides an embedding of the category of $R$-free $R G$-modules as a full subcategory in ${ }_{R, G} \mathcal{Y} \mathcal{D}^{G}$.

Example 6.5 (The adjoint module $(R G)_{\text {ad }}$ ). Let $(R G)_{\text {ad }}$ denote the free $R$-module $R G$ where the group $G$ acts by conjugation. We refer to $(R G)_{\text {ad }}$ as the adjoint module for $G$. Observe that $(R G)_{\mathrm{ad}}$ is a Yetter-Drinfeld module for $G$ with respect to the grading given by $(R G)_{g}=R g$ for $g \in G$. This grading yields the coaction $\delta(g)=g \otimes_{R} g$.

Example 6.6 (The Yetter-Drinfeld module $V \otimes_{R} R G$ ). Take the tensor product of the above two examples: to each $G$-module $V$ there is associated a Yetter-Drinfeld module $V \otimes_{R} R G$ with $G$-action $h \triangleright\left(v \otimes_{R} g\right)=(h \triangleright v) \otimes_{R} h g h^{-1}$ and coaction $\delta\left(v \otimes_{R} g\right)=v \otimes_{R} g \otimes_{R} g$. Here $v \in V$ and $g, h \in G$.

Various modifications of the construction of $V \otimes_{R} R G$ provide a supply of useful Yetter-Drinfeld modules. For instance, if $C \subset G$ is a conjugacy class, $V \otimes_{R} R C$ is clearly a Yetter-Drinfeld submodule of $V \otimes_{R} R G$ in ${ }_{R, G} \mathcal{Y} \mathcal{D}^{G}$. When $R=\mathbb{k}$ is a field, this is an important example of a rack with a cocycle as in [1].

6.7. Nichols algebras of Yetter-Drinfeld modules. As we mentioned earlier, if $R=\mathbb{k}$ is a field, the category ${ }_{R, G} \mathcal{Y} \mathcal{D}^{G}$ is one of the most typical examples of braided categories used to construct Nichols algebras $\mathcal{B}(Y, \Psi)$. If $Y$ is a Yetter-Drinfeld module, we will often write $\mathcal{B}(Y)$ to denote $\mathcal{B}(Y, \Psi)$ when $\Psi$ is the standard braiding given in 6.3 . We work with $\mathbb{k}=\mathbb{C}$ and denote the category $\mathbb{C},{ }_{G} \mathcal{Y} \mathcal{D}^{G}$ simply by ${ }_{G} \mathcal{Y} \mathcal{D}^{G}$. Recall from Section 5 that the Nichols algebra of $Y \in \mathrm{Ob}_{G} \mathcal{Y} \mathcal{D}^{G}$ is

$$
\mathcal{B}(Y)=T(Y) / \oplus_{n} \operatorname{ker}[n] !_{\Psi}, \quad \Psi=\Psi_{Y, Y}
$$

For general $R$, however, it is not clear why the algebra $\mathcal{B}(Y)$ exists in the category ${ }_{R, G} \mathcal{Y} \mathcal{D}^{G}$, because this category may not be abelian. It exists if the quotient object exists in the category; the algebra structure is then automatic. We leave the following question open:

Question 6.8. Let $Y$ be a Yetter-Drinfeld module, over a commutative ring $R$, for a group $G$. Assume that $Y$ is free as $R$-module. Does the algebra $\mathcal{B}(Y)$ exist in the category of free $R$-modules? 
We obtain some positive evidence related to Question 6.8 showing that if $R$ is a $\mathbb{C}$-algebra, then cocycle extensions of $\mathcal{B}(Y)$ where $Y \in \mathrm{Ob}{ }_{G} \mathcal{Y D}^{G}$ are Nichols algebras of certain extensions of $Y$ in the category $R, G \mathcal{Y D}^{G}$. Note that a cocycle extension of a $\mathbb{C}$-algebra is automatically free as $R$-module.

The rest of this section is devoted to extending a Nichols algebra of $Y \in \mathrm{Ob}_{G} \mathcal{Y} \mathcal{D}^{G}$ by a cocycle $\mu \in Z^{2}\left(G, R^{\times}\right)$, where $R$ is a commutative $\mathbb{C}$-algebra. The result turns out to be an algebra $\mathcal{B}\left(\widetilde{Y}_{\mu}\right)$ of a certain object $\widetilde{Y}_{\mu}$ of ${ }_{R, G} \mathcal{Y} \mathcal{D}^{G}$. According to the scheme outlined in 3.7, 5.11] and 5.12, the extension is carried out in three steps.

6.9. Step 1: extension by the trivial cocycle (extension of scalars). Let $Y \in \mathrm{Ob}_{G} \mathcal{Y D}^{G}$. As any $\mathbb{C}$-algebra, $\mathcal{B}(Y)$ has trivial extension $R \otimes_{\mathbb{C}} \mathcal{B}(Y)$ which is an algebra over $R$. The next Lemma shows that this $R$-algebra is $\mathcal{B}\left(R \otimes_{\mathbb{C}} Y\right)$. That is, the extension of scalars functor commutes with the functor $\mathcal{B}$.

Lemma 6.10 (extension of scalars for Yetter-Drinfeld modules). Let $R$ be $a \mathbb{C}$-algebra and $Y \in$ Ob ${ }_{G} \mathcal{Y} \mathcal{D}^{G}$. Let the $R$-module $\widetilde{Y}=R \otimes_{\mathbb{C}} Y$ be the extension of scalars of $Y$, with the action and grading extended from $Y$ by

$$
g \triangleright(r \underset{R}{\otimes} y)=r \underset{R}{\otimes}(g \triangleright y), \quad r \in R ; \quad \tilde{Y}=\bigoplus_{g \in G} R \underset{\mathbb{C}}{\otimes} Y_{g} .
$$

Then $\tilde{Y} \in \mathrm{Ob}_{R, G} \mathcal{Y} \mathcal{D}^{G}$. Moreover, $R \otimes_{\mathbb{C}} \mathcal{B}(Y)=\mathcal{B}(\widetilde{Y})$.

Proof. By construction, $\widetilde{Y}=R \otimes_{\mathbb{C}} Y$ is a free $R$-module. It is easy to see that the Yetter-Drinfeld condition for the action and grading on $R \otimes_{\mathbb{C}} Y$ follows from the Yetter-Drinfeld condition on $Y$.

Denote by $T_{R}:{ }_{R, G} \mathcal{Y} \mathcal{D}^{G} \rightarrow{ }_{R, G} \mathcal{Y} \mathcal{D}^{G}$ - Alg the free algebra functor in the category ${ }_{R, G} \mathcal{Y D}^{G}$. It is clear, e.g., by considering the basis of the free $R$-module $(\widetilde{Y})^{\otimes_{R}^{n}}$, that $T_{R}(\widetilde{Y})=R \otimes_{\mathbb{C}} T(Y)$. Let $\Psi=$ $\Psi_{Y, Y} \in \operatorname{End}_{\mathbb{C}}(Y \otimes Y), \widetilde{\Psi}=\Psi_{\widetilde{Y}, \widetilde{Y}} \in \operatorname{End}_{R}\left(\widetilde{Y} \otimes_{R} \widetilde{Y}\right)$ be the braidings on $Y$ and on $\widetilde{Y}$. The definition of a braiding on a Yetter-Drinfeld module implies that $\widetilde{\Psi} \in \operatorname{End}_{R}\left(\widetilde{Y} \otimes_{R} \tilde{Y}\right)=\operatorname{End}_{R}\left(R \otimes_{\mathbb{C}} Y \otimes_{\mathbb{C}} Y\right)$ is the same as $\operatorname{id}_{R} \otimes_{\mathbb{C}} \Psi$. It follows that the braided factorials $[n] !_{\widetilde{\Psi}}$ are also expressed $\operatorname{as~}_{R} \otimes_{\mathbb{C}}[n] !_{\Psi}$. Because $R \otimes_{\mathbb{C}}(-)$ is an exact functor from $\mathbb{C}$-vector spaces to $R$-modules, $\operatorname{ker}[n] !_{\widetilde{\Psi}}=R \otimes_{\mathbb{C}} \operatorname{ker}[n] !_{\Psi}$ and

$$
\mathcal{B}(\widetilde{Y})=(R \underset{\mathbb{C}}{\otimes} T(Y)) /\left(R \underset{\mathbb{C}}{\otimes} \operatorname{ker}[n] !_{\Psi}\right)=R \underset{\mathbb{C}}{\otimes}\left(T(Y) / \operatorname{ker}[n] !_{\Psi}\right)=R \underset{\mathbb{C}}{\otimes} \mathcal{B}(Y)
$$

6.11. Step 2: twisting $\mathcal{B}(\widetilde{Y})$ by $\mu \in Z^{2}\left(G, R^{\times}\right)$. Let now $Z \in \mathrm{Ob}_{R, G} \mathcal{Y} \mathcal{D}^{G}$ and $\mu \in Z^{2}\left(G, R^{\times}\right)$. We know how to twist $\mathcal{B}(Z)$ by a categorical cocycle, and Lemma 6.2 explains how to interpret $\mu$ as a categorical cocycle. Applying Theorem 5.11 and the formula for $\Psi_{\mu}$ from Lemma 5.10, we obtain 
Corollary 6.12. The twist of $\mathcal{B}(Z)$ by $\mu$ is the algebra $\mathcal{B}\left(Z, \Psi_{\mu}\right)$ in ${ }_{R, G} \mathcal{Y} \mathcal{D}^{G}$. The braiding $\Psi_{\mu}$ on the category ${ }_{R, G} \mathcal{Y D}^{G}$ is given by the formula

$$
\left(\Psi_{\mu}\right)_{X, Y}(x \underset{R}{\otimes} y)=\mu\left(g h g^{-1}, g\right) \mu(g, h)^{-1}(g \triangleright y) \underset{R}{\otimes} x, \quad x \in X_{g}, y \in Y_{h}, g, h \in G .
$$

6.13. Step 3: realisation of $\Psi_{\mu}$ via an automorphism of the Yetter-Drinfeld category. Finally, we observe that $\Psi_{\mu}$, given in Corollary 6.12, can be realised as $\Psi^{F}$ where $F:{ }_{R, G} \mathcal{Y D}^{G} \rightarrow$ ${ }_{R, G} \mathcal{Y} \mathcal{D}^{G}$ is a strictly invertible functor. Indeed, the formula for $\left(\Psi_{\mu}\right)_{X, Y}$ shows that this is a standard braiding between $F(X)$ and $F(Y)$ where the functor $F=F_{\mu}$ is defined in

Lemma 6.14. Let $\mu \in Z^{2}\left(G, R^{\times}\right)$. There exists a functor $F_{\mu}:{ }_{R, G} \mathcal{Y} \mathcal{D}^{G} \rightarrow{ }_{R, G} \mathcal{Y} \mathcal{D}^{G}$ such that

- $F_{\mu}(X)$ is $X$ as an $R$-module and $G$-comodule;

- if $\triangleright: G \times X \rightarrow X$ is the $G$-action, then the $G$-action on $F(X)$ is given by $g \triangleright_{\chi} x=\chi(g, k)(g \triangleright x)$, $g, k \in G, x \in X_{k}$;

$-F_{\mu}$ is identity on morphisms. Here

$$
\chi(g, k)=\mu\left(g k g^{-1}, g\right) \mu(g, k)^{-1} \in R^{\times} \quad \text { for } g, k \in G .
$$

The functor $F_{\mu}$ is strictly invertible with inverse $F_{\mu^{-1}}$.

Remark 6.15. The functor $F_{\mu}$ arises from the Majid-Oeckl construction [44, Theorem 2.7], in a special case when a Hopf algebra $H$ is the group algebra. Strictly speaking, in 44 the construction is over a field, while our setup allows us to carry it over in ${ }_{R, G} \mathcal{Y} \mathcal{D}^{G}$. The formula for $\chi$ in terms of $\mu$ is an adaptation of the Hopf algebra formula from [4] to the case of a group algebra of $G$. Note that we have already seen the function $\chi$ in 2.8 in the case $G=S_{n}$.

Moreover, in the linear case $(R=\mathbb{k}$ is a field), the extension construction for Nichols algebras becomes a twist functor $(\cdot)_{\mu}:{ }_{G} \mathcal{Y} \mathcal{D}^{G}-\mathrm{Alg} \rightarrow{ }_{G} \mathcal{Y D}^{G}-\mathrm{Alg}$. The construction can be carried out for a Nichols algebra associated to a rack with cocycle. In that setting, $\mu$ is a cocycle that arises from the cohomology of a rack - this is similar to the cohomology of a finite group $G$ which in degree 2 yields the Schur multiplier $M(G)$. See for example [1]. The formula for $\chi$ in the form given in Lemma 6.14 is the same as [1, equation (20)] and apparently goes back to [25].

Proof of Lemma 6.14. It is enough to show that $F_{\mu}$ is well defined, all axioms of a functor being fulfilled automatically. We need to check that the formula for $\triangleright_{\chi}$ defines an action of $G$. The condition to be checked is $\chi\left(g, h k h^{-1}\right) \chi(h, k)=\chi(g h, k)$. This is the same as $\frac{\chi(g h, k)}{\chi(g, h \triangleright \text { ad } k)}=\chi(h, k)$ for all $g, h, k \in G$. (We write $\frac{z}{t}$ to denote $z t^{-1}$ where $z, t \in R^{\times}$.) Rewrite the left-hand side in terms of $\mu$ and use the cocycle equation for $\mu$ :

$$
\frac{\mu(g h, k)}{\mu\left((g h) \triangleright_{\mathrm{ad}} k, g h\right)} \cdot \frac{\mu\left((g h) \triangleright_{\mathrm{ad}} k, g\right)}{\mu\left(g, h \triangleright_{\mathrm{ad}} k\right)}=\frac{\mu(g h, k) \mu(g, h)}{\mu\left(g h k h^{-1}, h\right) \mu\left(g, h k h^{-1}\right)}=\frac{\mu(g, h k) \mu(h, k)}{\mu\left(g, h k h^{-1} h\right) \mu\left(h k h^{-1}, h\right)}
$$


which is $\chi(h, k)$ as required. It remains to observe that morphisms $X \rightarrow Y$ compatible with the $G$-action $\triangleright$ and the $G$-grading are also compatible with $\triangleright_{\chi}$.

Remark 6.16. We repeat an observation made in 2.8, the condition on $\chi$, checked in the proof of the Lemma, can be interpreted to read that $\chi$ is a 1-cocycle on $G$ with coefficients in $\operatorname{Fun}\left(G, R^{\times}\right)$ viewed as a right module for $G$ with non-trivial action.

The above implies the following

Corollary 6.17. Let $Y \in \mathrm{Ob}{ }_{G} \mathcal{Y} \mathcal{D}^{G}, R$ be a commutative $\mathbb{C}$-algebra and $\mu \in Z^{2}\left(G, R^{\times}\right)$. Let $\chi=\chi_{\mu}$ be as in Lemma 6.14. Define $\widetilde{Y}_{\mu} \in \mathrm{Ob}_{R, G} \mathcal{Y D}^{G}$ to be $R \otimes_{\mathbb{C}} Y$ as R-module with G-action $\triangleright_{\chi}$ and $G$-grading naturally extended from $Y$. Then

$$
\widetilde{\mathcal{B}(Y)_{\mu}}=\mathcal{B}\left(\widetilde{Y}_{\mu}\right)
$$

as objects in the category ${ }_{R, G} \mathcal{Y} \mathcal{D}^{G}-\mathrm{Alg}$.

6.18. Fomin-Kirillov algebras, Majid algebras, and cocycles. We finish this section by considering an explicit example of a cocycle extension of a Nichols algebra. Let $S_{n}$ be the symmetric group of degree $n$. Denote by $X_{n}$ the conjugacy class of all transpositions in $S_{n}$.

Suppose that $q: S_{n} \times X_{n} \rightarrow \mathbb{C}^{\times}$is a function satisfying

$$
q(\rho \sigma, \tau)=q\left(\rho, \sigma \tau \sigma^{-1}\right) q(\sigma, \tau), \quad \forall \rho, \sigma \in S_{n}, \forall \tau \in X_{n} .
$$

This means that $\sigma \mapsto q(\sigma,-)$ is a normalised 1-cocycle in $Z^{1}\left(S_{n}, F u n\left(X_{n}, \mathbb{C}^{\times}\right)\right)$. Clearly, $q$ is determined by its values $q(\sigma, \tau)$ where $\sigma, \tau \in X_{n}$, and these values must satisfy the rack cocycle condition [1]. Then one can define a Yetter-Drinfeld module structure on the vector space

$$
\mathbb{C} X_{n}=\operatorname{span}\left\{e_{(i j)} \mid(i j) \in X_{n}\right\}
$$

as follows. The basis vector $e_{\tau} \in \mathbb{C} X_{n}$ has $S_{n}$-degree $\tau$; this gives the $S_{n}$-grading on $\mathbb{C} X_{n}$. The action of $S_{n}$ on $\mathbb{C} X_{n}$ is via

$$
\sigma \triangleright_{q} e_{\tau}=q(\sigma, \tau) e_{\sigma \tau \sigma^{-1}}
$$

It is easy to see that the Yetter-Drinfeld condition holds; denote the resulting Yetter-Drinfeld module for $\mathbb{C} S_{n}$ by $\left(X_{n}, q\right)$. One can consider the Nichols algebra $\mathcal{B}\left(X_{n}, q\right)$ which is an algebra in ${ }_{S_{n}} \mathcal{Y} \mathcal{D}^{S_{n}}$.

Two particular examples of $\mathcal{B}\left(X_{n}, q\right)$ arose in the literature. First, consider the function defined for $\sigma \in X_{n}$ and $i<j$ by

$$
q_{1}(\sigma,(i j))= \begin{cases}1, & \text { if } \sigma(i)<\sigma(j) \\ -1, & \text { if } \sigma(i)>\sigma(j)\end{cases}
$$


The Fomin-Kirillov algebra $\mathcal{E}_{n}$, introduced in [26] as a noncommutative model for the Schubert calculus, can be defined by taking the generators and the quadratic relations of $\mathcal{B}\left(X_{n}, q_{1}\right)$. Conjecturally, $\mathcal{B}\left(X_{n}, q_{1}\right)$ is a quadratic algebra and hence coincides with $\mathcal{E}_{n}$. The same Nichols algebra $\mathcal{B}\left(X_{n}, q_{1}\right)$ was defined by Milinski and Schneider in 45. It was shown by Fomin and Kirillov [26, section 5] that the elements

$$
\theta_{j}=-\sum_{1 \leq i<j} e_{(i j)}+\sum_{j<i \leq n} e_{(j i)}, \quad j=1,2, \ldots, n,
$$

commute pairwise and generate a subalgebra of $\mathcal{E}_{n}$ isomorphic to the cohomology ring of the complex flag manifold $F l_{n}$. In [5], the same was proved for the Nichols algebra $\mathcal{B}\left(X_{n}, q_{1}\right)$. See [58] for a recent brief survey on the Fomin-Kirillov algebras.

The second example we would like to mention is

$$
q_{-1}(\sigma,(i j))=-1, \quad \forall \sigma, \tau \in X_{n},
$$

which, too, yields an element of $Z^{1}\left(S_{n}, F u n\left(X_{n}, \mathbb{C}^{\times}\right)\right)$and leads to a Nichols algebra $\mathcal{B}\left(X_{n}, q_{-1}\right)$. This Nichols algebra and its quadratic cover $\Lambda_{n}$ were considered by Majid in [43]. Conjecturally, $\Lambda_{n}=\mathcal{B}\left(X_{n}, q_{-1}\right)$. Majid showed that the elements

$$
\alpha_{j}=-\sum_{1 \leq i<j} e_{(i j)}-\sum_{j<i \leq n} e_{(j i)}=-\sum_{i \neq j} e_{(i j)}, \quad j=1,2, \ldots, n,
$$

pairwise anticommute in $\Lambda_{n}$ and generate a subalgebra of $\Lambda_{n}$ termed "the subalgebra of flat connections".

In fact, $q_{1}$ and $q_{-1}$ are the only elements of $Z^{1}\left(S_{n}, \operatorname{Fun}\left(X_{n}, \mathbb{C}^{\times}\right)\right)$for which the corresponding Nichols algebra generated by $X_{n}$ has a chance to be finite-dimensional, see [59]. It is known that $\mathcal{B}\left(X_{n}, q_{1}\right)$ and $\mathcal{B}\left(X_{n}, q_{-1}\right)$ are finite-dimensional for $n \leq 5$.

It was shown by Vendramin in [59] that the Nichols algebras $\mathcal{B}\left(X_{n}, q_{1}\right)$ and $\mathcal{B}\left(X_{n}, q_{-1}\right)$ in ${ }_{S_{n}} \mathcal{Y D}^{S_{n}}$ are cocycle twists of each other. We can describe the relevant cocycle $\mu \in Z^{2}\left(S_{n}, \mathbb{C}^{\times}\right)$as

$$
\mu=\left.[1, z]\right|_{z=-1}=[1,-1],
$$

where $[1, z] \in Z^{2}\left(S_{n}, C_{2}\right)$ is as defined in 2.5

6.19. The Nichols $\mathbb{C} C_{2}$-algebra over $S_{n}$. To finish this Section, we point out that both Nichols algebras $\mathcal{B}\left(X_{n}, q_{ \pm 1}\right)$ are specialisations of an algebra over $R:=\mathbb{C} C_{2}=\mathbb{C}[z] /\left\langle z^{2}-1\right\rangle$, obtained by the cocycle extension construction. There is a cocycle $q_{z} \in Z^{1}\left(S_{n}, F u n\left(X_{n}, R^{\times}\right)\right)$such that for $\sigma \in X_{n}$ and $i<j$,

$$
q_{z}(\sigma,(i j))= \begin{cases}z & \text { if } \sigma(i)<\sigma(j) \\ -1, & \text { if } \sigma(i)>\sigma(j)\end{cases}
$$


Consider $R X_{n}$ as an $R S_{n}$-module with the action

$$
\sigma \triangleright e_{(i j)}=q_{z}(\sigma,(i j)) e_{(\sigma(i) \sigma(j))} .
$$

This, together with the standard $S_{n}$-grading, makes $R X_{n}$ an object of ${ }_{R, S_{n}} \mathcal{Y} \mathcal{D}^{S_{n}}$ with the braiding given by

$$
\Psi_{z}\left(e_{\tau} \underset{R}{\otimes} e_{v}\right)=q_{z}(\tau, v) e_{\tau v \tau^{-1}} \underset{R}{\otimes} e_{\tau}, \quad \tau, v \in X_{n},
$$

and leads to the algebra

$$
\mathcal{B}\left(R X_{n}, q_{z}\right)=T\left(R X_{n}\right) / \operatorname{ker} \operatorname{Wor}\left(\Psi_{z}\right) \quad \in \mathrm{Ob}\left({ }_{R, S_{n}} \mathcal{Y} \mathcal{D}^{S_{n}}-\mathrm{Alg}\right)
$$

The theory of cocycle extensions developed so far leads to the following

Theorem 6.20. Let $R=\mathbb{C} C_{2}$ as above.

1. The algebra $\mathcal{B}\left(R X_{n}, q_{z}\right)$ is the cocycle extension of $\mathcal{B}\left(X_{n}, q_{1}\right)$ by the cocycle $\mu_{(1, z)} \in Z^{2}\left(S_{n}, C_{2}\right)$, and is also the cocycle extension of $\mathcal{B}\left(X_{n}, q_{-1}\right)$ by the cocycle $\mu_{(1, z)}\left(\left.\mu_{(1, z)}\right|_{z=-1}\right) \in Z^{2}\left(S_{n}, R^{\times}\right)$.

2. $\mathcal{B}\left(R X_{n}, q_{z}\right)$ is a $C_{2}$-flat deformation of both $\mathcal{B}\left(X_{n}, q_{1}\right)$ and $\mathcal{B}\left(X_{n}, q_{-1}\right)$, in particulaaaaaaaaaar, a free $R$-module. One has

$$
\left.\mathcal{B}\left(R X_{n}, q_{z}\right)\right|_{z=1}=\mathcal{B}\left(X_{n}, q_{1}\right),\left.\quad \mathcal{B}\left(R X_{n}, q_{z}\right)\right|_{z=-1}=\mathcal{B}\left(X_{n}, q_{-1}\right),
$$

hence

$$
\mathcal{B}\left(R X_{n}, q_{z}\right) \cong \frac{1+z}{2} \mathcal{B}\left(X_{n}, q_{1}\right) \oplus \frac{1-z}{2} \mathcal{B}\left(X_{n}, q_{-1}\right),
$$

as direct sum of two-sided ideals.

Note that the algebra $\mathcal{B}\left(R X_{n}, q_{z}\right)$ is generated, as a $\mathbb{C}$-algebra, by $z, e_{(i j)}, 1 \leq i<j \leq n$. The quadratic relations in $\mathcal{B}\left(R X_{n}, q_{z}\right)$ extend the relations in both $\mathcal{E}_{n}$ and $\Lambda_{n}$ : they are

$$
\begin{gathered}
z \text { is central, } \quad z^{2}=1, \\
e_{(i j)}^{2}=0, \quad e_{(i j)} e_{(s t)}=z e_{(s t)} e_{(i j)} \quad \text { if }\{i, j\} \cap\{s, t\}=\varnothing, \\
e_{(i j)} e_{(j k)}-z e_{(j k)} e_{(i k)}-z e_{(i k)} e_{(i j)}=0, \quad \text { if } i<j<k .
\end{gathered}
$$

\section{BRAIDED DOUBLES}

We will now review the braided doubles, introduced in [6] and [7]. Although much of the setup carries over to a general abelian monoidal category, we work with braided doubles over $\mathbb{C}$. This section contains general facts and constructions which will be needed later. In this Section, unadorned tensor products $\otimes$ always mean $\otimes_{\mathbb{C}}$. 
7.1. Deformations of semidirect products. Let $\triangleright$ be a left action of a group $G$ on an algebra $A$. The semidirect product of $A$ and $G$, denoted $A \rtimes G$, is an algebra with underlying vector space $A \otimes \mathbb{C} G$. The embeddings of $A$ and $\mathbb{C} G$ in $A \ltimes G$ via the maps $a \mapsto a \otimes 1$ and $g \mapsto 1 \otimes g$ are algebra morphisms, and the relation

$$
g a=(g \triangleright a) g
$$

holds in $A \rtimes G$ for all $g \in G, a \in A$. These conditions determine the multiplication in $A \rtimes G$ uniquely.

In particular, if $V$ is a $G$-module, the group $G$ acts on the free tensor algebra $T(V)=\bigoplus_{n=0}^{\infty} V^{\otimes n}$ by algebra automorphisms. Let

$$
R_{0} \subset T^{>0}(V)=\bigoplus_{n>0} V^{\otimes n}
$$

be a graded $G$-submodule of $T(V)$. The two-sided ideal $I_{0}$ of $T(V)$ generated by $R_{0}$ is $G$-invariant, and there is an algebra

$$
A_{0}=\left(T(V) / I_{0}\right) \rtimes G=\mathbb{C} G \oplus\left(V / V \cap R_{0}\right) \rtimes G \oplus \ldots,
$$

with a grading where the degree of $G$ is 0 and the degree of $V$ is 1 . We say that a subspace

$$
R \subset R_{0} \oplus \mathbb{C} G \subset T(V) \rtimes G
$$

is a deformation of $R_{0}$ if the projection $R_{0} \oplus \mathbb{C} G \rightarrow R_{0}$ restricts to a one-to-one linear map $R \stackrel{\sim}{\rightarrow} R_{0}$. In this situation, let $I$ be the ideal of $T(V) \rtimes G$ generated by $R$. The algebra

$$
A=(T(V) \rtimes G) / I
$$

is viewed as a deformation of $A_{0}$. Observe that putting $G$ in degree zero and $V$ in degree one defines an ascending filtration on $A$. In general, there is a surjective map

$$
A_{0} \rightarrow \operatorname{gr} A,
$$

where gr $A$ denotes the associated graded algebra of $A$ with respect to this filtration. The algebra $A$ is said to be a flat deformation of $A_{0}$, if this map is an isomorphism.

One is interested in the following problem: find deformations $R \subset R_{0} \oplus \mathbb{C} G$ of a given $R_{0} \subset$ $T^{>0}(V)$ such that $A$ is a flat deformation of $A_{0}$.

Example 7.2 (Degenerate affine Hecke algebras and symplectic reflection algebras). In the case where $R_{0}=\bigwedge^{2} V$, i.e., the span of $\{x \otimes y-y \otimes x \mid x, y \in V\}$, the above deformation problem was studied by Drinfeld in [20]. Here $A_{0}=S(V) \rtimes G$ where $S(V)=T(V) /\left\langle\bigwedge^{2} V\right\rangle$ is the algebra of symmetric tensors on $V$ (a free commutative algebra generated by $V$ ). Flat deformations $A$ of $A_{0}$ are referred to in 20] as degenerate affine Hecke algebras. If $V$ is a symplectic vector space and 
the action of a finite group $G$ preserves the symplectic form on $V$, such algebras $A$ are symplectic reflection algebras introduced by Etingof and Ginzburg in [24].

7.3. Braided doubles over a group $G$. Braided doubles were introduced by the authors in [6] for a general bialgebra $H$ in place of the group algebra $\mathbb{C} G$. Braided doubles are a class of solutions to the deformation problem considered in 7.1 in a special case where the space $V$ is "split".

Namely, let $V^{-}, V^{+}$be finite-dimensional $G$-modules, $V=V^{-} \oplus V^{+}$. We view $T\left(V^{ \pm}\right)$as graded subalgebras of $T(V)$. Let a graded space $R_{0}$ of relations in $T^{>0}(V)$ be of the form

$$
R_{0}=R^{-}+R^{+}+\operatorname{span}\left\{f \otimes v-v \otimes f \mid f \in V^{+}, v \in V^{-}\right\},
$$

where $R^{-} \subset T^{>0}\left(V^{-}\right)$and $R^{+} \subset T^{>0}\left(V^{+}\right)$are graded subspaces in $T^{>0}(V)$. Let

$$
A_{0}\left(R^{-}, R^{+}\right)=\left(T(V) /<R_{0}>\right) \rtimes G \text {. }
$$

Then it is not difficult to observe the following isomorphism of vector spaces:

$$
A_{0}\left(R^{-}, R^{+}\right) \cong T\left(V^{-}\right) /<R^{-}>\otimes \mathbb{C} G \otimes T\left(V^{+}\right) /<R^{+}>.
$$

That is, the algebra $A_{0}\left(R^{-}, R^{+}\right)$has three subalgebras $U^{ \pm} \cong T\left(V^{ \pm}\right) /<R^{ \pm}>, U^{0} \cong \mathbb{C} G$ generated by $V^{ \pm}$and $G$, respectively, and the multiplication map of $A_{0}\left(R^{-}, R^{+}\right)$yields a vector space isomorphism $U^{-} \otimes U^{0} \otimes U^{+} \stackrel{\sim}{\rightarrow} A_{0}\left(R^{-}, R^{+}\right)$. The "straightening" relations between $U^{0}$ and $U^{ \pm}$that allow us to write any element of $A_{0}\left(R^{-}, R^{+}\right)$as a linear combination of products $u^{-} u^{0} u^{+}$with $u^{i} \in U^{i}$ are:

- the semidirect product relations $g v=(g \triangleright v) g, f g=g\left(g^{-1} \triangleright f\right)$ for $g \in G, v \in V^{-}, f \in V^{+}$;

— the commutation relation $f v-v f=0$.

Braided doubles are obtained from $A_{0}\left(R^{-}, R^{+}\right)$by deforming the latter commutation relation: zero on the right is replaced by a linear combination of elements of the group $G$, with coefficients which depend linearly on $f$ and $v$. Formally, the deformation parameter is a linear map

$$
\beta: V^{+} \otimes V^{-} \rightarrow \mathbb{C} G,
$$

and the following algebra is a deformation of $A_{0}\left(R^{-}, R^{+}\right)$:

$$
A_{\beta}\left(R^{-}, R^{+}\right)=\frac{T\left(V^{-} \oplus V^{+}\right) \rtimes G}{<R^{-}, R^{+},\left\{f \otimes v-v \otimes f-\beta(f \otimes v) \mid f \in V^{+}, v \in V^{-}\right\}>} .
$$

Definition 7.4. The algebra $A_{\beta}\left(R^{-}, R^{+}\right)$is called a braided double over $G$, if it is a flat deformation of $A_{0}\left(R^{-}, R^{+}\right)$.

Note that $A_{\beta}\left(R^{-}, R^{+}\right)$coincides with $A_{0}\left(R^{-}, R^{+}\right)$when $\beta=0$, justifying the chosen notation. Furthermore, note that the linear map

$$
T\left(V^{-}\right) /<R^{-}>\otimes \mathbb{C} G \otimes T\left(V^{+}\right) /<R^{+}>\rightarrow A_{\beta}\left(R^{-}, R^{+}\right),
$$


given by the multiplication in $A_{\beta}\left(R^{-}, R^{+}\right)$, is surjective for any $\beta$. Flatness of the deformation means that this map is one-to-one.

It follows that, whenever $A_{\beta}\left(R^{-}, R^{+}\right)$is a braided double, the algebras $T\left(V^{-}\right) /<R^{-}>, \mathbb{C} G$ and $T\left(V^{+}\right) /<R^{+}>$are embedded in $A_{\beta}\left(R^{-}, R^{+}\right)$as subalgebras - as are, in fact, $\left(T\left(V^{-}\right) /<R^{-}>\right) \rtimes G$ and $G \ltimes\left(T\left(V^{+}\right) /<R^{+}>\right)$. For this reason, we say that a braided double $A_{\beta}\left(R^{-}, R^{+}\right)$has triangular decomposition over $\mathbb{C} G$.

Example 7.5. A standard example of a braided double is the quantum universal enveloping algebra $U_{q}(\mathfrak{g})$ where $\mathfrak{g}$ is a semisimple complex Lie algebra. The triangular decomposition of $U_{q}(\mathfrak{g})$ is $U_{q}^{-} \otimes \mathbb{C} K \otimes U_{q}^{+}$with $K$ a free Abelian group of rank $l=$ rk $\mathfrak{g}$ and $U_{q}^{ \pm}$quotients of free tensor algebras of rank $l$ by the quantum Serre relations, see [37.

Another example, of prime importance to the present paper, is the rational Cherednik algebras $H_{t, c}(G)$ of a finite complex reflection group $G \subset \mathrm{GL}(V)$. Here the triangular decomposition is of the form $S(V) \otimes \mathbb{C} G \otimes S\left(V^{*}\right)$, see [24].

The classical universal enveloping algebra $U(\mathfrak{g})$ of a semisimple Lie algebra $\mathfrak{g}$ is also a braided double, but over a commutative and cocommutative Hopf algebra $U(\mathfrak{h})$ rather than over a group (so not of the type we consider in the present paper). Here $\mathfrak{h}$ is a Cartan subalgebra of $\mathfrak{g}$. A choice of a Borel subalgebra of $\mathfrak{g}$ containing $\mathfrak{h}$ leads to the direct sum decomposition $\mathfrak{g}=\mathfrak{n}^{-} \oplus \mathfrak{h} \oplus \mathfrak{n}^{+}$, where $\mathfrak{n}^{-}$, respectively $\mathfrak{n}^{+}$, is spanned by negative, respectively positive, root vectors. This gives rise to the triangular decomposition $U(\mathfrak{g}) \cong U\left(\mathfrak{n}^{-}\right) \otimes U(\mathfrak{h}) \otimes U\left(\mathfrak{n}^{+}\right)$where $U\left(\mathfrak{n}^{+}\right)$is generated by simple root vectors modulo the Serre relations, see [55].

7.6. Hierarchy of braided doubles. Suppose that the $G$-modules $V^{-}$and $V^{+}$are fixed. The algebra $A_{\beta}\left(R^{-}, R^{+}\right)$depends on the triple $\left(\beta, R^{-}, R^{+}\right)$of parameters, where $\beta \in \operatorname{Hom}_{\mathbb{C}}\left(V^{+} \otimes V^{-}, \mathbb{C} G\right)$ and $R^{ \pm}$is a graded $G$-submodule of $T^{>0}\left(V^{ \pm}\right)$. One is interested in the class of such triples for which $A_{\beta}\left(R^{-}, R^{+}\right)$is a braided double. Clearly, this algebra does not change if $R^{-}$is replaced by the $G$-invariant two-sided ideal $I^{-}$of $T\left(V^{-}\right)$generated by $R^{-}$; same for $R^{+}$.

Definition 7.7. Recall that the $G$-module $(\mathbb{C} G)_{\text {ad }}$ (the adjoint module for $G$ ) is the group algebra $\mathbb{C} G$ where $G$ acts by conjugation, $g \triangleright_{\text {ad }} h=g h g^{-1}$. We say that a linear map $\beta: V^{+} \otimes V^{-} \rightarrow \mathbb{C} G$ is $G$-equivariant, if $\beta$ is a $G$-module map $V^{+} \otimes V^{-} \rightarrow(\mathbb{C} G)_{\text {ad }}$.

Basic facts about braided doubles, listed in the next theorem, were proved in [6]. We do not reproduce their proof here but explain, see Remark 7.9 below, how to remove the assumption $V^{+}=\left(V^{-}\right)^{*}$ made in [6]. We denote by $[a, b]$ the commutator $a b-b a$ in a given associative algebra $A$, and extend this notation to subspaces $U, V$ of $A$, writing $[U, V]=\operatorname{span}\{[u, v] \mid u \in U, v \in V\}$. 
Theorem 7.8. Let $G$ be a group and $V^{-}, V^{+}$be finite-dimensional $G$-modules. Let $\beta$ denote a linear map $V^{+} \otimes V^{-} \rightarrow \mathbb{C} G$, and $I^{ \pm}$denote proper graded ideals of $T\left(V^{ \pm}\right)$.

1. $A_{\beta}(0,0)$ is a braided double, if and only if $\beta$ is $G$-equivariant.

2. Let $\beta$ be $G$-equivariant. Then $A_{\beta}\left(I^{-}, I^{+}\right)$is a braided double iff $I^{ \pm}$are $G$-invariant and

$$
\left[V^{+}, I^{-}\right] \subset I^{-} \otimes \mathbb{C} G, \quad\left[I^{+}, V^{-}\right] \subset \mathbb{C} G \otimes I^{+} \quad \text { in the algebra } A_{\beta}(0,0) .
$$

3. The sum $I_{\beta}^{-}$of all ideals $I^{-} \subset T^{>0}\left(V^{-}\right)$that satisfy 2. also satisfies 2., same for $I_{\beta}^{+}$. For any braided double $A_{\beta}\left(I^{-}, I^{+}\right)$there are surjective algebra homomorphisms

$$
A_{\beta}(0,0) \rightarrow A_{\beta}\left(I^{-}, I^{+}\right) \rightarrow A_{\beta}\left(I_{\beta}^{-}, I_{\beta}^{+}\right) .
$$

4. The group $G$ acts on any braided double $A_{\beta}\left(I^{-}, I^{+}\right)$. As a $G$-module, $A_{\beta}\left(I^{-}, I^{+}\right)$is the same as the tensor product $T\left(V^{-}\right) / I^{-} \otimes(\mathbb{C} G)_{\text {ad }} \otimes T\left(V^{+}\right) / I^{+}$given by the triangular decomposition.

Remark 7.9. Both [6] and [7] define braided doubles as in 7.3 above but with additional restriction $V^{+}=\left(V^{-}\right)^{*}$. It might seem that braided doubles considered in the present paper are more general than in [6, 7, but in fact they are not. Indeed, let $V^{-}, V^{+}$be two finite-dimensional $G$-modules, and let $\beta: V^{+} \otimes V^{-} \rightarrow \mathbb{C} G$ be a linear map. Define a new $G$-module $U=V^{-} \oplus\left(V^{+}\right)^{*}$. The dual of $U$ is $U^{*}=V^{+} \oplus\left(V^{-}\right)^{*}$. One has

$$
U^{*} \otimes U=\left(V^{+} \otimes V^{-}\right) \oplus\left(V^{+} \otimes V^{+^{*}}\right) \oplus\left(V^{-*} \otimes V^{-}\right) \oplus\left(V^{-*} \otimes V^{+^{*}}\right),
$$

a direct sum of $G$-modules. Define

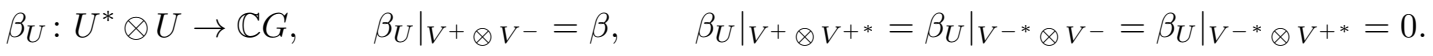

Then $\beta_{U}$ is a $G$-equivariant map if and only if $\beta$ is.

Now suppose that $\beta$ and $\beta_{U}$ are $G$-equivariant. In the algebra $A_{\beta_{U}}(0,0)$ which has triangular decomposition $T(U) \otimes \mathbb{C} G \otimes T\left(U^{*}\right)$, the subspace $V^{+*}$ of $U$ commutes with $U^{*}$, due to the way $\beta_{U}$ is defined. Similarly, $V^{-^{*}} \subset U^{*}$ commutes with $U$. By [7, Proposition 1.6], $A_{\beta_{U}}\left(V^{\left.+^{*}, V^{-*}\right)}\right.$ is a braided double. But $A_{\beta_{U}}\left(V^{+^{*}}, V^{-^{*}}\right) \cong T(U) /<V^{+^{*}}>\otimes \mathbb{C} G \otimes T\left(U^{*}\right) /<V^{-^{*}}>$ is exactly the algebra $A_{\beta}(0,0) \cong T\left(V^{-}\right) \otimes \mathbb{C} G \otimes T\left(V^{+}\right)$. Moreover, braided doubles $A_{\beta}\left(I^{-}, I^{+}\right)$as defined in 7.3 coincide with braided doubles of the form $A_{\beta_{U}}\left(I^{-}+\left\langle V^{-*}\right\rangle, I^{+}+\left\langle V^{+^{*}}\right\rangle\right)$, introduced in [6] and [7].

7.10. Morphisms of braided doubles. If $A$ and $B$ are braided doubles over the same group $G$, it makes sense to consider algebra maps between them which preserve the braided double structure. A morphism between $A$ and $B$ will mean a $G$-module algebra map

$$
A \cong T\left(V^{-}\right) / I^{-} \otimes \mathbb{C} G \otimes T\left(V^{+}\right) / I^{+} \stackrel{f}{\rightarrow} B \cong T\left(W^{-}\right) / J^{-} \otimes \mathbb{C} G \otimes T\left(W^{+}\right) / J^{+},
$$


such that

$$
f\left(V^{-}\right) \subseteq W^{-}, \quad f\left(V^{+}\right) \subseteq W^{+},\left.\quad f\right|_{\mathbb{C} G}=\operatorname{id}_{\mathbb{C} G} .
$$

Note that the restrictions of $f$ to $V^{-}$and to $V^{+}$determine the morphism $f$ uniquely. Also note that, pedantically, the above condition should be written as $f\left(V^{-} /\left(V^{-} \cap I^{-}\right)\right) \subseteq W^{-} /\left(W^{-} \cap J^{-}\right)$etc, because $V^{-}$may not be a subspace of $A$ if the graded ideal $I^{-}$of $T\left(V^{-}\right)$has a non-zero component in degree 1.

7.11. Minimal doubles. Let $V^{-}, V^{+}$be two finite-dimensional modules over a group $G$, and let $\beta: V^{+} \otimes V^{-} \rightarrow(\mathbb{C} G)_{\text {ad }}$ be a $G$-module map. By part 3 of Theorem 7.8 , there are largest possible ideals $I_{\beta}^{ \pm}$of $T^{>0}\left(V^{ \pm}\right)$such that $A_{\beta}\left(I_{\beta}^{-}, I_{\beta}^{+}\right)$is a braided double.

Definition 7.12. The algebra

$$
\bar{A}_{\beta}:=A_{\beta}\left(I_{\beta}^{-}, I_{\beta}^{+}\right) \cong T\left(V^{-}\right) / I_{\beta}^{-} \otimes \mathbb{C} G \otimes T\left(V^{+}\right) / I_{\beta}^{+}
$$

is called the minimal braided double associated to $\beta$.

Note that $\bar{A}_{\beta}$ is a quotient of every braided double with given $V^{ \pm}$and $\beta$. In general, the ideals

$I_{\beta}^{ \pm}$of relations in $\bar{A}_{\beta}$ can only be described implicitly as kernels of certain linear maps $[6$, Theorem 4.11]. For example, in degree 1 the relations are the left and right kernels of the bilinear map $\beta$ :

$$
I_{\beta}^{-} \cap V^{-}=\left\{v \in V^{-}: \beta(f \otimes v)=0 \forall f \in V^{+}\right\}, \quad I_{\beta}^{+} \cap V^{+}=\left\{f \in V^{+}: \beta(f \otimes v)=0 \forall v \in V^{-}\right\} .
$$

7.13. Braided doubles in the category of free $R$-modules. We finish this Section by observing that the definition of a braided double and some of the properties of braided doubles may carry over to monoidal categories more general than the $\mathbb{C}$-vector spaces Vect.

Let $R$ be a commutative ring. Consider the abelian monoidal category $\left(R\right.$ - Mod, $\left.\otimes_{R}, R\right)$ of $R$-modules. This category admits free algebras $T_{R}(V)$ of $V \in \mathrm{Ob}(R$-Mod). If $V$ is a free $R$-module, then so is

$$
T_{R}(V)=R \oplus V \oplus(V \underset{R}{\otimes} V) \oplus \cdots
$$

Let $G$ be a finite group and $V^{-}, V^{+}$be $R G$-modules which are free of finite rank over $R$. Suppose that $\beta: V^{+} \otimes_{R} V^{-} \rightarrow R G_{\text {ad }}$ is a $G$-equivariant map. Then the free $R$-module

$$
A_{\beta}(0,0)=T_{R}\left(V^{-}\right) \underset{R}{\otimes} R \underset{R}{\otimes} T_{R}\left(V^{+}\right)
$$

has an $R$-algebra structure of a free braided double over $R G$. The multiplication on $A_{\beta}(0,0)$ is defined in the same way as for $R=\mathbb{C}$, because one can repeat the construction from $[6$, Theorem 3.3], using the fact that $T_{R}\left(V^{ \pm}\right)$have bases over $R$. 
To construct general braided doubles over $R$, assume that $I^{ \pm} \subset T_{R}\left(V^{ \pm}\right)$are $R G$-invariant ideals such that $T_{R}\left(V^{ \pm}\right) / I^{ \pm}$are free $R$-modules. Then the following generalisation of part 2 of Theorem 7.8 holds. If

$$
\left[V^{+}, I^{-}\right] \subset I^{-} \underset{R}{\otimes} R G, \quad\left[I^{+}, V^{-}\right] \subset R G \underset{R}{\otimes} I^{+} \quad \text { in the algebra } A_{\beta}(0,0),
$$

then the $R$-algebra $A_{\beta}\left(I^{-}, I^{+}\right):=A_{\beta}(0,0) /<I^{-}, I^{+}>$has triangular decomposition $T_{R}\left(V^{-}\right) / I^{-} \otimes_{R}$ $R G \otimes_{R} T_{R}\left(V^{+}\right) / I^{+}$. This algebra $A_{\beta}\left(I^{-}, I^{+}\right)$is then called a braided double in the category of free $R$-modules.

We will be especially interested in quadratic doubles. In the case $R=\mathbb{C}$, they were studied in [7. From the above we deduce the following

Proposition 7.14. Let $V^{-}, V^{+}$be $R G$-modules which are free $R$-modules of finite rank, and let $\beta: V^{+} \otimes_{R} V^{-} \rightarrow R G_{\text {ad }}$ be a G-equivariant map. Let $R^{-} \subset V^{-} \otimes_{R} V^{-}, R^{+} \subset V^{+} \otimes_{R} V^{+}$be submodules of quadratic relations such that

- $T_{R}\left(V^{-}\right) /<R^{-}>$and $T_{R}\left(V^{+}\right) /<R^{+}>$are free $R$-modules;

- $V^{+}$commutes with $R^{-}$and $R^{+}$commutes with $V^{-}$in the free braided double $A_{\beta}(0,0)$.

Then $A_{\beta}\left(R^{-}, R^{+}\right)$is a braided double in the category of free $R$-modules.

Finally, as elsewhere in the present paper, our primary example of $R$ is $\mathbb{C} \Gamma$ where $\Gamma$ is an abelian group. We have the following monoidal category:

$$
(\Gamma-\text { Vect }, \otimes, \mathbb{I}):=(\text { free } \mathbb{C} \Gamma \text {-modules, } \underset{\mathbb{C} \Gamma}{\otimes}, \mathbb{C} \Gamma)
$$

Let $\widetilde{G}$ be a group containing $\Gamma$ as a central subgroup. Then $\mathbb{C} \widetilde{G}$ is an algebra over $\mathbb{C} \Gamma$, hence an algebra in $\Gamma$-Vect. There will be braided doubles in $\Gamma$ - Vect with triangular decomposition of the form $A \cong U^{-} \otimes_{\mathbb{C} \Gamma} \mathbb{C} \widetilde{G} \otimes_{\mathbb{C} \Gamma} U^{+}$. Morphisms between braided doubles in $\Gamma$ - Vect are defined in the same way as in 7.10 .

Because $\Gamma$ - Vect is an additive category which is not abelian in general, a quotient of an algebra in $\Gamma$ - Vect by a two-sided ideal does not always exist in the category. For this reason, we do not claim the existence of a minimal double in $\Gamma$ - Vect corresponding any given $\beta$.

Observe that any braided double in $\Gamma$-Vect, being free as a $\mathbb{C} \Gamma$-module, can be specialised to a braided double in Vect: the quotient of $A$ modulo $\mathbb{C} \Gamma_{+} A$ has triangular decomposition over $\mathbb{C} G$ where $G=\widetilde{G} / \Gamma$. In particular, $A$ is always a $\Gamma$-flat deformation of $A / \mathbb{C} \Gamma_{+} A$ in the sense of 3.8 . When $\Gamma=C_{k}=\left\langle z \mid z^{k}=1\right\rangle$, one has

$$
\left.\left.\left.A\right|_{z=1} \cong U^{-}\right|_{z=1} \underset{\mathbb{C}}{\otimes} \mathbb{C} G \underset{\mathbb{C}}{\otimes} U^{+}\right|_{z=1}, \quad G=\left.\widetilde{G}\right|_{z=1}
$$




\section{Braided Heisenberg doubles ANd BRAided Weyl algebras}

Among all braided doubles over a group $G$, there is a distinguished class of braided Heisenberg doubles, which correspond to Yetter-Drinfeld modules for $G$. We review their construction. A new result in this section is that a braided Heisenberg double is isomorphic to a semidirect product of $G$ with a braided Weyl algebra.

8.1. Braided Heisenberg doubles. Let $Y$ be a finite-dimensional Yetter-Drinfeld module $Y$ for $G$. Put $Y^{-}=Y$ and $Y^{+}=Y^{*}$. For $f \in Y^{*}, v \in Y$ define

$$
\beta(f \otimes v)=\left\langle f, v^{(0)}\right\rangle v^{(1)},
$$

where $\langle$,$\rangle is the G$-invariant pairing between $Y^{*}$ and $Y$, and $\delta(v)=v^{(0)} \otimes v^{(11)}$ is the $G$-coaction. Then $\beta$ is a $G$-equivariant map because of the Yetter-Drinfeld condition, see 6.1] Therefore, there is the minimal double

$$
\mathcal{H}_{Y}:=\bar{A}_{\beta}
$$

called a braided Heisenberg double [6, Section 5]. The defining relations in $\mathcal{H}_{Y}$ are given in terms of the braiding $\Psi$ on $Y$. Identify $Y^{*} \otimes Y^{*}$ with the dual space of $Y \otimes Y$ via the pairing

$$
\langle e \otimes f, v \otimes w\rangle=\langle f, v\rangle\langle e, w\rangle
$$

as in 5.2. By [6, Theorem 5.4], one has

$$
I_{\beta}^{-}=I_{\Psi}, \quad I_{\beta}^{+}=I_{\tau \Psi^{*} \tau},
$$

where $I_{\Psi}$ denotes the kernel of the Woronowicz symmetrisers as in Section [5, and $\tau$ is the transposition map on $Y^{*} \otimes Y^{*}$. Observe that $\Psi^{*}$ denotes the adjoint of $\Psi$ with respect to the above pairing and is the braiding on the Yetter-Drinfeld module $Y^{*}$. The $G$-module map $\tau \Psi^{*} \tau: Y^{*} \otimes Y^{*} \rightarrow Y^{*} \otimes Y^{*}$ satisfies the braid equation because $\Psi^{*}$ does. One has the following isomorphism $\cong$ of vector spaces:

$$
\begin{aligned}
\mathcal{H}_{Y} & =\left(T\left(Y \oplus Y^{*}\right) \rtimes G\right) /<I_{\Psi}, I_{\tau \Psi^{*} \tau},\left\{f \otimes v-v \otimes f-\left\langle f, v^{(0)}\right\rangle v^{(1)}: f \in Y^{*}, v \in Y\right\}> \\
& \cong \mathcal{B}(Y, \Psi) \otimes \mathbb{C} G \otimes \mathcal{B}\left(Y^{*}, \tau \Psi^{*} \tau\right) .
\end{aligned}
$$

That is, the triangular decomposition of a braided Heisenberg double associated to a Yetter-Drinfeld module $Y$ is into the group algebra and two Nichols algebras.

8.2. Braided Heisenberg double as a semidirect product. Recall that $\delta: Y \rightarrow Y \otimes \mathbb{C} G$ denotes the coaction on $Y$ and $\Psi$ denotes the braiding on $Y$ defined by the Yetter-Drinfeld module structure. We write $\tau$ for the trivial braiding, $\tau(u \otimes v)=v \otimes u$ for $u, v \in Y$. As before, it is easy to see that $\tau \Psi \tau$ is also a braiding on $Y$. We start with the following lemma. 
Lemma 8.3. There exists an algebra automorphism $\widetilde{\delta}$ of $T(Y) \rtimes G$ defined by $\widetilde{\delta}(v)=\delta(v)$ and $\widetilde{\delta}(g)=g$ for $v \in Y, g \in G$. One has $\widetilde{\delta}\left(I_{\Psi} \otimes \mathbb{C} G\right)=I_{\tau \Psi \tau} \otimes \mathbb{C} G$, so that $\widetilde{\delta}$ induces an algebra isomorphism between $\mathcal{B}(Y, \Psi) \rtimes G$ and $\mathcal{B}(Y, \tau \Psi \tau) \rtimes G$.

Proof. The algebra $T(Y) \rtimes G$ is the quotient of $T(\mathbb{C} G \oplus Y)$ modulo the ideal generated by $g \otimes v-$ $(g \triangleright v) \otimes g$ and $g \otimes h-g h$, where $g, h \in G, v \in Y$ and $g h$ denotes the product of $g$ and $h$ in G. Consider the algebra endomorphism $\widetilde{\delta}$ of $T(\mathbb{C} G \oplus Y)$ defined on $\mathbb{C} G$ and on $Y$ as specified in the lemma. Then $\widetilde{\delta}$ preserves the relation $g \otimes h-g h$. Assuming $v \in Y_{x}$ where $x \in G, \widetilde{\delta}$ maps $g \otimes v-(g \triangleright v) \otimes g$ into $g \otimes v \otimes x-(g \triangleright v) \otimes g x g^{-1} \otimes g$, which is zero modulo the defining relations of $T(Y) \rtimes G$. Therefore, $\widetilde{\delta}$ is a well-defined algebra endomorphism of $T(Y) \rtimes G$. It is not difficult to see that the inverse $\widetilde{\delta}^{-1}$ of $\widetilde{\delta}$ is given by $\widetilde{\delta}^{-1}(g)=g$ and $\widetilde{\delta}^{-1}(v)=v \otimes x^{-1}$ for $v \in Y_{x}$. Hence $\widetilde{\delta}$ is an automorphism of $T(Y) \rtimes G$.

We will now compute the linear endomorphism $\widetilde{\delta}\left(\Psi \otimes \mathrm{id}_{\mathbb{C} G}\right) \widetilde{\delta}^{-1}$ of the vector space $Y \otimes Y \otimes \mathbb{C} G$. Let $u \in Y_{x}$ and $v \in Y_{y}$ with $x, y \in G$. Let $g \in G$. Then

$$
\begin{aligned}
& u \otimes v \otimes g \quad \stackrel{\widetilde{\delta}^{-1}}{\longrightarrow} u \otimes\left(x^{-1} \triangleright v\right) \otimes x^{-1} y^{-1} g \\
& \stackrel{\Psi \otimes \operatorname{id}_{\mathbb{C} G}}{\longrightarrow} x \triangleright\left(x^{-1} \triangleright v\right) \otimes u \otimes x^{-1} y^{-1} g=v \otimes u \otimes x^{-1} y^{-1} g \\
& \stackrel{\widetilde{\delta}}{\rightarrow} \quad v \otimes(y \triangleright u) \otimes g .
\end{aligned}
$$

This shows that $\widetilde{\delta}\left(\Psi \otimes \operatorname{id}_{\mathbb{C} G}\right) \widetilde{\delta}^{-1}=\tau \Psi \tau \otimes \operatorname{id}_{\mathbb{C} G}$. Now it easily follows that $\widetilde{\delta}\left([n] !_{\Psi} \otimes \operatorname{id}_{\mathbb{C} G}\right) \widetilde{\delta}^{-1}=$ $[n] !_{\tau \Psi \tau} \otimes \operatorname{id}_{\mathbb{C} G}$ as endomorphisms of $Y^{\otimes n} \otimes \mathbb{C} G$. Taking the kernels on both sides and summing over all $n \geq 2$ yields $\widetilde{\delta}\left(I_{\Psi} \otimes \mathbb{C} G\right)=I_{\tau \Psi \tau} \otimes \mathbb{C} G$.

Remark 8.4. The Nichols algebras $\mathcal{B}(Y, \Psi)$ and $\mathcal{B}(Y, \tau \Psi \tau)$ are, in general, not isomorphic. Rather, the identity map on $Y$ extends to an algebra isomorphism $\mathcal{B}(Y, \Psi) \stackrel{\sim}{\rightarrow} \mathcal{B}(Y, \tau \Psi \tau)^{\mathrm{op}}$. The isomorphism $\widetilde{\delta}$ between $\mathcal{B}(Y, \Psi) \rtimes G$ and $\mathcal{B}(Y, \tau \Psi \tau) \rtimes G$, constructed in Lemma 8.3. does not restrict to a map between $\mathcal{B}(Y, \Psi)$ and $\mathcal{B}(Y, \tau \Psi \tau)$.

Observe that the same result holds for $Y^{*}$ :

Corollary 8.5. The map $Y^{*} \rightarrow \mathbb{C} G \otimes Y^{*}$ defined by $f \in\left(Y^{*}\right)_{g} \mapsto g \otimes f$ extends to an algebra isomorphism $G \ltimes \mathcal{B}\left(Y^{*}\right) \stackrel{\sim}{\rightarrow} G \ltimes \mathcal{B}\left(Y^{*}, \tau \Psi^{*} \tau\right)$.

Definition 8.6. The braided Weyl algebra of a finite-dimensional Yetter-Drinfeld module $Y$ is

$$
\mathcal{A}_{Y}=\mathcal{B}(Y) \otimes \mathcal{B}\left(Y^{*}\right)
$$

as a vector space, where $\mathcal{B}(Y)$ and $\mathcal{B}\left(Y^{*}\right)$ embed as subalgebras, and the relations

$$
f v-(g \triangleright v) f=\langle f, v\rangle \cdot 1, \quad f \in\left(Y^{*}\right)_{g}, v \in Y
$$


hold, determining the multiplication in $\mathcal{A}_{Y}$ uniquely.

Remark 8.7. It follows from 5.7 that $\mathcal{B}(Y)$ and $\mathcal{B}\left(Y^{*}\right)$ are dually paired braided Hopf algebras in the category ${ }_{G} \mathcal{Y} \mathcal{D}^{G}$ : they are quotients of dually paired $T(Y)$ and $T\left(Y^{*}\right)$ modulo the respective kernels of the pairing. They have $Y$ and $Y^{*}$ as the respective spaces of primitive elements. It then follows from a general categorical construction of a braided Weyl algebra in [39] that the multiplication in $\mathcal{A}_{Y}$ is well-defined. In the case of a trivial $G$-grading on $Y, \mathcal{A}_{Y}$ is the ordinary Weyl algebra with underlying vector space $S(Y) \otimes S\left(Y^{*}\right)$ and defining relation $f v-v f=\langle f, v\rangle \cdot 1$ for $f \in Y^{*}, v \in Y$.

The following theorem is a version of [7, Proposition 1.23 and Example 1.25]: in [7, we dealt only with braided doubles with quadratic relations, whereas here we do not have that restriction.

Theorem 8.8. Let $Y$ be a finite-dimensional Yetter-Drinfeld module for a group $G$. Then there is an isomorphism

$$
\phi: \mathcal{A}_{Y} \rtimes G \stackrel{\sim}{\rightarrow} \mathcal{H}_{Y}
$$

of algebras, defined on generators $v \in Y, f \in Y^{*}$ and $g \in G$ of $\mathcal{A}_{Y} \rtimes G$ by $\phi(v)=v, \phi(f)=x \otimes f$ if $f \in\left(Y^{*}\right)_{x}$ where $x \in G$, and $\phi(g)=g$.

Proof. We already know from Corollary 8.5 that $\phi$, defined as above on $f \in Y^{*}$ and $g \in G$, extends to an algebra isomorphism between $G \ltimes \mathcal{B}\left(Y^{*}\right)$ and $G \ltimes \mathcal{B}\left(Y^{*}, \tau \Psi^{*} \tau\right) \subset \mathcal{H}_{Y}$. Trivially, $\left.\phi\right|_{Y}$ extends to an embedding of $\mathcal{B}(Y, \Psi)$ in $\mathcal{H}_{Y}$. To check that $\phi$ is well defined, it remains to show that $\phi$ maps the commutation relations between $Y^{*}, G$ and $Y$ in $\mathcal{A}_{Y}$ into relations of $\mathcal{H}_{Y}$. But this was done in [7. Proposition 1.23 and Example 1.25].

8.9. The braided Heisenberg double is a $G$-graded algebra. Our use of Theorem 8.8 is that it allows us to view the braided Heisenberg double $\mathcal{H}_{Y}$ as an algebra in the category ${ }_{G} \mathcal{Y D}^{G}$. Here, Examples 0.4 and 0.5 from the Introduction come together to produce a $G$-grading on $\mathcal{H}_{Y}$ :

Proposition 8.10. 1. The group $G$ acts on $\mathcal{H}_{Y}$ by conjugation: $g \triangleright x=g x g^{-1}$, and makes $\mathcal{H}_{Y}$ a G-algebra.

2. There is a G-grading on $\mathcal{H}_{Y} \cong \mathcal{B}(Y, \Psi) \otimes \mathbb{C} G \otimes \mathcal{B}\left(Y^{*}, \tau \Psi^{*} \tau\right)$ such that the $G$-degree of $g \in G$ is $g$, the $G$-degree of $v \in Y_{g}$ is $g$ and the $G$-degree of $Y^{*}$ is 1 .

3. The $G$-action and the $G$-grading make $\mathcal{H}_{Y}$ an algebra in the category ${ }_{G} \mathcal{Y D}^{G}$.

Proof. 1. is a standard fact about braided doubles, cf. Theorem 7.8 Note that, by the semidirect product relations, the adjoint action of $G$ extends the $G$-action on $\mathcal{B}(Y)$ and on $\mathcal{B}\left(Y^{*}, \tau \Psi^{*} \tau\right)$.

2. Recall the algebra isomorphism $\phi: \mathcal{A}_{Y} \rtimes G \rightarrow \mathcal{H}_{Y}$ from Theorem 8.8, By the general construction in [39], the braided Weyl algebra $\mathcal{A}_{Y} \cong \mathcal{B}(Y) \otimes \mathcal{B}\left(Y^{*}\right)$ is an algebra in the category ${ }_{G} \mathcal{Y} \mathcal{D}^{G}$, so 
is $G$-graded by extending the $G$-grading from $Y$ and $Y^{*}$. This grading and the natural $G$-grading on $\mathbb{C} G$ extend to a $G$-grading on $\mathcal{A}_{Y} \rtimes G$. This, in turn, induces a $G$-grading on $\mathcal{H}_{Y}$ via the isomorphism $\phi$. The result is exactly as described in the Proposition. In particular, if $f \in\left(Y^{*}\right)_{x}$, then $\phi(f)=x f$ has degree $x$ in $\mathcal{H}_{Y}$ which means that $f$ must have trivial $G$-degree in $\mathcal{H}_{Y}$.

3. The Yetter-Drinfeld condition on $\mathcal{H}_{Y}$ easily follows from the said condition on $\mathcal{B}(Y)$ and on $(\mathbb{C} G)_{\mathrm{ad}}$.

The $G$-grading on a braided Heisenberg double $\mathcal{H}_{Y}$ allows us to extend $\mathcal{H}_{Y}$ by cocycles. We do this in the next theorem.

Theorem 8.11 (Cocycle extension of a braided Heisenberg double). Let $G$ be a finite group, $Y$ be a finite-dimensional Yetter-Drinfeld module for $\mathbb{C} G, R$ be a commutative $\mathbb{C}$-algebra and $\mu \in$ $Z^{2}\left(G, R^{\times}\right)$. The extension of $\mathcal{H}_{Y}$ by the cocycle $\mu$ is an algebra in the category ${ }_{R, G} \mathcal{Y D}^{G}$ with the following triangular decomposition:

$$
\left(\widetilde{\mathcal{H}_{Y}}\right)_{\mu} \cong \mathcal{B}\left(\widetilde{Y}_{\mu}\right) \underset{R}{\otimes} R G_{\mu} \underset{R}{\otimes} \mathcal{B}\left(\widetilde{Y}^{*}, \tau \Psi^{*} \tau\right)
$$

Here $\tilde{Y}_{\mu}$ is as defined in Corollary 6.17 and $\tilde{Y}^{*}=R \otimes_{\mathbb{C}} Y^{*}$ is the trivial extension of $Y^{*}$. The product $\star$ on $\left.\widetilde{\left(\mathcal{H}_{Y}\right.}\right)_{\mu}$ is completely described by the following. The R-submodules $A_{-1}=\mathcal{B}\left(\widetilde{Y}_{\mu}\right)$, $A_{0}=R G_{\mu}, A_{1}=\mathcal{B}\left(\widetilde{Y}^{*}, \tau \Psi^{*} \tau\right)$ are subalgebras over $R$, as are $A_{-1} A_{0}$ and $A_{0} A_{1}$. The relations

$$
\begin{aligned}
& g \star v=\left(g \triangleright_{\chi} v\right) \star g, \quad g \star f=(g \triangleright f) \star g, \\
& f \star v-v \star f=\left\langle f, v^{(0)}\right\rangle v^{(1)} \quad \text { for } g \in G, v \in Y, f \in Y^{*}
\end{aligned}
$$

hold where $\chi=\chi_{\mu}$ and $\triangleright$ is the original action of $G$ on $Y$ and $Y^{*}$.

Proof. The first part (tensor decomposition) follows from Proposition 8.10 and Corollary 6.17 Let us verify the cross-commutation relations between $Y, G$ and $Y^{*}$ in $\left.\widetilde{\left(\mathcal{H}_{Y}\right.}\right)_{\mu}$. Assume that $v \in Y_{h}$ where $h \in G$. By definition of $\star$ one has $g \star v=\mu(g, h) g v=\mu(g, h)(g \triangleright v) g$ which can be written as $\mu(g, h) \mu\left(g h g^{-1}, g\right)^{-1}(g \triangleright v) \star g=\left(g \triangleright_{\chi} v\right) \star g$. Furthermore, since $\mu$ is a normalised cocycle and $f$, $g \triangleright f \in Y^{*}$ have $G$-degree 1 , one has $g \star f=g f=(g \triangleright f) g=(g \triangleright f) \star g$, and $f \star v-v \star f=f v-v f$ is as given in 8.1 .

8.12. The case $R=\mathbb{C} \Gamma$. Consider a special case of the cocycle extension of $\mathcal{H}_{Y}$ where $\Gamma$ an abelian group, $R=\mathbb{C} \Gamma$ and $\mu \in Z^{2}(G, \Gamma)$. Note that

$$
R G_{\mu} \cong \mathbb{C} \widetilde{G}_{\mu}
$$

where $\mathbb{C} \widetilde{G}_{\mu}$ is the group algebra of the central extension $\widetilde{G}_{\mu}$ of $G$ by $\Gamma$. Explicitly, recall that $\widetilde{G}_{\mu}$, as a set, is the cartesian product $\Gamma \times G=\{(z, g) \mid z \in \Gamma, g \in G\}$, whereby the above isomorphism is

$$
z g \mapsto(z, g), \quad z \in \Gamma, \quad g \in G .
$$


Recall the monoidal category $\Gamma$ - Vect introduced in 7.13. Theorem 8.11 implies the following

Corollary 8.13. The cocycle extension $\left(\widetilde{\mathcal{H}_{Y}}\right)_{\mu}$ of $\mathcal{H}_{Y}$ is a braided double in the category $\Gamma$ - Vect.

As a vector space over $\mathbb{C}$, the algebra $\left(\widetilde{\mathcal{H}_{Y}}\right)_{\mu}$ has the tensor decomposition

$$
\mathcal{B}(Y) \otimes \mathbb{C} \Gamma \otimes \mathbb{C} G \otimes \mathcal{B}\left(Y^{*}, \tau \Psi^{*} \tau\right)
$$

However, the subspace $\mathcal{B}(Y)$ is not a subalgebra of $\left(\widetilde{\mathcal{H}_{Y}}\right)_{\mu}$, because the relations between elements of $Y$ in $\left(\widetilde{\mathcal{H}_{Y}}\right)_{\mu}$ involve elements of the group $\Gamma$. Thus, the extension $\left(\widetilde{\mathcal{H}_{Y}}\right)_{\mu}$ with $\mu \in Z^{2}(G, \Gamma)$ has triangular decomposition over $\mathbb{C} \Gamma$ but not over $\mathbb{C}$.

As one might expect, if the cocycle $\mu$ is scalar-valued, the twist of $\mathcal{H}_{Y}$ by $\mu$ will have triangular decomposition over $\mathbb{C}$. Denote by $\left(Y, \triangleright_{\chi}\right)$ the Yetter-Drinfeld module which is the same $G$-graded space as $Y$ but where $G$ acts by $\triangleright_{\chi}$. The above arguments can be easily seen to imply

Corollary 8.14. If $\mu \in Z^{2}\left(G, \mathbb{C}^{\times}\right)$, the twist $\left(\mathcal{H}_{Y}\right)_{\mu}$ has triangular decomposition

$$
\mathcal{B}\left(Y, \triangleright_{\chi}\right) \otimes \mathbb{C} G_{\mu} \otimes \mathcal{B}\left(Y^{*}, \tau \Psi^{*} \tau\right)
$$

with cross-commutation relations $g \star v=\left(g \triangleright_{\chi} v\right) \star g, g \star f=(g \triangleright f) \star g, f \star v-v \star f=\left\langle f, v^{(0)}\right\rangle v^{(1)}$ for $g \in G, v \in Y, f \in Y^{*}$, where $\chi=\chi_{\mu}$.

Note that the cocycle twist $\left(\mathcal{H}_{Y}\right)_{\mu}$ is an algebra with triangular decomposition but not a braided double: the twisted group algebra $\mathbb{C} G_{\mu}$ is not, in general, a Hopf algebra.

\section{EXTENSIONS AND TWISTS OF OTHER BRAIDED DOUBLES}

We now turn to braided doubles over $G$ which are not Heisenberg. They will not in general be $G$-graded. However, a construction which we describe in this section realises some braided doubles as subdoubles of braided Heisenberg doubles. We then define extensions of braided doubles, for simplicity restricting ourselves to cocycles $\mu \in Z^{2}(G, \Gamma)$ where $\Gamma$ is an abelian group.

9.1. Subdoubles of braided Heisenberg doubles. Let $Y$ be a finite-dimensional Yetter-Drinfeld module for $G$, and let $V^{-} \subset Y, V^{+} \subset Y^{*}$ be $G$-submodules, not necessarily $G$-graded. Denote by $A_{V^{-}, V^{+}}$the subalgebra of $\mathcal{H}_{Y}$ generated by $V^{-}, G$ and $V^{+}$. Then $A_{V^{-}, V^{+}}$has the structure of a braided double on $V^{-}$and $V^{+}$, with triangular decomposition

$$
A_{V^{-}, V^{+}} \cong U^{-} \otimes \mathbb{C} G \otimes U^{+} \quad \subset \quad \mathcal{H}_{Y} \cong \mathcal{B}(Y, \Psi) \otimes \mathbb{C} G \otimes \mathcal{B}\left(Y^{*}, \tau \Psi^{*} \tau\right)
$$

Here $\Psi$ is the braiding on the Yetter-Drinfeld module $Y$ and $\mathcal{B}(Y, \Psi)$ is the Nichols algebra of $Y$. The subalgebra $U^{-}=T\left(V^{-}\right) / T\left(V^{-}\right) \cap I_{\Psi}$ of $\mathcal{B}(Y, \Psi)$ is generated in degree one by $V^{-}$; similarly for 
$U^{+}$. Note that both $U^{-}$and $U^{+}$are graded but not necessarily $G$-graded algebras. The commutator between $f \in V^{+}$and $v \in V^{-}$in $A_{V^{-}, V^{+}}$is defined as their commutator in $\mathcal{H}_{Y}$ :

$$
\beta_{V^{+}, V^{-}}(f \otimes v)=f v-v f \in \mathbb{C} G \subset \mathcal{H}_{Y},
$$

so that

$$
A_{V^{-}, V^{+}}=A_{\beta_{V^{+}, V^{-}}}\left(T\left(V^{-}\right) \cap I_{\Psi}, T\left(V^{+}\right) \cap I_{\tau \Psi^{*} \tau}\right) .
$$

9.2. A counterexample to minimality of $A_{V^{-}, V^{+}}$. In general, the braided double $A_{V^{-}, V^{+}}=$ $A_{\beta_{V^{+}, V^{-}}}\left(T\left(V^{-}\right) \cap I_{\Psi}, T\left(V^{+}\right) \cap I_{\tau \Psi^{*} \tau}\right)$ is not minimal, although it is embedded in a minimal double $\mathcal{H}_{Y}$. An extreme example is where $V^{-}$and $V^{+}$are Yetter-Drinfeld submodules of $Y, Y^{*}$ orthogonal with respect to the evaluation pairing between $Y^{*}$ and $Y$. Then $V^{-}$and $V^{+}$commute in $\mathcal{H}_{Y}$, so that $\beta_{V^{+}, V^{-}}=0$. The minimal double associated to $\beta_{V^{+}, V^{-}}$is $\bar{A}_{0}$ with triangular decomposition $\mathbb{C} \otimes \mathbb{C} G \otimes \mathbb{C} \cong \mathbb{C} G$. This is not the same as $A_{V^{-}, V^{+}}$.

9.3. The embedding theorem. The following theorem shows that for any $\beta$, there exists a braided double $A_{\beta}\left(I^{-}, I^{+}\right)$with some relations - not necessarily a minimal double — which embeds in a braided Heisenberg double. We will not give a proof of this theorem because it follows from [6, Theorem 6.9], subject to minor modifications explained above in Remark 7.9.

Recall that a morphism $f$ between two braided doubles over $G$ is determined by the restriction of $f$ to degree one generators, see 7.10 .

Theorem 9.4. Let $G$ be a finite group, $V^{ \pm}$be $G$-modules, $\operatorname{dim} V^{ \pm}<\infty$. Let $\beta: V^{+} \otimes V^{-} \rightarrow \mathbb{C} G$ be a $G$-equivariant map. Let $A_{\beta}(0,0)$ denote the free braided double with triangular decomposition $T\left(V^{-}\right) \otimes \mathbb{C} G \otimes T\left(V^{+}\right)$. There exists a finite-dimensional Yetter-Drinfeld module $Y$ and a morphism

$$
f: A_{\beta}(0,0) \rightarrow \mathcal{H}_{Y}
$$

of braided doubles. If $I^{ \pm}=\left.\operatorname{ker} f\right|_{T\left(V^{ \pm}\right)} \subset T\left(V^{ \pm}\right)$, the morphism $f$ induces an embedding

$$
A_{\beta}\left(I^{-}, I^{+}\right) \hookrightarrow \mathcal{H}_{Y}
$$

of braided doubles over $G$.

9.5. Extensions and twists of braided doubles. Let $V^{-}, V^{+}$be finite-dimensional $G$-modules, $\beta: V^{+} \otimes V^{-} \rightarrow \mathbb{C} G$ be a $G$-equivariant map, and $A=A_{\beta}\left(I^{-}, I^{+}\right)$be a braided double where $I^{ \pm}$is a graded two-sided $G$-invariant ideal of $T\left(V^{ \pm}\right)$. In general, $A$ is not a $G$-graded algebra. However, motivated by the Embedding Theorem 9.4, we assume that there is a finite-dimensional Yetter-Drinfeld module $Y$ for $G$ and a morphism

$$
f: A \rightarrow \mathcal{H}_{Y}
$$


of braided doubles over $G$.

Let $\Gamma$ be an abelian group and $\mu \in Z^{2}(G, \Gamma)$. We would like to extend the braided double $A$ by the cocycle $\mu$. To simplify notation, assume $\Gamma=C_{k}=\left\langle z \mid z^{k}=1\right\rangle$ and denote the specialisation map by $\left.\cdot\right|_{z=1}$.

Definition 9.6. In the above setup, an extension of $A$ by $\mu$ covering the morphism $f$ is a commutative diagram

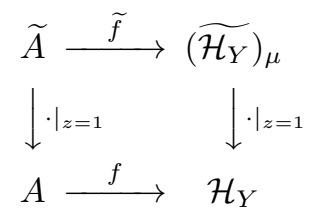

where $\widetilde{A}$ is a braided double in $\Gamma$ - Vect and $\widetilde{f}$ is a morphism of braided doubles in $\Gamma$ - Vect. We will also say that $\widetilde{A}$ is the extension of $A$ if the rest of the diagram is implied.

We make some remarks about this definition. Observe that, while cocycle extensions of braided Heisenberg doubles are canonical, extensions of other braided doubles are not, and in general they depend on $f$. An extension of $A$ by a given cocycle $\mu$ covering a given morphism $f$ is not guaranteed to exist; we do not know if it is unique. Note that, while $f(A)$ is a subdouble of $\mathcal{H}_{Y}$ and is itself a braided double, $\widetilde{f}(\widetilde{A})$ may not be a braided double in $\Gamma$-Vect because it may fail to be a free $\Gamma$-module. So an extension of $A$ does not necessarily give an extension of $f(A)$. An example of this situation is given in the next section.

Motivated by Corollary 3.9 we now define a cocycle twist of a braided double as follows.

Definition 9.7 (Cocycle twist of a braided double). In the above setting, a cocycle twist of a braided double $A$ is a specialisation at $z=q$ of some extension of $A$ by a cocycle $\mu \in Z^{2}\left(G, C_{k}\right)$. Here $q \in \mathbb{C}^{\times}$is a $k$ th root of 1 .

\section{Covering Cherednik algebras and spin Cherednik algebras}

In this last section of the paper, we apply the cocycle extension and cocycle twist techniques to rational Cherednik algebras $H_{0, c}\left(S_{n}\right)$ to produce new algebras with triangular decomposition.

10.1. The rational Cherednik algebra. Let $n$ be fixed and let $t, c \in \mathbb{C}$. The rational Cherednik algebra of the group $S_{n}$, denoted $H_{t, c}$, was introduced by Etingof and Ginzburg in [24, §4] as a degenerate version of the double affine Hecke algebra of Cherednik. The algebra $H_{t, c}$ has generators $x_{1}, \ldots, x_{n}$, the group $S_{n}$, and $y_{1}, \ldots, y_{n}$ and relations

$$
\begin{aligned}
& x_{i} x_{j}=x_{j} x_{i}, \quad y_{i} y_{j}=y_{j} y_{i}, \quad \sigma x_{i}=x_{\sigma(i)} \sigma, \quad \sigma y_{i}=y_{\sigma(i)} \sigma, \\
& y_{i} x_{j}-x_{j} y_{i}=c \cdot(i j), \quad i \neq j ;
\end{aligned}
$$


Here $1 \leq i, j \leq n$ and $\sigma \in S_{n}$. By the Poincaré-Birkhoff-Witt type theorem for rational Cherednik algebras proved in 24], $H_{t, c}$ is the braided double $A_{\beta}\left(\wedge^{2} V^{-}, \wedge^{2} V^{+}\right)$, where $V^{-}$is the $\mathbb{C}$-linear span of $x_{1}, \ldots, x_{n}$ and $V^{+}$is the span of $y_{1}, \ldots, y_{n}$. The map $\beta: V^{+} \otimes V^{-} \rightarrow \mathbb{C} S_{n}$ can be read off the commutator relations between the $y_{i}$ and $x_{j}$ above.

For the rest of the paper we assume $t=0$. In this case the rational Cherednik algebra has an interesting cocycle extension which is an algebra over $\mathbb{C C}_{2}$.

\subsection{The reduced Cherednik algebra and its embedding in a braided Heisenberg double.}

The braided double $H_{0, c}$ is not minimal and admits a finite-dimensional quotient, the minimal double. It was shown in [6, Proposition 7.13] that if $c \neq 0$, this minimal double is the reduced Cherednik algebra introduced by Gordon [29]:

$$
\bar{H}_{0, c} \cong P_{n} \otimes \mathbb{C} S_{n} \otimes P_{n}^{\vee}
$$

where $P_{n}=\mathbb{C}\left[x_{1}, \ldots, x_{n}\right] /<f_{1}, \ldots, f_{n}>$ is the coinvariant algebra of $S_{n}$, the $f_{i}$ being the elementary symmetric functions, $\operatorname{deg} f_{i}=i$. (Here $P_{n}^{\vee}$ denotes a copy of $P_{n}$ generated by $y_{1}, \ldots, y_{n}$.) One has $\operatorname{dim} P_{n}=\operatorname{dim} P_{n}^{\vee}=n !$ and $\operatorname{dim} \bar{H}_{0, c}=(n !)^{3}$. We note the morphism

$$
f: H_{0, c} \rightarrow \bar{H}_{0, c}
$$

of braided doubles.

Let $X_{n}$ denote the set of transposition in $S_{n}$, and let

$$
Y_{n}:=\left(X_{n}, q_{1}\right)
$$

be the Yetter-Drinfeld module for $\mathbb{C} S_{n}$ described in 6.18. The underlying vector space of $Y_{n}$ is $\mathbb{C} X_{n}=\mathbb{C}-\operatorname{span}\left\{e_{\tau}: \tau \in X_{n}\right\}$. To distinguish $Y_{n}$ from its dual space, we assume that $Y_{n}^{*}$ is spanned by $\left\{e_{\tau}^{*}: \tau \in X_{n}\right\}$, a basis dual to $\left\{e_{\tau}\right\}$. Note that, by definition of the braided Heisenberg double in 8.1 ,

$$
e_{\tau}^{*} e_{v}-e_{v} e_{\tau}^{*}=\delta_{\tau v} \tau \in \mathcal{H}_{Y_{n}}, \quad \tau, v \in X_{n}
$$

We recall the embedding of $\bar{H}_{0, c}$ in the braided Heisenberg double $\mathcal{H}_{Y_{n}}$, constructed in [6] for the more general situation of a complex reflection group. Here we quote the result from [6] in the case of $S_{n}$, noting the appearance of the Fomin-Kirillov "Dunkl elements" $\theta_{j} \in Y_{n}$ as in 6.18, We denote by $\theta_{j}^{*}$ a copy of $\theta_{j}$ in the space $Y_{n}^{*}$ : 
Proposition 10.3 (see [6]). Let $c \neq 0$. There is an embedding of $\bar{H}_{0, c}$ as a subdouble in the braided Heisenberg double $\mathcal{H}_{Y_{n}}$ given by

$$
\begin{aligned}
& x_{j} \mapsto \theta_{j}=-\sum_{1 \leq i<j} e_{(i j)}+\sum_{j<i \leq n} e_{(j i)} \in Y_{n}, \\
& y_{j} \mapsto-c \theta_{j}^{*}=-c\left(-\sum_{1 \leq i<j} e_{(i j)}^{*}+\sum_{j<i \leq n} e_{(j i)}^{*}\right) \in Y_{n}^{*},
\end{aligned}
$$

and $\sigma \mapsto \sigma$ for $\sigma \in S_{n}$. Therefore, the morphism $f: H_{0, c} \rightarrow \bar{H}_{0, c}$ can be viewed as the morphism

$$
f: H_{0, c} \rightarrow \mathcal{H}_{Y_{n}}, \quad f\left(x_{j}\right)=\theta_{j}, \quad f\left(y_{j}\right)=-c \theta_{j}^{*}
$$

of braided doubles.

10.4. The covering Cherednik algebra $\widetilde{H}_{0, c}$. We will now construct an extension of $H_{0, c}$ by a cocycle $\mu \in Z^{2}\left(S_{n}, C_{2}\right)$. We are going to use the cocycle we studied above in 2.8 , namely

$$
\mu=[1, z]
$$

This is a non-trivial cocycle if $n \geq 4$, which we will assume from now on. Our extension will be covering the above braided double morphism $f$, which means that we are looking for a commutative diagram

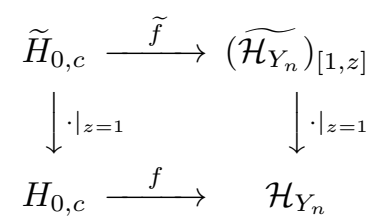

Denote $\mathbb{C} C_{2}$ by $R$ and recall the triangular decomposition

$$
\left(\widetilde{\mathcal{H}_{Y_{n}}}\right)_{\mu} \cong \mathcal{B}\left(R X_{n}, q_{z}\right) \underset{R}{\otimes} \mathbb{C} T_{n} \underset{R}{\otimes} \mathcal{B}\left(\widetilde{Y}_{n}^{*}\right)
$$

in $C_{2}$-Vect, where $T_{n}=\left(\widetilde{S_{n}}\right)_{[1, z]}$ is the Schur covering group of $S_{n}$, see Theorem 2.6. The module $\left(\widetilde{Y_{n}}\right)_{[1, z]}=\left(R X_{n}, q_{z}\right) \in \operatorname{Ob}\left(R, S_{n} \mathcal{Y} \mathcal{D}^{S_{n}}\right)$ was defined in $\left[6.19\right.$, and $\widetilde{Y}_{n}^{*}=R \otimes_{\mathbb{C}} Y_{n}^{*}$ is a trivial extension of the Yetter-Drinfeld module $Y_{n}^{*}$. Explicitly,

$$
\mathcal{B}\left(\widetilde{Y}_{n}^{*}\right) \cong R \underset{\mathbb{C}}{\otimes} \mathcal{B}\left(Y_{n}\right)
$$

where the isomorphism just relabels the generators $e_{\tau}, \tau \in X_{n}$ on the right as $e_{\tau}^{*}$ on the left. This is because the Yetter-Drinfeld module $Y_{n}$ is self-dual, cf. [5].

The algebra $\widetilde{H}_{0, c}$ over $R$ must be a $C_{2}$-flat deformation of $H_{0, c}$. It will be generated by $\widetilde{x}_{1}, \ldots, \widetilde{x}_{n}$, $T_{n}$ and $\widetilde{y}_{1}, \ldots, \widetilde{y}_{n}$, where $\widetilde{x}_{i}$ is some choice of a preimage of $x_{i}$ in $\widetilde{H}_{0, c}$, i.e., $\left.\widetilde{x}_{i}\right|_{z=1}=x_{i}$. Because of the flatness of deformation requirement, we should lift the commutation relations between the $x_{i}$ in such a way that the $\widetilde{x}_{i}$ generate the subalgebra of $\widetilde{H}_{0, c}$ isomorphic, as an $R$-module, to $R \otimes_{\mathbb{C}} \mathbb{C}\left[x_{1}, \ldots, x_{n}\right]$. 
Therefore, it makes sense to study the commutation relations between $\widetilde{f}\left(\widetilde{x}_{1}\right), \ldots, \widetilde{f}\left(\widetilde{x}_{n}\right)$ in the algebra $\mathcal{B}\left(R X_{n}, q_{z}\right)$. Note that $\widetilde{f}\left(\widetilde{x}_{j}\right)$ is the lift of $\theta_{j} \in Y_{n}$ to $\left(\widetilde{Y_{n}}\right)_{\mu}$. (Also, $\widetilde{f}\left(\widetilde{y}_{j}\right)$ is a lift of $\theta_{j}^{*}$ to $\widetilde{Y}_{n}^{*}$, but because there is no twisting applied to $\mathcal{B}\left(Y_{n}^{*}\right)$ to get $\mathcal{B}\left(\widetilde{Y}_{n}^{*}\right)$, any lifts of the $\theta_{j}^{*}$ will commute in $\left.\mathcal{B}\left(\tilde{Y}_{n}^{*}\right).\right)$

In choosing the correct lift of the $\theta_{j}$, we are guided by Majid's formula for the flat connections $\alpha_{j}$, see 6.18 The result is the following proposition, which plays a crucial role in constructing the algebra $\widetilde{H}_{0, c}$.

Proposition 10.5. The elements

$$
\widetilde{\theta}_{j}=-\sum_{1 \leq i<j} e_{(i j)}+z \sum_{j<i \leq n} e_{(j i)} \quad \in R X_{n}, \quad j=1, \ldots, n,
$$

$z$-commute in the algebra $\mathcal{B}\left(R X_{n}, q_{z}\right)$, that is, $\widetilde{\theta}_{i} \widetilde{\theta}_{j}=z \widetilde{\theta}_{j} \widetilde{\theta}_{i}$ if $i \neq j$.

Proof. Consider the quadratic element $u_{i j}=\widetilde{\theta}_{i} \widetilde{\theta}_{j}-z \widetilde{\theta}_{j} \widetilde{\theta}_{i} \in \mathcal{B}\left(R X_{n}, q_{z}\right)$. If we specialise $z$ to 1 , $u_{i j}$ becomes the commutator of the truncated Dunkl elements $\theta_{i}, \theta_{j} \in \mathcal{B}\left(Y_{n}\right)$ of Fomin-Kirillov. So by a result of [26, $\left.u_{i j}\right|_{z=1}=0$. Now consider $\left.u_{i j}\right|_{z=-1}$. This is the anticommutator of the flat connections $\alpha_{i}$ and $\alpha_{j}$ in Majid's algebra $\Lambda_{n}$. So by a result of [43], $\left.u_{i j}\right|_{z=-1}=0$. Recall the direct sum decomposition $\mathcal{B}\left(R X_{n}, q_{z}\right) \cong \frac{1+z}{2} \mathcal{B}\left(X_{n}, q_{1}\right) \oplus \frac{1-z}{2} \mathcal{B}\left(X_{n}, q_{-1}\right)$ from Theorem 6.20. That both specialisations of $u_{i j}$ are zero means that the projections of $u_{i j}$ onto both summands of this direct sum are zero. But this means $u_{i j}=0$ in $\mathcal{B}\left(R X_{n}, q_{z}\right)$, as required. Alternatively, the Proposition may be deduced from the quadratic relations in $\mathcal{B}\left(R X_{n}, q_{z}\right)$ given at the end of Section 6

We also denote

$$
\widetilde{\theta}_{j}^{*}=-\sum_{1 \leq i<j} e_{(i j)}^{*}+\sum_{j<i \leq n} e_{(j i)}^{*} \in R X_{n},
$$

i.e., $\widetilde{\theta}_{j}^{*}$ is the trivial lift of $\theta_{j}^{*}$ from $Y_{n}^{*}$ to $\widetilde{Y}_{n}^{*}=R \otimes_{\mathbb{C}} Y_{n}^{*}$. Note that the $\widetilde{\theta}_{j}^{*}$ commute in $\mathcal{B}\left(\widetilde{Y}_{n}^{*}\right)$.

Let us now see what are the cross-commutator relations between the $\widetilde{\theta}_{j}$, the elements of the group $T_{n}$ and the $\widetilde{\theta}_{i}^{*}$ in the algebra $\left(\widetilde{\mathcal{H}_{Y_{n}}}\right)_{[1, z]}$. Recall from 2.5 that elements of $T_{n}$ can be written as $(1, \sigma)$ of $(z, \sigma)$ with $\sigma \in S_{n}$. In particular, $t_{i}=(1,(i i+1))$. The group product $\star_{[1, z]}$ on $T_{n}$ is twisted by the cocycle $[1, z]$. Let

$$
t_{i j}= \begin{cases}(1,(i j)), & \text { if } i<j, \\ (z,(i j)), & \text { if } i>j,\end{cases}
$$

so that $t_{i j}$ and $t_{j i}$ are the two lifts of the transposition $(i j)$ to the Schur covering group $T_{n}$, and $t_{i}=t_{i, i+1}$. Note that, by Theorem 8.11 and the explanation of the case $R=\mathbb{C} \Gamma$ in 8.12 ,

$$
\text { if } i<j,(i j) \neq(k l), \quad e_{(i j)}^{*} e_{(i j)}-e_{(i j)} e_{(i j)}^{*}=t_{i j} ; \quad e_{(i j)}^{*} e_{(k l)}-e_{(k l)} e_{(i j)}^{*}=0
$$

in the algebra $\left(\widetilde{\mathcal{H}_{Y_{n}}}\right)_{[1, z]}$. 
Lemma 10.6. The following relations hold in the algebra $\left(\widetilde{\mathcal{H}_{Y_{n}}}\right)_{[1, z]}$ :
(a) $t_{i} \widetilde{\theta}_{j}=z \widetilde{\theta}_{j} t_{i}, \quad t_{i} \widetilde{\theta}_{j}^{*}=\widetilde{\theta}_{j}^{*} t_{i}, \quad j \neq i, i+1 ;$
(b) $t_{i} \widetilde{\theta}_{i}=\widetilde{\theta}_{i+1} t_{i}, \quad t_{i} \widetilde{\theta}_{i}^{*}=\widetilde{\theta}_{i+1}^{*} t_{i}$;
(c) $\widetilde{\theta}_{i}^{*} \widetilde{\theta}_{j}-\widetilde{\theta}_{j} \widetilde{\theta}_{i}^{*}=-t_{i j}, \quad j \neq i$;
(d) $\widetilde{\theta}_{i}^{*} \widetilde{\theta}_{i}-\widetilde{\theta}_{i} \widetilde{\theta}_{i}^{*}=\sum_{k \neq i} t_{k i}$.

Proof. If $\sigma \in S_{n}$ and $\widetilde{\sigma}$ is any one of the two elements of $T_{n}$ satisfying $\left.\widetilde{\sigma}\right|_{z=1}=\sigma$, then $\widetilde{\sigma} e_{\tau} \widetilde{\sigma}^{-1}=$ $q_{z}(\sigma, \tau) e_{\sigma \tau \sigma^{-1}}$ in $\left(\widetilde{\mathcal{H}_{Y_{n}}}\right)_{[1, z]}$. The function $q_{z} \in Z^{1}\left(S_{n}, F u n\left(X_{n}, R^{\times}\right)\right)$was defined in 6.19, In particular, if $\sigma=(i i+1)$, this gives $t_{i} e_{\tau}=z e_{\tau} t_{i}$ whenever $\tau \neq(i i+1)$. Observe that $t_{i} e_{\tau}^{*}=e_{\tau}^{*} t_{i}$ whenever $\tau \neq(i i+1)$ because the module $\tilde{Y}_{n}^{*}$ is not twisted. So (a) follows by direct verification. Also, $t_{i} e_{(i i+1)}=-e_{(i i+1)} t_{i}$ and $t_{i} e_{(i i+1)}^{*}=-e_{(i i+1)}^{*} t_{i}$ and which implies (b). For (c), use the commutation relations between $e_{(i j)}$ and $e_{(k l)}$ given before the Lemma, and observe that the commutator $\left[\widetilde{\theta}_{i}^{*}, \widetilde{\theta}_{j}\right]$ is equal to $\left[e_{(i j)}^{*},-e_{(i j)}\right]$ if $i<j$ and to $\left[-e_{(i j)}^{*}, z e_{(i j)}\right]$ if $i>j$. In both cases the result is $-t_{i j}$. Finally, observe that $\left[\widetilde{\theta}_{i}^{*}, \widetilde{\theta}_{i}\right]=\sum_{k<i}\left[-e_{(k i)}^{*},-e_{(k i)}\right]+\sum_{k>i}\left[e_{(i k)}^{*}, z e_{(i k)}\right]$, so (d) easily follows.

We are now ready to construct the covering Cherednik algebra.

Definition 10.7. $\widetilde{H}_{0, c}$ is the $\mathbb{C}$-algebra with generators

$$
z, \quad \widetilde{x}_{1}, \ldots, \widetilde{x}_{n}, \quad t_{1}, \ldots, t_{n-1}, \quad \widetilde{y}_{1}, \ldots, \widetilde{y}_{n}
$$

and relations

(i) $z$ is central, $z^{2}=1$;

(ii) Schur's $T_{n}$ relations between $z, t_{1}, \ldots, t_{n}$ from Theorem 2.6.

(iii) $\widetilde{x}_{i} \widetilde{x}_{j}=z \widetilde{x}_{j} \widetilde{x}_{i}, \quad i \neq j$;

(iv) $\widetilde{y}_{i} \widetilde{y}_{j}=\widetilde{y}_{j} \widetilde{y}_{i}, \quad i \neq j$;

(v) $t_{i} \widetilde{x}_{j}=z \widetilde{x}_{j} t_{i}, \quad t_{i} \widetilde{y}_{j}=\widetilde{y}_{j} t_{i}, \quad j \neq i, i+1$

(vi) $t_{i} \widetilde{x}_{i}=\widetilde{x}_{i+1} t_{i}, \quad t_{i} \widetilde{y}_{i}=\widetilde{y}_{i+1} t_{i}$;

(vii) $\widetilde{y}_{i} \widetilde{x}_{j}-\widetilde{x}_{j} \widetilde{y}_{i}=c t_{i j}, \quad j \neq i$;

(viii) $\widetilde{y}_{i} \widetilde{x}_{i}-\widetilde{x}_{i} \widetilde{y}_{i}=-c \sum_{k \neq i} t_{k i}$.

Denote by $(\mathbb{C} \Gamma)\left[\widetilde{x}_{1}, \ldots, \widetilde{x}_{n}\right]_{z}$ an algebra over $\mathbb{C} \Gamma=\mathbb{C} C_{2}$ generated by $\widetilde{x}_{1}, \ldots, \widetilde{x}_{n}$ subject to the relations (iii) above. This is a $z$-commutative analogue of the polynomial algebra in the category $\Gamma$ - Vect. As $\mathbb{C} \Gamma$-modules (not as algebras), $(\mathbb{C} \Gamma)\left[\widetilde{x}_{1}, \ldots, \widetilde{x}_{n}\right]_{z} \cong \mathbb{C} \Gamma \otimes_{\mathbb{C}} \mathbb{C}\left[\widetilde{x}_{1}, \ldots, \widetilde{x}_{n}\right]$. On the other hand, the algebra $(\mathbb{C} \Gamma)\left[\widetilde{y}_{1}, \ldots, \widetilde{y}_{n}\right]$ (without the $z$ subscript) is a commutative polynomial algebra. 
Theorem 10.8. The algebra $\widetilde{H}_{0, c}$ is a braided double in the category $\Gamma$-Vect, where $\Gamma=C_{2}$, with triangular decomposition

$$
\widetilde{H}_{0, c} \cong(\mathbb{C} \Gamma)\left[\widetilde{x}_{1}, \ldots, \widetilde{x}_{n}\right]_{z} \underset{\mathbb{C} \Gamma}{\otimes} \mathbb{C} T_{n} \underset{\mathbb{C} \Gamma}{\otimes}(\mathbb{C} \Gamma)\left[\widetilde{y}_{1}, \ldots, \widetilde{y}_{n}\right]
$$

That is, the elements

$$
\widetilde{x}_{1}^{k_{1}} \ldots \widetilde{x}_{n}^{k_{n}}(1, \sigma) \widetilde{y}_{1}^{l_{1}} \ldots \widetilde{y}_{n}^{l_{n}}, \quad k_{i}, l_{i} \geq 0, \quad \sigma \in S_{n}
$$

are a basis of $\widetilde{H}_{0, c}$ as a free $\mathbb{C} \Gamma$-module. There is a morphism

$$
\widetilde{f}: \widetilde{H}_{0, c} \rightarrow\left(\widetilde{\mathcal{H}_{Y_{n}}}\right)_{[1, z]}, \quad \widetilde{f}\left(\widetilde{x}_{i}\right)=\widetilde{\theta}_{i}, \quad \widetilde{f}\left(\widetilde{y}_{i}\right)=-c \widetilde{\theta}_{i}^{*},\left.\quad \widetilde{f}\right|_{T_{n}}=\operatorname{id}_{T_{n}}
$$

of braided doubles in $\Gamma$ - Vect which covers the morphism $f: H_{0, c} \rightarrow \mathcal{H}_{Y_{n}}$, so that $\widetilde{H}_{0, c}$ is an extension of $H_{0, c}$ by the cocycle $[1, z] \in Z^{2}\left(S_{n}, C_{2}\right)$ covering $f$.

Proof. It follows from Proposition 10.5 and Lemma 10.6 that the map $\widetilde{f}$ is well-defined because the defining relations of $\widetilde{H}_{0, c}$ hold in $\left(\widetilde{\mathcal{H}_{Y_{n}}}\right)_{[1, z]}$. It remains to establish that $\widetilde{H}_{0, c}$ is a braided double in $\Gamma$ - Vect.

We use Proposition 7.14 where $V^{-}$is the free $\mathbb{C} \Gamma$-module with basis $\widetilde{x}_{1}, \ldots, \widetilde{x}_{n}$ and $V^{+}$is the free $\mathbb{C} \Gamma$-module with basis $\widetilde{y}_{1}, \ldots, \widetilde{y}_{n}$. Denote by $R^{-}$the subspace of $V^{-} \otimes_{\mathbb{C} \Gamma} V^{-}$spanned by the $z$-commutation relations (iii) and by $R^{+}$the subspace of $V^{+} \otimes_{\mathbb{C} \Gamma} V^{+}$spanned by the commutation relations (iv).

Crucially, because of the form of these quadratic relations we already know that the quadratic $\mathbb{C} \Gamma$-algebras $\left.T_{\mathbb{C} \Gamma}\left(V^{-}\right) /<R^{-}\right\rangle=(\mathbb{C} \Gamma)\left[\widetilde{x}_{1}, \ldots, \widetilde{x}_{n}\right]_{z}$ and $\left.T_{\mathbb{C} \Gamma}\left(V^{+}\right) /<R^{+}\right\rangle=(\mathbb{C} \Gamma)\left[\widetilde{y}_{1}, \ldots, \widetilde{y}_{n}\right]$ are free $\mathbb{C} \Gamma$-modules. It remains to check that $R^{+}$commutes with $V^{-}$in the free double $A_{\beta}(0,0)$, and similarly $V^{+}$commutes with $R^{-}$. We will check the latter, the former being analogous.

Definitely the commutator $\left[V^{+}, R^{-}\right]$lies in $V^{-} \otimes_{\mathbb{C} \Gamma} \mathbb{C} T_{n}$. Moreover, the map $\tilde{f}$ can be viewed as a map of $\mathbb{C} \Gamma$-algebras from $A_{\beta}(0,0)$ to $\left(\widetilde{\mathcal{H}_{Y_{n}}}\right)_{[1, z]}$. Because $\widetilde{f}\left(R^{-}\right)=0$ in $\left(\widetilde{\mathcal{H}_{Y_{n}}}\right)_{[1, z]}$ - recall that $\widetilde{\theta}_{1}, \ldots, \widetilde{\theta}_{n} z$-commute in $\left(\widetilde{H}_{Y_{n}}\right)_{[1, z]}$ - one trivially has $\widetilde{f}\left(\left[V^{+}, R^{-}\right]\right)=0$. So $\left[V^{+}, R^{-}\right]$is in $\operatorname{ker} \widetilde{f}$. But

$$
\left.\operatorname{ker} \widetilde{f}\right|_{V^{-}}=(1+z)\left(\widetilde{x}_{1}+\ldots+\widetilde{x}_{n}\right)
$$

because one can observe that $(1+z)\left(\widetilde{\theta}_{1}+\ldots+\widetilde{\theta}_{n}\right)=0$ is the only $\mathbb{C} \Gamma$-dependency between $\widetilde{\theta}_{1}, \ldots, \widetilde{\theta}_{n}$ in $Y_{n}$. Furthermore, ker $\left.\widetilde{f}\right|_{V^{-}} \otimes_{\mathbb{C} \Gamma} \mathbb{C} T_{n}=\left.\operatorname{ker} \widetilde{f}\right|_{V^{-}} \otimes_{\mathbb{C} \Gamma} \mathbb{C} T_{n}$. Therefore, $\left[V^{+}, R^{-}\right] \subset(1+z)\left(\widetilde{x}_{1}+\ldots+\right.$ $\left.\widetilde{x}_{n}\right) \otimes_{\mathbb{C} \Gamma} \mathbb{C} T_{n}$. It remains to observe that the substitution $z=1$ makes $\left[V^{+}, R^{-}\right]$zero - this is because the relations (i)-(viii) at $z=1$ become the relations in the rational Cherednik algebra $H_{0, c}$, which is a quadratic double in Vect. 
Remark 10.9. In the proof of the Theorem we observed that the $\widetilde{f}$-image of $\widetilde{H}_{0, c}$ in $\left(\widetilde{\mathcal{H}_{Y_{n}}}\right)_{[1, z]}$, although it automatically has triangular decomposition over $\mathbb{C} T_{n}$, is not a braided double in $\Gamma$ - Vect. Indeed, the submodule of $\left(\widetilde{Y_{n}}\right)_{[1, z]}$ generated by $\widetilde{f}\left(\widetilde{x}_{1}\right)=\widetilde{\theta}_{1}, \ldots, \widetilde{f}\left(\widetilde{x}_{n}\right)=\widetilde{\theta}_{n}$ fails to be free over $\mathbb{C} \Gamma$ : recall the relation $(1+z) \sum_{i=1}^{n} \widetilde{\theta}_{i}=0$. Consequently, the subalgebra

$$
\left(P_{n}\right)_{z}=\left\langle\widetilde{\theta}_{1}, \ldots, \widetilde{\theta}_{n}\right\rangle \subset \mathcal{B}\left(R X_{n}, q_{z}\right)
$$

is not a flat $C_{2}$-deformation of the coinvariant algebra $P_{n}$ of $S_{n}$. Observe that $\left.\left(P_{n}\right)_{z}\right|_{z=1}=P_{n}$ while $\left.\left(P_{n}\right)_{z}\right|_{z=-1}$ is Majid's algebra of flat connections, generated by $\alpha_{1}, \ldots, \alpha_{n}$ in $\mathcal{B}\left(X_{n}, q_{-1}\right)$. These two graded algebras differ already in degree 1 of the grading, because the Fomin-Kirillov elements $\theta_{1}, \ldots, \theta_{n}$ are linearly dependent (their sum is zero), unlike $\alpha_{1}, \ldots, \alpha_{n}$.

It would be interesting to describe a set of homogeneous defining relations for the $\mathbb{C C}_{2}$-algebra $\left(P_{n}\right)_{z}$; they would be a $z$-version of primitive symmetric functions:

Question 10.10. Describe the relations between the elements $\widetilde{\theta}_{1}, \ldots, \widetilde{\theta}_{n}$ in the algebra $\mathcal{B}\left(R X_{n}, q_{z}\right)$.

10.11. The spin Cherednik algebra. We finish the paper by observing that the covering Cherednik algebra $\widetilde{H}_{0, c}$ admits, as any braided double in $\Gamma$ - Vect, a specialisation at $z=-1$. By definition, this algebra will be a cocycle twist of $H_{0, c}$. The resulting algebra has triangular decomposition

$$
\left.\left(\widetilde{H}_{0, c}\right)\right|_{z=-1} \cong \mathbb{C}\left[x_{1}, \ldots, x_{n}\right]_{(-1)} \otimes\left(\mathbb{C} S_{n}\right)_{[1,-1]} \otimes \mathbb{C}\left[y_{1}, \ldots, y_{n}\right],
$$

where $\mathbb{C}\left[x_{1}, \ldots, x_{n}\right]_{(-1)}$ is the algebra generated by the pairwise anticommuting variables $x_{i}$, and $\left(\mathbb{C} S_{n}\right)_{[1,-1]}$ is the spin symmetric group as in 2.7 ,

The algebra $\left.\widetilde{H}_{0, c}\right|_{z=-1}$ coincides with the rational double affine Hecke algebra for the spin symmetric group, constructed using a different approach by Wang in 60. Moreover, Khongsap and Wang [36] proposed a covering version of this algebra, which is isomorphic to our $\widetilde{H}_{0, c}$.

Khongsap and Wang also gave versions of their construction for Weyl groups $W$ of types $B_{n}=C_{n}$ and $D_{n}$ in lieu of the symmetric group (the symmetric group $S_{n}$ is viewed as the Weyl group of type $\left.A_{n-1}\right)$. In our approach, it is natural to look for extensions of the rational Cherednik algebra of $W$ by a 2-cocycle on $W$. More generally, the technique should work for imprimitive complex reflection groups, denoted $W=G(m, p, n)$ in Shephard-Todd's classification; their Schur multipliers were computed by Read in [48]. We leave this generalisation to our upcoming paper [8].

\section{REFERENCES}

1. N. Andruskiewitsch, F. Fantino, G. A. García, and L. Vendramin, On Nichols algebras associated to simple racks, Groups, Algebras and Applications (César P. Milies, ed.), Contemporary Mathematics, vol. 537, American Mathematical Society, 2011, pp. 31-56. 
2. N. Andruskiewitsch and H.-J. Schneider, Finite quantum groups and Cartan matrices, Advances in Mathematics 154 (2000), no. 1, 1-45.

3. N. Anduskiewitsch and H.-J. Schneider, Pointed Hopf algebras, New directions in Hopf algebras (S. Montgomery and H.-J. Schneider, eds.), MSRI Publications, no. 43, Cambridge University Press, 2002, pp. 1-68.

4. P. Aschieri, F. Lizzi, and P. Vitale, Twisting all the way: From classical mechanics to quantum fields, Physical Review D 77 (2008), no. 2, $025037+$.

5. Y. Bazlov, Nichols-Woronowicz algebra model for Schubert calculus on Coxeter groups, Journal of Algebra 297 (2006), no. 2, 372-399.

6. Y. Bazlov and A. Berenstein, Braided doubles and rational Cherednik algebras, Advances in Mathematics 220 (2009), no. 5, 1466-1530.

7. _ Noncommutative Dunkl operators and braided Cherednik algebras, Selecta Mathematica, New Series 14 (2009), no. 3, 325-372.

8. _ Twisted Cherednik algebras (in preparation), 2013.

9. F. R. Beyl and J. Tappe, Group extensions, representations, and the Schur multiplicator, Springer-Verlag, 1982.

10. J. Bichon and G. Carnovale, Lazy cohomology: An analogue of the Schur multiplier for arbitrary Hopf algebras, Journal of Pure and Applied Algebra 204 (2006), no. 3, 627-665.

11. J. Bichon and C. Kassel, The lazy homology of a Hopf algebra, Journal of Algebra 323 (2010), no. 9, 2556-2590.

12. S. J. Brain and S. Majid, Quantisation of twistor theory by cocycle twist, Communications in Mathematical Physics 284 (2008), no. 3, 713-774.

13. A. Braverman and D. Gaitsgory, Poincaré-Birkhoff-Witt theorem for quadratic algebras of Koszul type, Journal of Algebra 181 (1996), no. 2, 315-328.

14. K. S. Brown, Cohomology of groups, Graduate Texts in Mathematics, vol. 87, Springer, 1982.

15. I. Cherednik, Double affine Hecke algebras, Cambridge University Press, 2005.

16. A. H. Clifford, Representations induced in an invariant subgroup, Annals of Mathematics (Second Series) 38 (1937), no. 3, 533-550.

17. J. H. Conway, R. T. Curtis, S. P. Norton, R. A. Parker, and R. A. Wilson, Atlas of finite groups: maximal subgroups and ordinary characters for simple groups, Clarendon Press; Oxford University Press, 1985.

18. J. W. Davies and A. O. Morris, The Schur multiplier of the generalized symmetric group, Journal of the London Mathematical Society (series 2) 8 (1974), 615-620.

19. P. Deligne and J. Milne, Tannakian categories (LaTeX version of the paper in Hodge Cycles, Motives, and Shimura Varieties, 1981, downloaded from http://www.jmilne.org/math), 2011.

20. V. G. Drinfel'd, Degenerate affine Hecke algebras and Yangians, Functional Analysis and Its Applications 20 (1986), no. 1, 58-60.

21. D. S. Dummit and R. M. Foote, Abstract algebra, 3 ed., Wiley, 2003.

22. S. Eilenberg and S. Mac Lane, Natural isomorphisms in group theory, Proc. Nat. Acad. Sci. U. S. A. 28 (1942), $537-543$.

23. P. Etingof, Exploring noncommutative algebras via deformation theory, preprint arXiv:math/0506144 4 (2009).

24. P. Etingof and V. Ginzburg, Symplectic reflection algebras, Calogero-Moser space, and deformed Harish-Chandra homomorphism, Inventiones Mathematicae 147 (2002), no. 2, 243-348.

25. P. Etingof and M. Graña, On rack cohomology, Journal of Pure and Applied Algebra 177 (2003), no. 1, 49-59.

26. S. Fomin and A. N. Kirillov, Quadratic algebras, Dunkl elements, and Schubert calculus, Advances in Geometry (J. L. Brylinski, ed.), Progr. Math., vol. 172, Birkhäuser Boston, 1997, pp. 147-182. 
27. T. Gateva-Ivanova and S. Majid, Quantum spaces associated to multipermutation solutions of level two, Algebras and Representation Theory 14 (2011), no. 2, 341-376.

28. M. Gerstenhaber, On the deformation of rings and algebras, Annals of Mathematics 79 (1964), no. 1, 59-103.

29. I. Gordon, Baby Verma modules for rational Cherednik algebras, Bulletin of the London Mathematical Society 35 (2003), no. 3, 321-336.

30. M. Graña, I. Heckenberger, and L. Vendramin, Nichols algebras of group type with many quadratic relations, Advances in Mathematics 227 (2011), no. 5, 1956-1989.

31. J. A. Green, On the number of automorphisms of a finite group, Proceedings of the Royal Society of London. Series A, Mathematical and Physical Sciences 237 (1956), no. 1211, 574-581.

32. R. L. Griess, Schur multipliers of the known finite simple groups. III, Proceedings of the Rutgers group theory year, 1983-1984 (New Brunswick, N.J., 1983-1984), Cambridge University Press, 1985, pp. 69-80.

33. P. Guillot and C. Kassel, Cohomology of invariant drinfeld twists on group algebras, International Mathematics Research Notices 2010, no. 10, 1894-1939.

34. P. Hall, The classification of prime-power groups, Journal für die reine und angewandte Mathematik 182 (1940), $130-141$.

35. A. Joyal and R. Street, Braided tensor categories, Advances in Mathematics 102 (1993), no. 1, 20-78.

36. T. Khongsap and W. Wang, Hecke-Clifford algebras and spin Hecke algebras, II: the rational double affine type, Pacific Journal of Mathematics 238 (2008), no. 1, 73-103.

37. G. Lusztig, Introduction to quantum groups, corrected ed., Birkhäuser Boston, 1993.

38. S. Mac Lane, Categories for the working mathematician, 2nd ed., Graduate Texts in Mathematics, Springer, 1998.

39. S. Majid, Free braided differential calculus, braided binomial theorem, and the braided exponential map, Journal of Mathematical Physics 34 (1993), no. 10, 4843-4856.

40. Double-bosonization of braided groups and the construction of $U_{q}(\mathfrak{g})$, Mathematical Proceedings of the Cambridge Philosophical Society 125 (1999), no. 1, 151-192.

41. _ Foundations of quantum group theory, Cambridge University Press, 2000.

42. __ A quantum groups primer, London Mathematical Society Lecture Note Series (No. 292), Cambridge University Press, 2002.

43. _ Noncommutative differentials and Yang-Mills on permutation groups $S_{n}$, Hopf algebras in noncommutative geometry and physics, Lecture Notes in Pure and Appl. Math., no. 239, Dekker, NY, 2005, pp. 189-213.

44. S. Majid and R. Oeckl, Twisting of quantum differentials and the Planck scale Hopf algebra, Communications in Mathematical Physics 205 (1999), no. 3, 617-655.

45. A. Milinski and H.-J. Schneider, Pointed indecomposable Hopf algebras over Coxeter groups, New trends in Hopf algebra theory (La Falda, 1999) (N. Andruskiewitsch, W. R. Ferrer Santos, and H.-J. Schneider, eds.), Contemporary Mathematics, no. 267, American Mathematical Society, 2000, pp. 215-236.

46. W. D. Nichols, Bialgebras of type one, Communications in Algebra 6 (1978), no. 15, 1521-1552.

47. F. Panaite, M. D. Staic, F. Van Oystaeyen, Pseudosymmetric braidings, twines and twisted algebras, Journal of Pure and Applied Algebra 214 (2010), no. 6, 867-884.

48. E. W. Read, On the Schur multipliers of the finite imprimitive unitary reflection groups, Journal of the London Mathematical Society (series 2) $\mathbf{1 3}$ (1976), no. 1, 150-154.

49. J. J. Rotman, An introduction to the theory of groups, Springer-Verlag, 1994.

50. A. Savage, Braided and coboundary monoidal categories, preprint arXiv:0804.4688v2 (2008). 
51. P. Schauenburg, Hopf bimodules, coquasibialgebras, and an exact sequence of Kac, Advances in Mathematics 165 (2002), no. 2, 194-263.

52. I. Schur, Über die Darstellung der endlichen Gruppen durch gebrochene lineare Substitutionen, Journal für die reine und angewandte Mathematik 127 (1904), 20-50.

53. — Untersuchungen über die Darstellung der endlichen Gruppen durch gebrochene lineare Substitutionen, Journal für die reine und angewandte Mathematik 132 (1907).

54. _ _ Über die Darstellung der symmetrischenund der alternierenden Gruppen durch gebrochene lineare Substitutionen, Journal für die reine und angewandte Mathematik 139 (1911), 155-250.

55. J. P. Serre, Complex semisimple Lie algebras, Springer, 2001.

56. M. E. Sweedler, Hopf algebras, Mathematics Lecture Note Series, W. A. Benjamin, Inc., New York, 1969.

57. C. Vafa and E. Witten, On orbifolds with discrete torsion, Journal of Geometry and Physics 15 (1995), no. 3, $189-214$.

58. L. Vendramin, Fomin-Kirillov algebras, preprint arXiv:1210.5423 (2012).

59. N Nichols algebras associated to the transpositions of the symmetric group are twist-equivalent, Proceedings of the American Mathematical Society 140 (2012), no. 11, 3715-3723.

60. W. Wang, Double affine Hecke algebras for the spin symmetric group, Mathematics Research Letters 16 (2009), $1071-1085$

61. S. L. Woronowicz, Differential calculus on compact matrix pseudogroups (quantum groups), Communications in Mathematical Physics 122 (1989), no. 1, 125-170.

School of Mathematics, University of Manchester, Oxford Road, Manchester, M13 9PL, UK

E-mail address: yuri.bazlov@manchester.ac.uk

Department of Mathematics, University of Oregon, Eugene, OR 97403, USA

E-mail address: arkadiy@math.uoregon.edu 